\title{
Distribution and Status of Bird, Small Mammal, Reptile, and Amphibian Species, South Dakota Field Office-BLM
}

\author{
Prepared for: \\ Bureau of Land Management \\ South Dakota Field Office \\ Prepared by: \\ Daniel A. Bachen and Bryce A. Maxell \\ Montana Natural Heritage Program \\ a cooperative program of the \\ Montana State Library and the University of Montana
}

November 2014

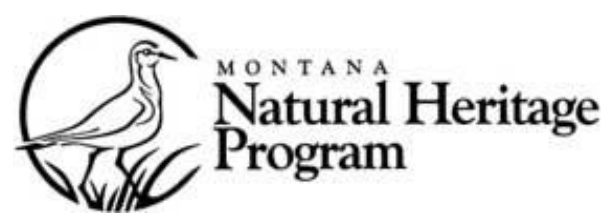





\title{
Distribution and Status of Bird, Small Mammal, Reptile, and Amphibian Species, South Dakota Field Office-BLM
}

\author{
Prepared for: \\ Bureau of Land Management \\ South Dakota Field Office
}

Agreement Number:

L13AC00190

Prepared by:

Daniel A. Bachen and Bryce A. Maxell
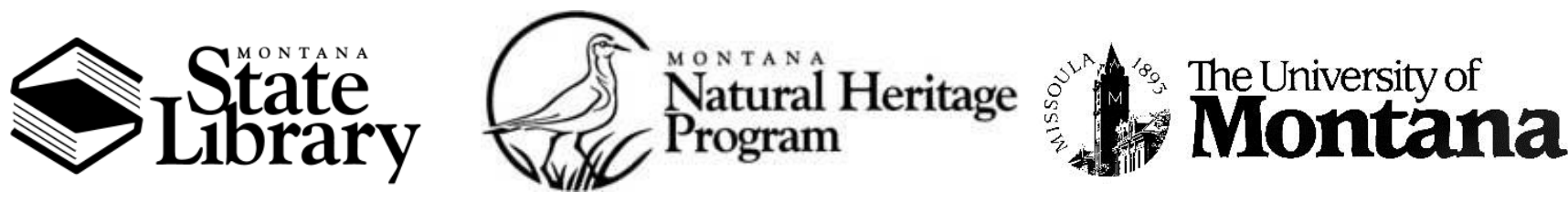

(c) 2014 Montana Natural Heritage Program

P.O. Box 201800 • 1515 East Sixth Avenue • Helena, MT 59620-1800 • 406-444-3290 
This document should be cited as follows:

Bachen, D. A, and B. A. Maxell. 2014. Distribution and status of bird, small mammal, reptile, and amphibian species, South Dakota Field Office-BLM. Report to the Bureau of Land Management, South Dakota Field Office. Montana Natural Heritage Program, Helena, Montana 25 pp. plus appendices. 


\section{EXECUTIVE SUMMARY}

To date, federal lands administered by the Bureau of Land Management (BLM) in South Dakota have received limited attention for baseline inventories of nongame species including bird, terrestrial small mammal, bat, amphibian and reptile species. Documenting baselines for distribution, habitat association, and relative status of these species, particularly species listed as Sensitive by the BLM or rare, threatened, or endangered by the state, can aid in conservation of these species. To address this need we conducted structured surveys to document these species within BLM administered lands in western South Dakota, using point count surveys for birds, trap arrays for small mammals, passive acoustic bat detectors for bats, night time calling surveys for amphibians, and visual encounter surveys of wetlands for amphibians and reptiles.

Using structured surveys over the spring and summer of 2014, we documented 100 bird species, including 13 listed as Sensitive by the BLM, 15 terrestrial small mammal species, 6 bat species, and 5 amphibian species, including 2 Sensitive species. While conducting this work we also incidentally recorded 1,260 additional animal observations, including an additional 25 bird species, 17 mammal species, and 8 reptile species. While conducting surveys for birds we documented several BLM Sensitive species of note, including the Sprague's Pipit (Anthus spragueii), Blue-Gray Gnatcatcher (Polioptila caerulea), Bald Eagle (Haliaeetus leucocephalus), and Baird's Sparrow (Ammodramus bairdii). Although we did not capture any Sensitive small mammal species, we did capture 2 species of shrew that represent significant records for the state. In central South Dakota we captured a Least Shrew (Cryptotis parva), which not only was the first record of the species in Stanley County, but also is one of the northern most observations of this specie in the state (Backlund 2002). In Northwest South Dakota we captured a shrew in sagebrush steppe that is likely a range extension for Montane Shrew (Sorex monticolus) or Dwarf Shrew (S. nanus), but are still working on definitive identification of this specimen. Although we documented several amphibian and reptile species listed as Sensitive, we documented one particularly rare species. In a small area of badlands in Western Butte County we incidentally encountered a Greater Short-horned Lizard (Phrynosoma hernandesi). In this area we also documented other rare species like the Sage Thrasher (Oreoscoptes montanus) and Brewer's Sparrow (Spizella breweri).

For birds, we found the highest species diversity in Central South Dakota, where the prairie transitions to conifer forests, and the lowest species diversity within the conifer forests of the Black Hills, although this low diversity may reflect inclement weather conditions at the time of the survey. For small terrestrial mammals, we found the greatest species diversity in the conifer forests of the Black Hills and the lowest species diversity in the mixedgrass prairies of west central South Dakota. For bats, we found a cessation of activity at all five of our prairie dominated monitoring sites between December 2013 and March 2014. For amphibians, we detected the highest diversity of species in the mixedgrass prairies of central South Dakota in close proximity to the Belle Fourche River and the 
lowest species diversity in mixedgrass prairie in west central South Dakota.

Overall, we found riparian woodland habitats to be extremely limited on BLM lands within the South Dakota BLM Field Office with some evidence of lack of regeneration of Plains Cottonwoods. We encourage management focus on this habitat to conserve existing riparian woodlands and restore riparian woodland areas that are becoming deforested by promoting management regimes that mimic the natural frequency and intensity of disturbances resulting from historical grazing, fire, and flood regimes. More broadly, management regimes that mimic the natural frequency and intensity of disturbances to all major habitat cover types should be encouraged across the South Dakota BLM Field Office.

In order to make survey and detection information from this and other surveys more readily available for resource management plans and project-level planning, we have made it available online through the Montana Natural Heritage Program's MapViewer

http://mtnhp.org/mapviewer/ 


\section{ACKNOWLEDGEMENTS}

We extend considerable thanks and

appreciation to Rebecca Newton, wildlife biologist with the BLM South Dakota Field

Office, for recognizing the importance of this project and shepherding the project through

BLM channels. Thanks to Scott Blum, Braden

Burkholder, and Shannon Hilty for analyzing bat calls, Scott Blum for appending the data to the
Montana Natural Heritage Program's central animal observation database, and Paul Hendricks for originally proposing the project. Also we acknowledge and thank the South Dakota Game and Fish for allowing us to conduct small mammal trapping and amphibian and reptile surveys within the state.

This project was supported by an agreement between the Bureau of Land Management and the Montana Natural Heritage Program, a cooperative program of the Montana State Library and the University of Montana (BLM L13AC00190) 


\section{TABLE OF CONTENTS}

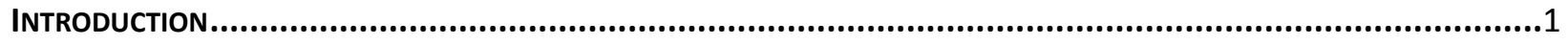

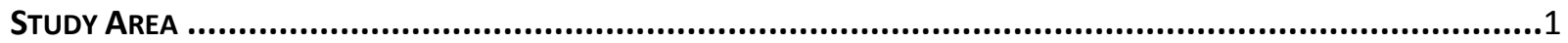

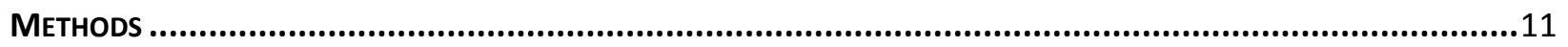

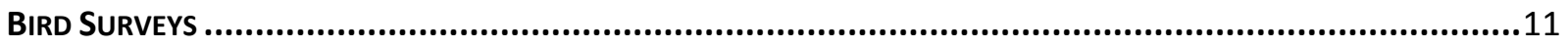

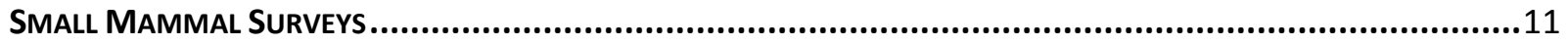

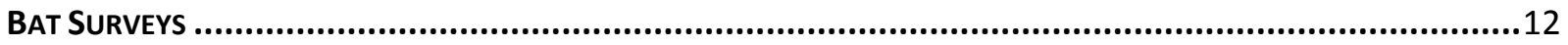

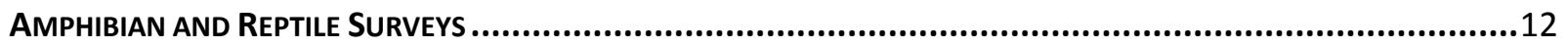

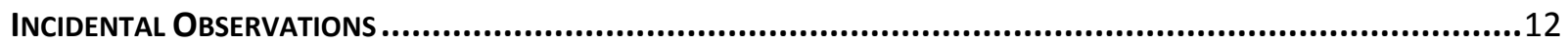

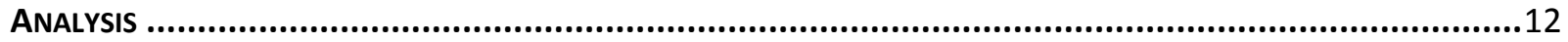

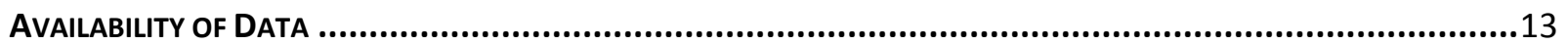

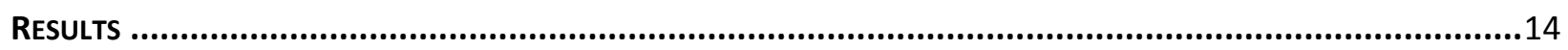

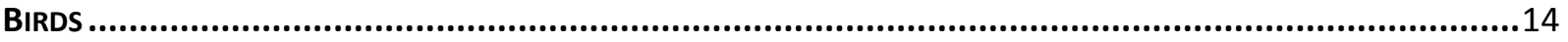

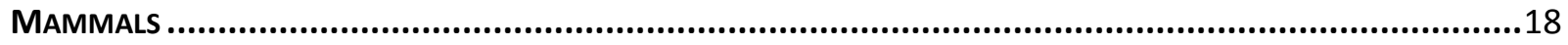

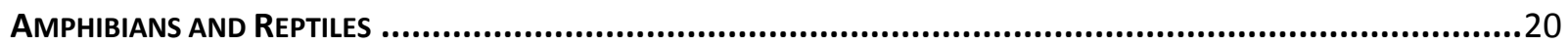

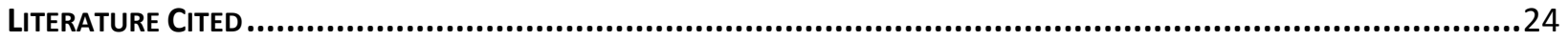

\section{LIST OF FIGURES}

Figure 1. Primary survey areas in western South Dakota .................................................................... 2

Figure 2. Butte County primary area survey locations …..................................................................... 3

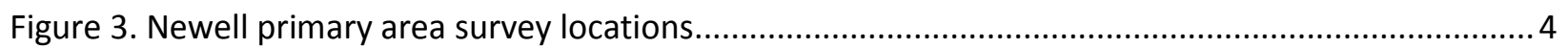

Figure 4. Fort Meade primary area survey locations .............................................................................. 5

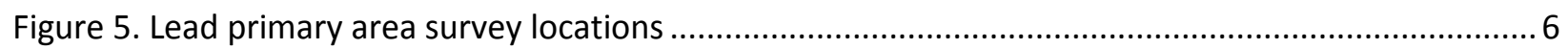

Figure 6. Southern Black Hills primary area survey locations ............................................................... 7

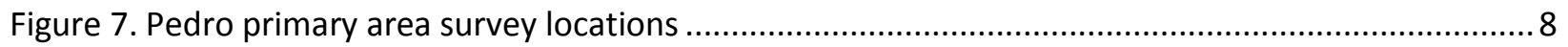

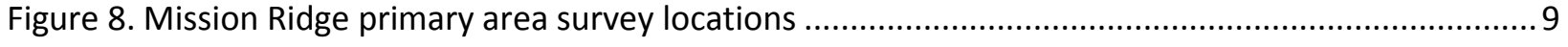

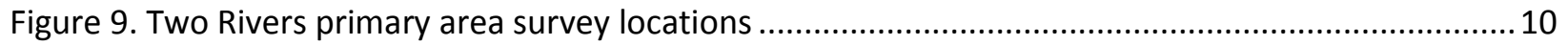

\section{LIST OF TABLES}

Table 1. Bird surveys: effort.

Table 2. Bird species: number of points detected, incidental observations, proportion of primary areas detected, global and state ranks ............................................... 15

Table 3. Mammal surveys: effort

Table 4. Mammal species: number of points detected, incidental observations, proportion of primary areas detected, global and state ranks. 


\section{TABLE OF CONTENTS (CONT.)}

Table 5. Amphibian calling surveys: effort

Table 6. Amphibian and reptile species: number of points detected, incidental observations, proportion of primary areas detected, global and state ranks

\section{APPENDICES}

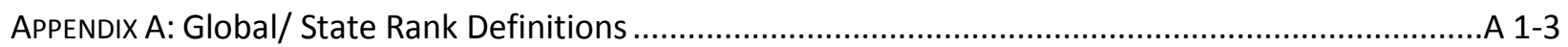

APPENDIX B: Echolocation Call Characteristics of Montana Bats ....................................................... 1 1-6

APPENDIX C: Baseline Status Indices of Detected Species: Bird Surveys ............................................ 1-13

APPENDIX D: Baseline Status Indices of Detected Species: Small Mammal Surveys ............................. D 1-8

APPENDIX E: Baseline Status Indices of Detected Species: Bat Surveys.................................................. E 1-3

APPENDIX F: Baseline Status Indices of Detected Species: Amphibian Calling Surveys.......................... F 1-2 


\section{INTRODUCTION}

Federal lands administered by the South Dakota Field Office of the Bureau of Land Management (BLM), have received limited attention for baseline inventories of birds, small terrestrial mammals, bats, amphibians, or aquatic reptiles. Current observation data in the Montana Natural Heritage Program (MNHP) database for these lands are limited to a few bird point counts, miscellaneous small mammal surveys and incidental observations. Thus, there is a need for structured surveys to provide baseline information on the distribution and status of these taxa, especially those with state or global conservation status ranks of S1-S3 or G1-G3 (Appendix A) and those listed as Sensitive by the BLM. This information is vital to broadscale resource management plans and projectlevel decisions.

To address this need we conducted structured surveys to document these species within BLM administered lands in western South Dakota, using point count surveys for birds, trap arrays for small mammals, acoustic bat detectors for bat, night time calling surveys for amphibians, and visual encounter surveys of wetlands for amphibians and reptiles. In addition to increasing the data within the MNPH database, conducting surveys to document the distribution, habitat association, and relative status of species across state BLM lands will provide managers with valuable information that can be used to manage lands for the benefit of wildlife and the people that work and recreate on these lands. Documentation of species, particularly species that are listed as sensitive by the BLM can help guide land management planning and practices to facilitate conservation of these species.

\section{STUdy AREA}

Across western South Dakota, the BLM manages lands in dispersed blocks of varying size. These lands are characterized by a diversity of soil types, elevations, geographic features, and vegetation cover. Except in the Black Hills, soils throughout the study area are predominantly clays and sandy loams. In the Black Hills soils are primarily rocky and silty loams, transitioning to sandy and clay loams in the foothills (NRCS 2014). Elevations range from approximately $500 \mathrm{~m}$ near the Missouri river in the east, to approximately $1,750 \mathrm{~m}$ in the Black Hills to the west.

Lands in the north of the state are dominated primarily by mixedgrass prairie with sparse low shrub cover and occasional badlands. Lands in the Black Hills are dominated by conifer forests with occasional stands of aspen and open meadows surrounding woody riparian areas dominated by willows. At the southern end of the Black Hills the vegetation transitions to patchy ponderosa pine and juniper forests, interspersed with large areas of mixedgrass prairie. In the eastern foot hills of the Black Hills, conifer forests are interspersed with deciduous forests dominated by burr oak and there is a transition to mixedgrass prairie. In the central portion of western South Dakota the vegetation is primarily mixedgrass prairie in the uplands transitioning to juniper and ponderosa pine forests, and badlands in the river breaks, with deciduous forests dominated by cottonwoods in the river bottoms. In the east along the Missouri river, the uplands are primarily dominated by mixedgrass prairie and large areas have been converted to agriculture. The river breaks in this area are primarily 
dominated by prairie, or badlands with sparse shrubland. Precipitation in all areas is greatest in the summer due to numerous thunderstorms. Within the study area, the Black Hills receive the most moisture, on average $76-$ $89 \mathrm{~cm}$ per year. The remainder of the area receives $25-38 \mathrm{~cm}$ of annual precipitation (NOAA 2012).

Based on these differences in habitat and geography we selected representative sampling areas, to ensure adequate documentation of wildlife species across BLM managed lands as these features can influence species distribution (Heisler et al. 2013). We selected these primary sampling areas based on 2 criteria: first that there was a relatively large block or blocks of BLM managed land in the area $\left(>3 \mathrm{~km}^{2}\right)$ with road access, and second that these lands were representative of the surrounding habitat and unique from the other primary areas. Based on these criteria we selected 8 areas within the state (Figure 1).

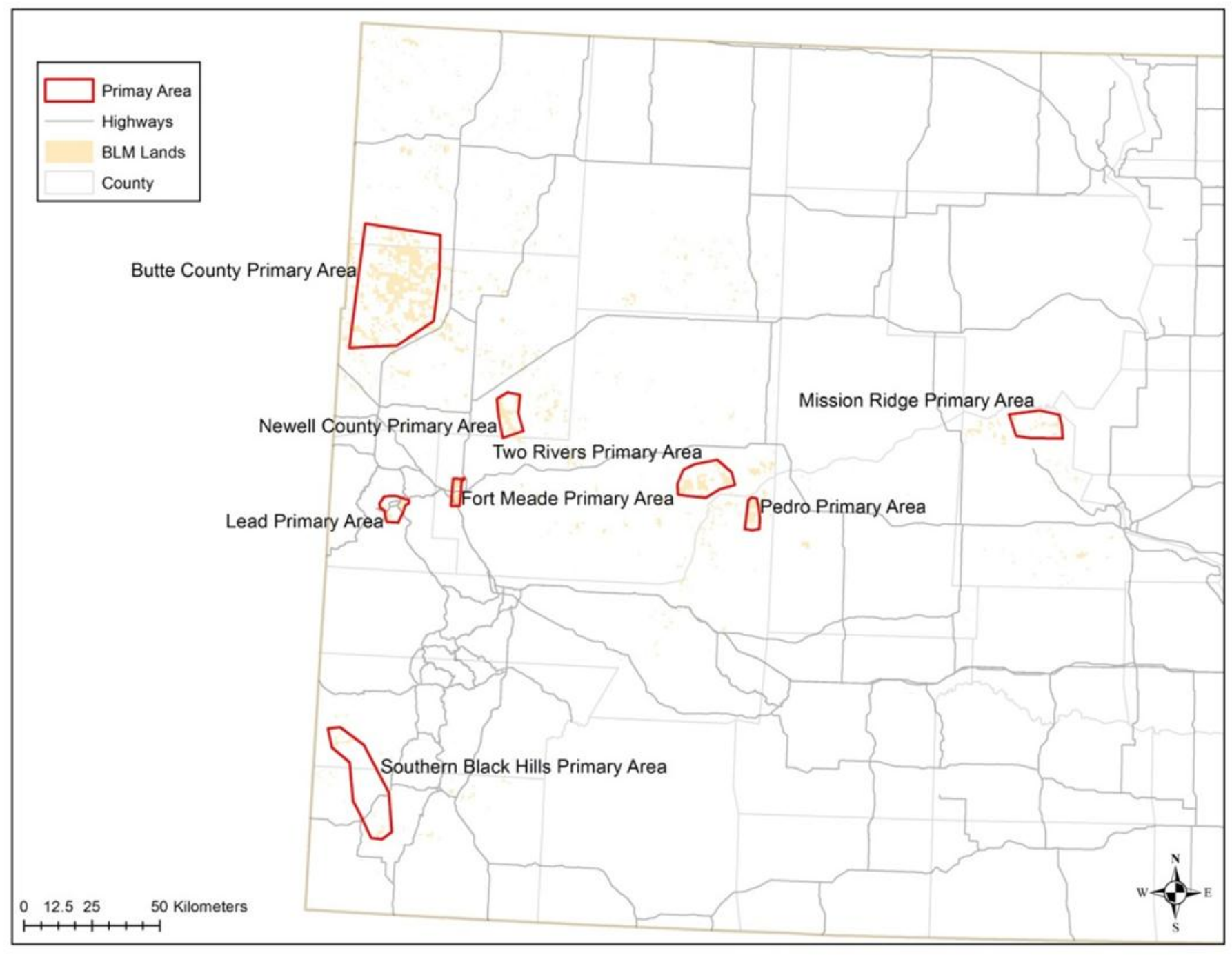

Figure 1. Primary survey areas in western South Dakota 


\section{Butte County Primary Survey Area}

This area is in northwestern Butte County and the southern portion of Harding County (hereafter referred to as Butte County primary area) (Figure 2). It is approximately $1,230 \mathrm{~km}^{2}$ in size and is characterized by rolling hills with ephemeral creeks and numerous stock reservoirs. The primary vegetation communities are mixedgrass prairie with sparse sagebrush steppe in the uplands and cotton wood and green ash stands along some riparian areas. The soils here are primarily clays.

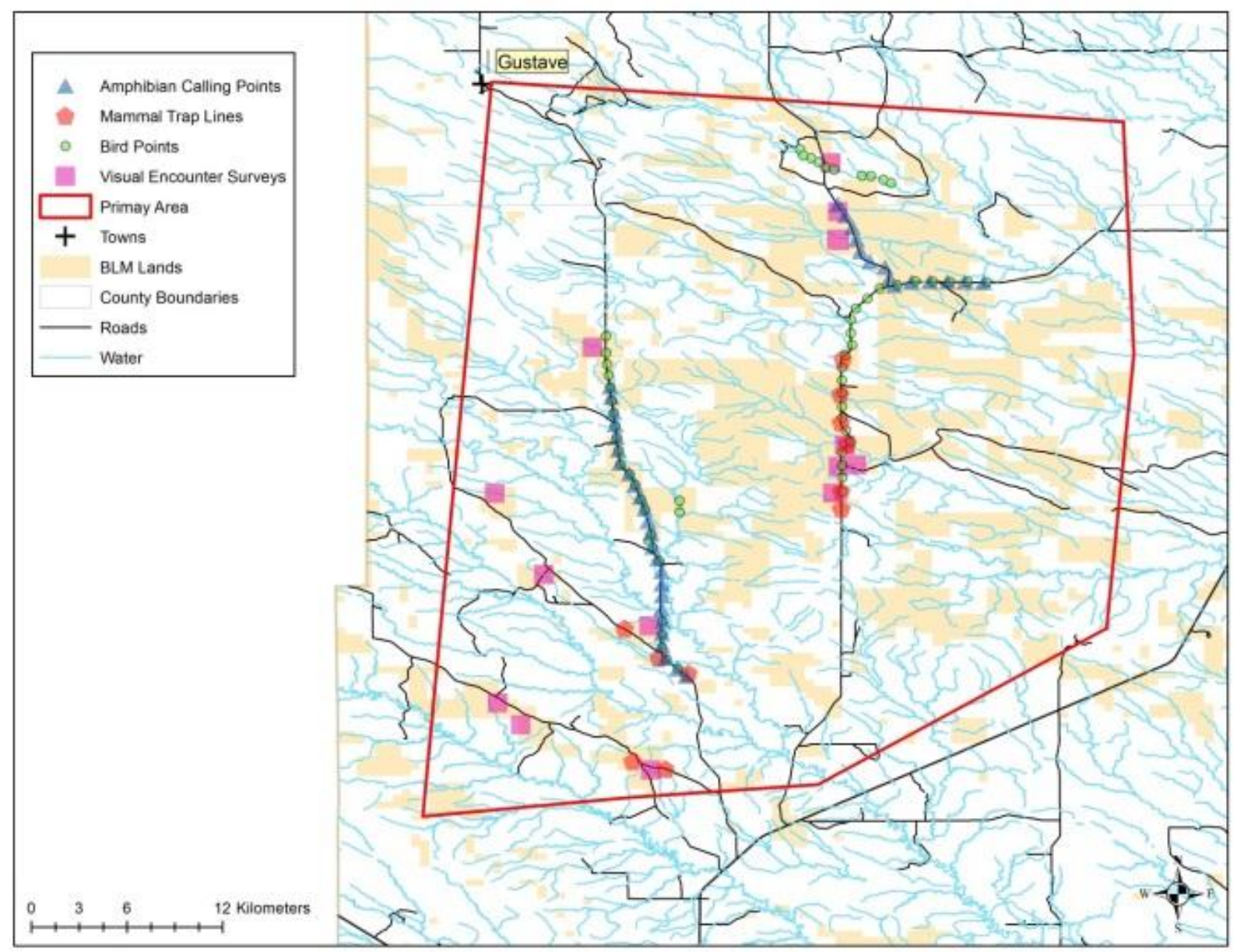

Figure 2.Butte County primary area survey locations 


\section{Newell Primary Survey Area}

This area is east of Newell in southeastern Butte County (Figure 3). It is approximately $120 \mathrm{~km}^{2}$ in size, and characterized by rolling hills, plains, and breaks dominated by mixed grass prairie, with occasional stock reservoirs. The soils here are primarily clays.

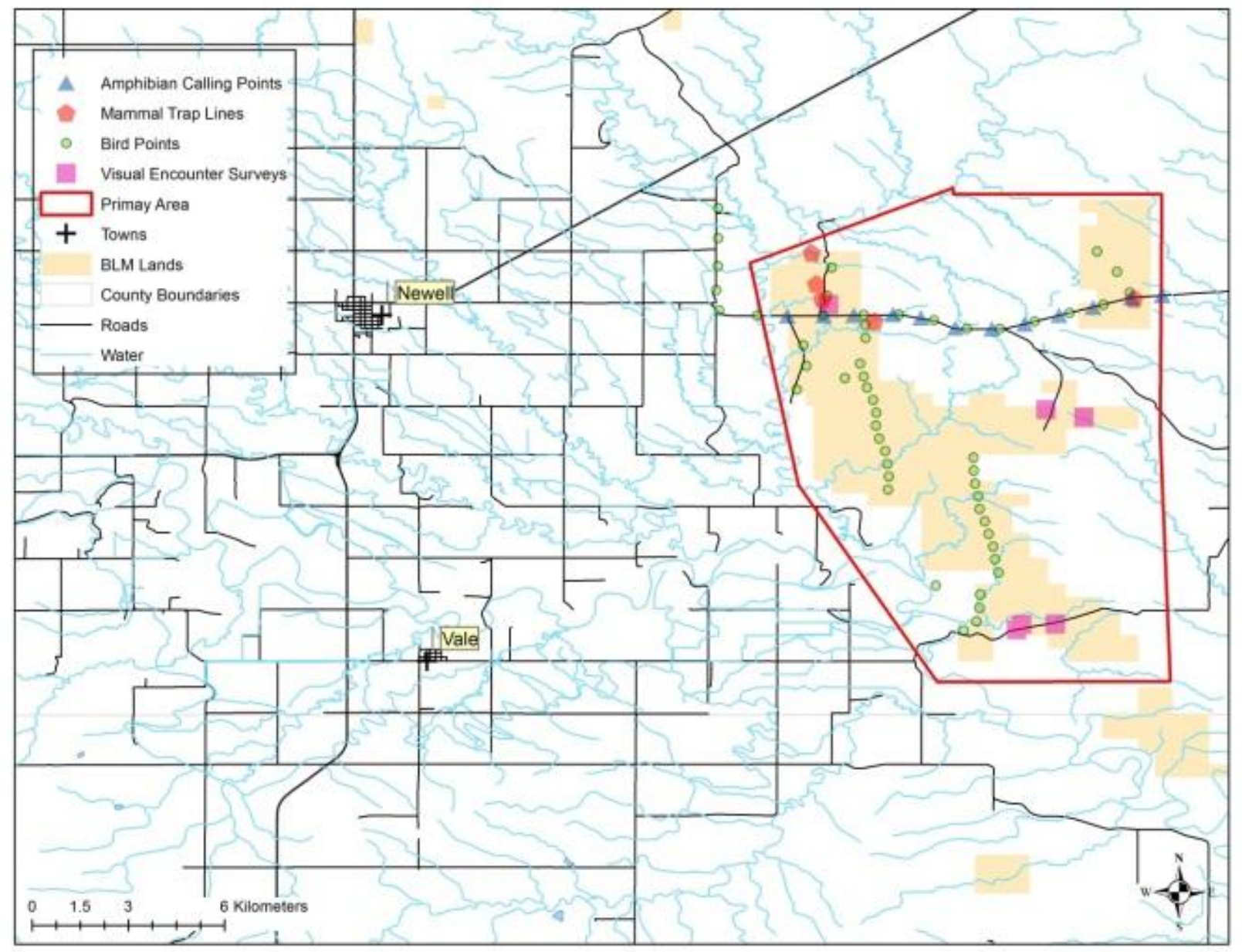

Figure 3.Newell primary area survey locations 


\section{Fort Meade Primary Survey Area}

This area is outside of Sturgis in western Meade County (Figure 4). It is relatively small, approximately 30 $\mathrm{km}^{2}$ in size, and is located at the transition of the Black Hills to the eastern prairie. The northern portion of this area is dominated by mixedgrass prairie, with occasional reservoirs and small streams, and bur oak stands. The central and southern portions of this area are rugged with numerous small mountains and sandstone breaks. There are occasional areas of mixedgrass prairie, but this portion of the area is dominated by ponderosa pine forests with burr oak and ash stands along small creeks and cotton wood forests along larger waterways. The soils here are primarily clay loams and sandy loams.

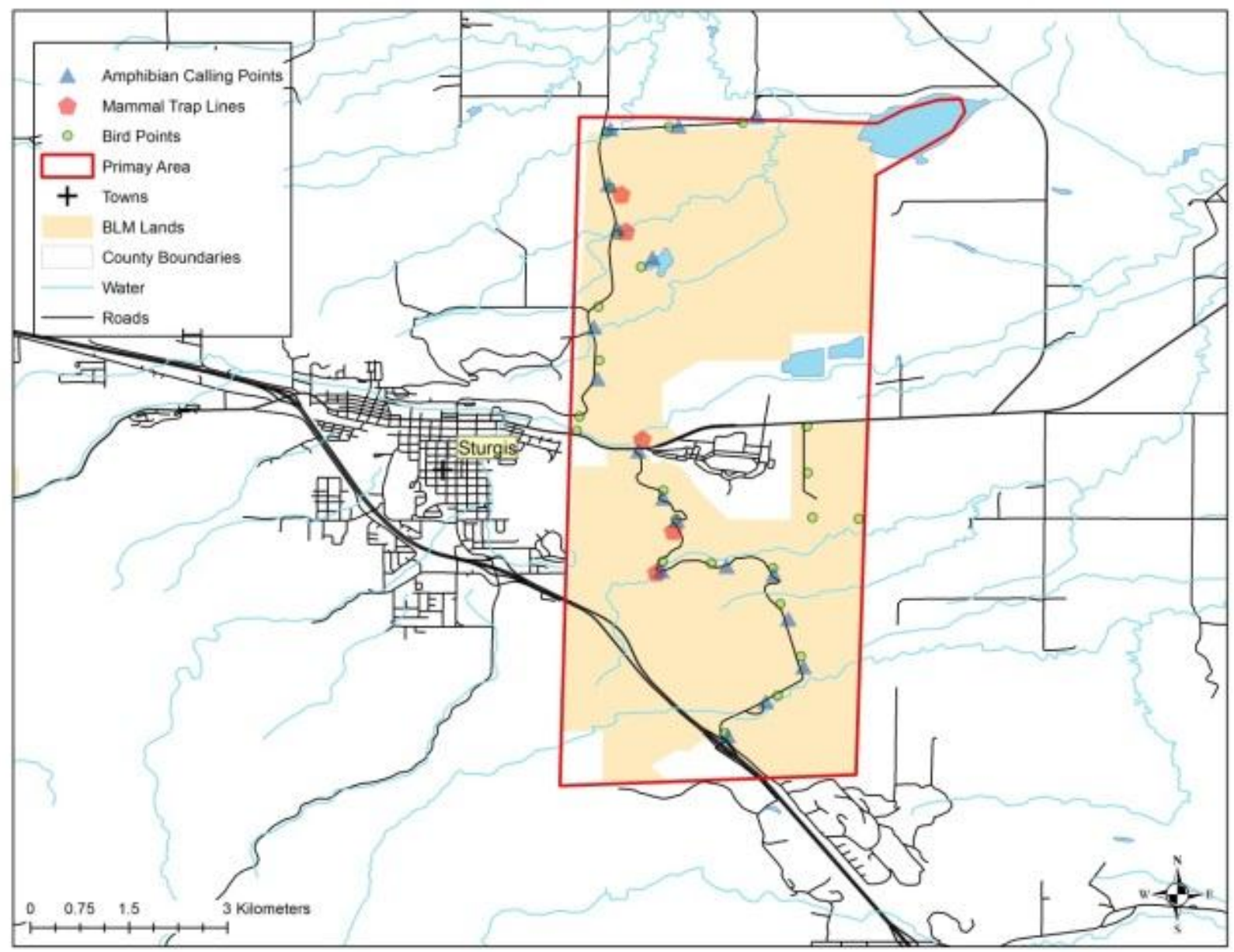

Figure 4. Fort Meade primary area survey locations 


\section{Lead Primary Survey Area}

This area is in the north central Black Hills in central Lawrence County near the towns of Lead and Deadwood (Figure 5). It is approximately $70 \mathrm{~km}^{2}$ in size and is rugged and dominated by conifer forest with occasional stands of aspen. Along waterways there are wet meadows dominated by brome grasses and willows. The soils of this area are primarily rocky and loamy.

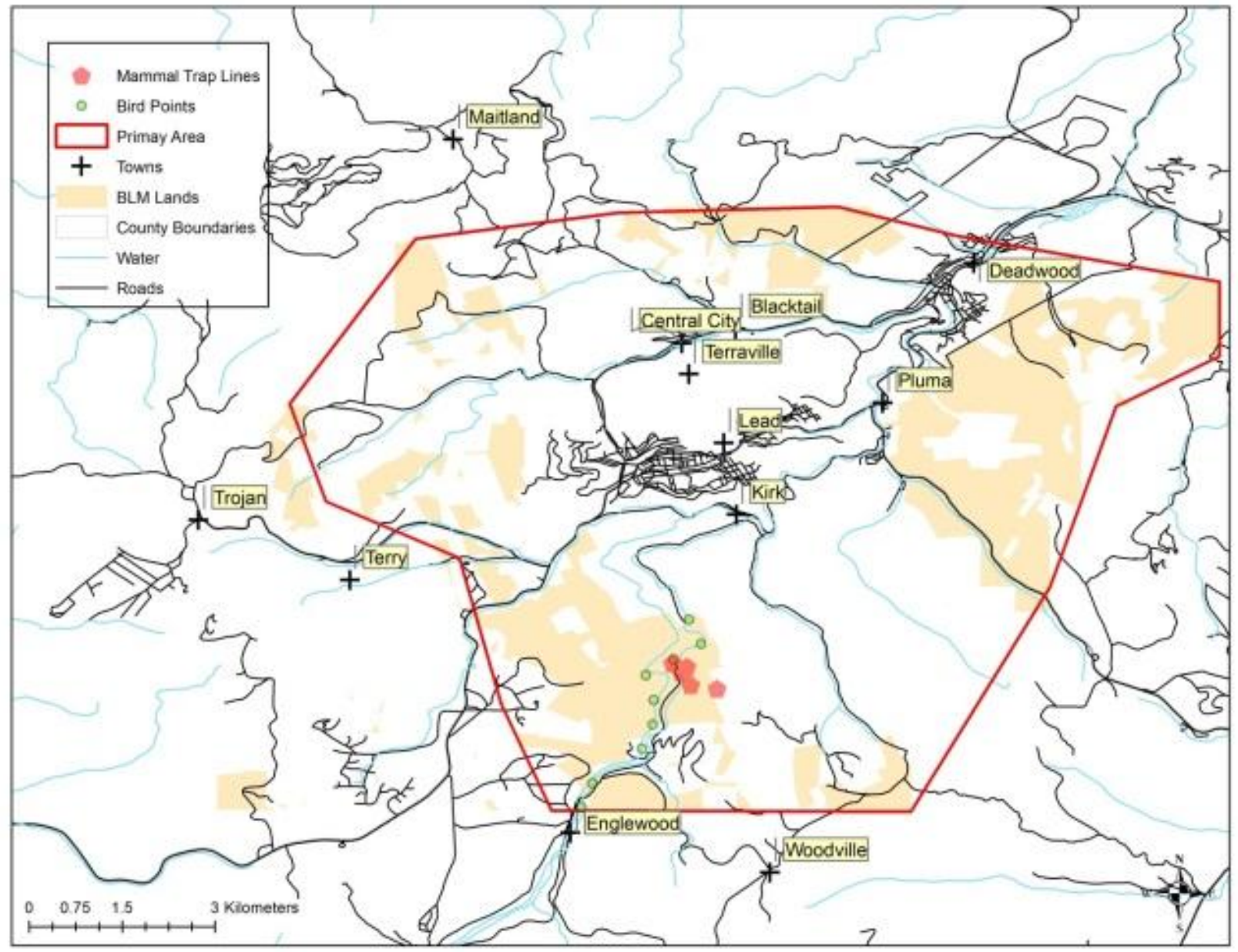

Figure 5. Lead primary area survey locations 
Southern Black Hills Primary Survey Area

This area is located in the southern Black Hills west of Hot Springs in southern Custer and northern Fall River Counties (Figure 6). It had two areas of BLM managed lands: one $8 \mathrm{~km}^{2}$ block located in southeastern Custer County; and the Fossil Cycad National Monument, is located off Highway 18 between Hot Springs and Edgemont in Northern Fall River County. This area has rolling hills with occasional deep ravines, dominated by either ponderosa pine forest or mixedgrass prairie. The soils of this area are rocky, with areas of silty and sandy loams and exposed sandstone bedrock.

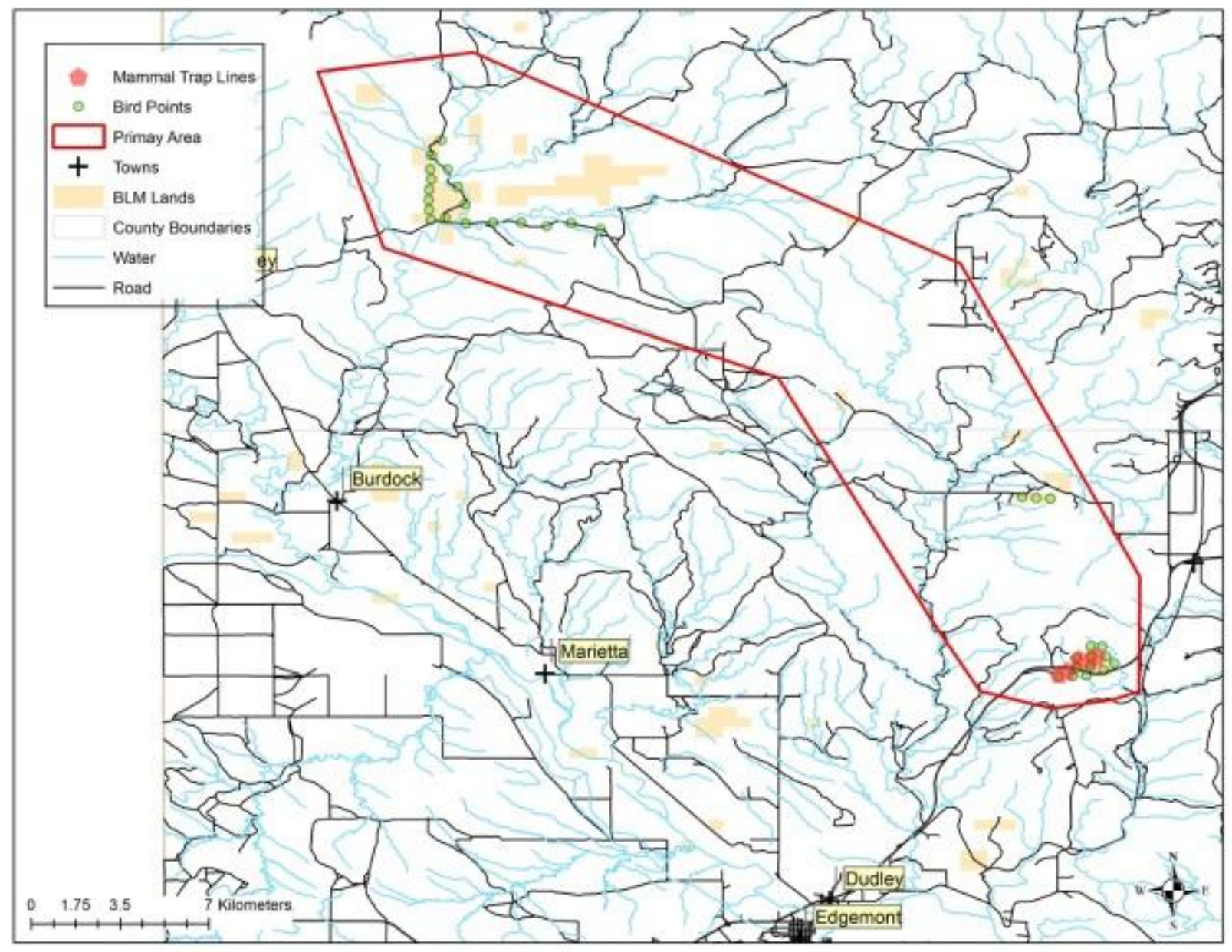

Figure 6. Southern Black Hills primary area survey locations 


\section{Pedro Primary Survey Area}

This area is located in the northern Hakkon County in the vicinity of an abandoned town, Pedro (Figure 7). It is approximately $34 \mathrm{~km}^{2}$ in size and is located in the breaks south of the Belle Fourche River.

Vegetation is primarily juniper forests on east and north facing slopes, shrublands dominated by yucca and sumac and mixed grass prairie on the drier western facing slopes. The soils here are primarily clays.

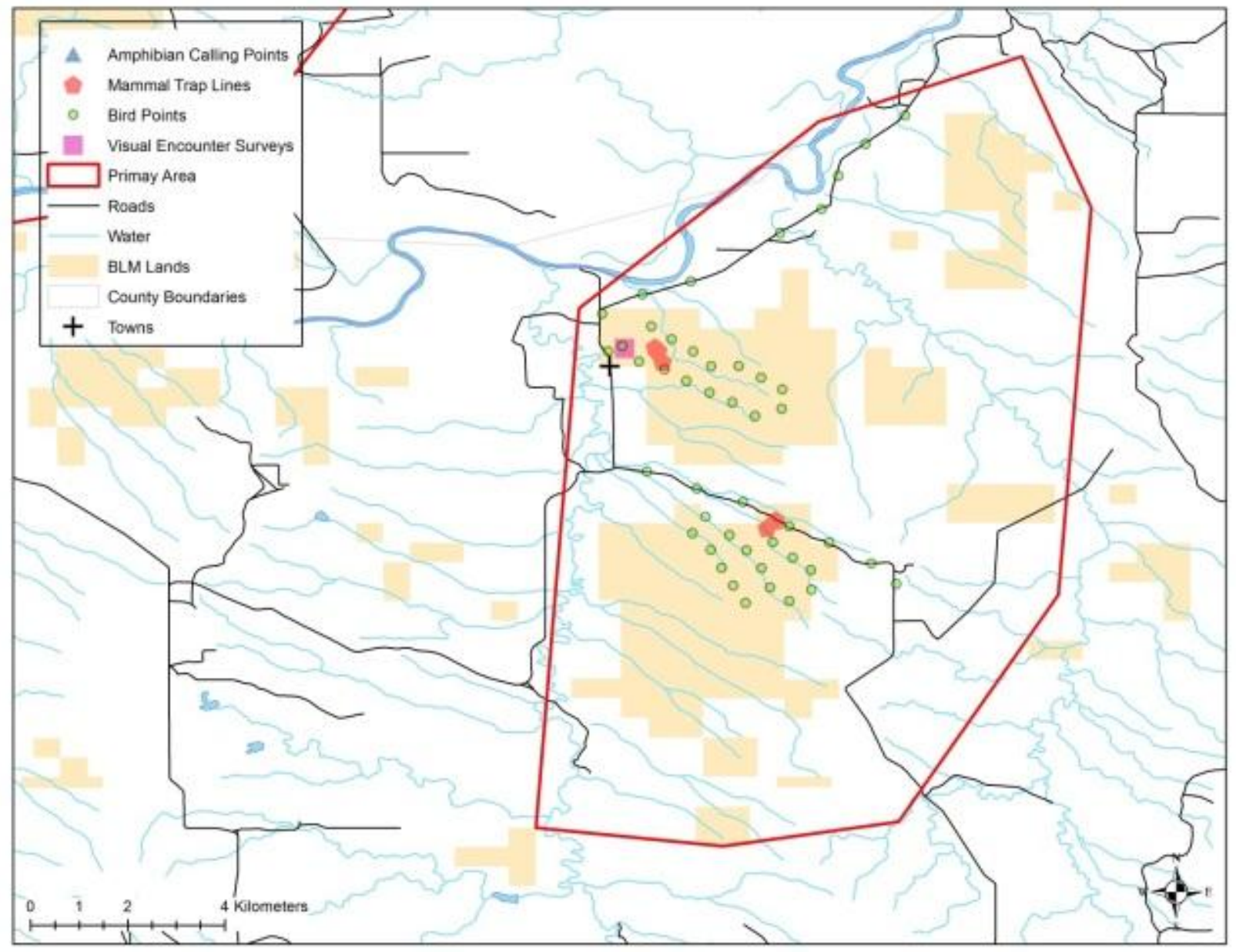

Figure 7. Pedro primary area survey locations 


\section{Mission Ridge Primary Survey Area}

This area is located northwest of Pierre in the vicinity of the town of Mission Ridge south of Lake Oahe in northern Stanley County (Figure 8). Although this area was relatively large, approximately $190 \mathrm{~km}^{2}$ in size, the BLM managed lands within it are dispersed small blocks. The area is characterized by rolling uplands covered by mixedgrass prairie with badland breaks along the edge of the reservoir. The soils here are primarily clays with areas of sandy loams.

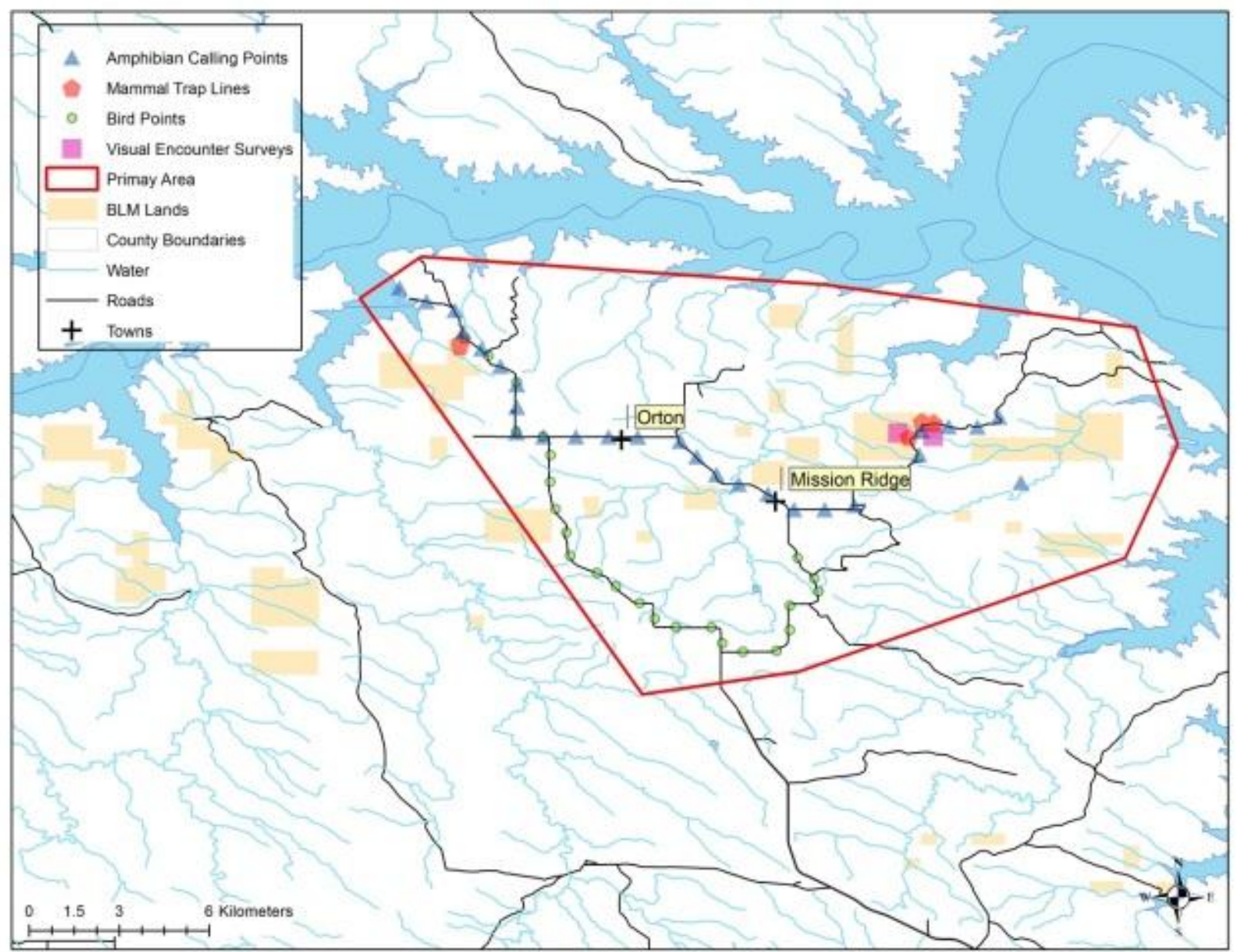

Figure 8. Mission Ridge primary area survey locations 
Two Rivers Primary Survey Area

This area is located in the river breaks north of the confluence of the Belle Fourche and Cheyenne Rivers in southern Ziebach County (Figure 9). It is approximately $150 \mathrm{~km}^{2}$ in size and consists of 3 blocks of BLM managed land. The uplands are dominated by mixedgrass prairie, while the breaks have sparse ponderosa pine forest, small outcrops of sandstone, badlands, and juniper forests in the draws. The soils are primarily clays with areas of loam.

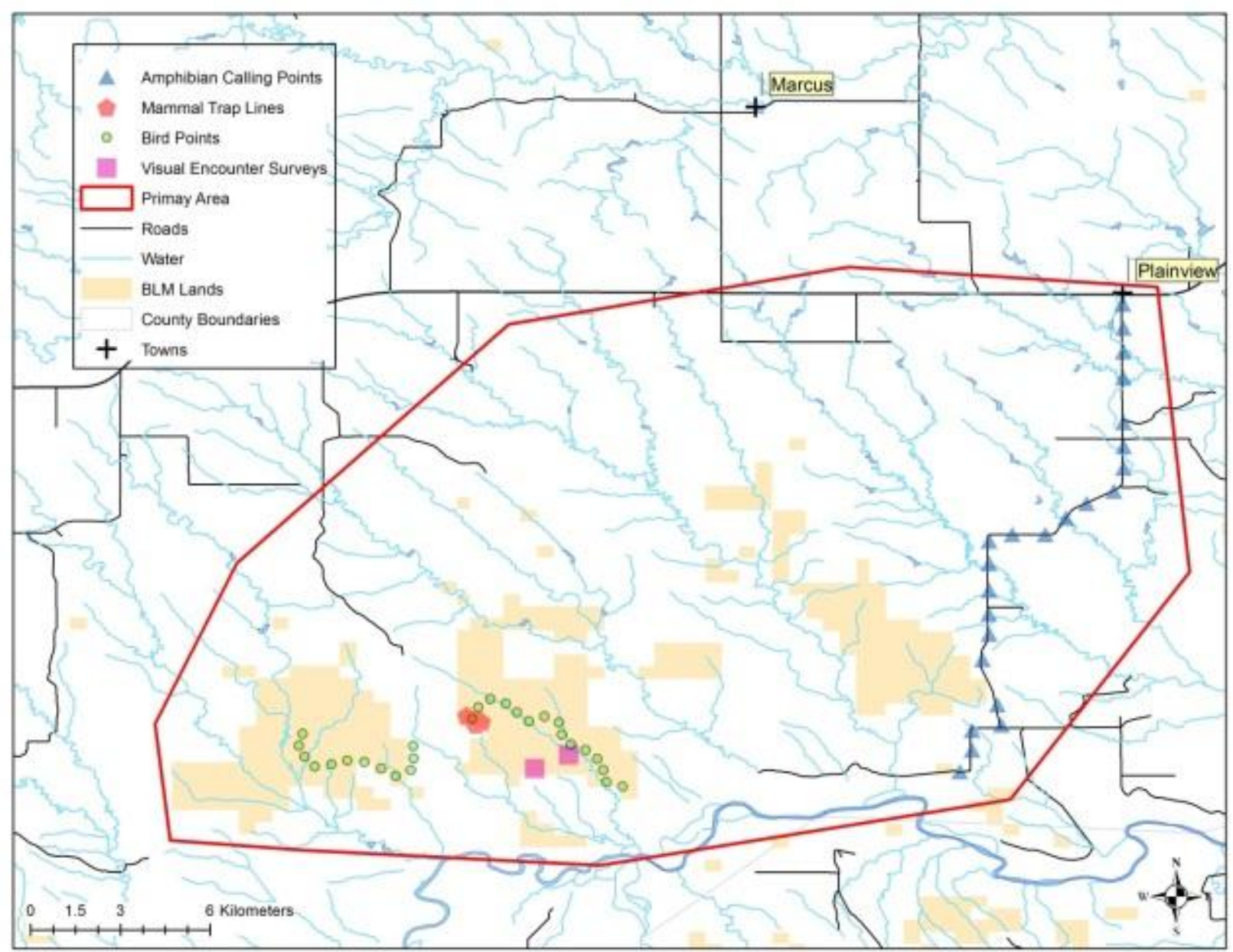

Figure 9. Two Rivers primary area survey locations 


\section{METHODS}

Between May and September of 2014, we conducted surveys to document distribution, habitat associations, and status of bird, terrestrial small mammal, bat, amphibian, and reptile species across the eight primary survey areas. Within each primary area, we surveyed for bird species by conducting on- and off-road point count transects. We surveyed for terrestrial small mammals by setting trap lines using a variety of traps to maximize diversity of species detected. To document bat species, we placed long-term acoustic monitoring devices at 5 sentinel areas. We documented amphibian species at listening stations along road transects during early summer rain events and amphibians and aquatic reptiles with visual encounter surveys in wetlands. All species encountered incidentally during the course of this study were recorded to further increase distribution information for species not documented in structured surveys.

\section{BIRD SURVEYS}

To document the status and distribution of bird species within the study area, we conducted point counts using on- and off-road transects in May and June of 2014. For the road-based transects, we selected road routes that maximized the amount of BLM lands the road passed through and surveyed 24 points spaced $0.8 \mathrm{~km}$ apart over $18.4 \mathrm{~km}$. In areas with little road access through BLM managed lands, we conducted off-road point counts by walking between points spaced $0.4 \mathrm{~km}$ apart with transects ranging from 3 to 16 points in length. At both on- and off-road transects, we surveyed from sunrise until we reached the desired number of points or until bird activity started to diminish around 10:00 a.m., whichever was occurred first. At each point, we surveyed for 10 minutes, recording the species and distance to each bird detected (sensu Hendricks et al. 2008). We also recorded nests or young detected as we moved between points.

\section{Small Mammal Surveys}

To document small mammal species present on BLM managed lands, we placed trap lines through a variety of habitats in each primary area (sensu Hendricks et al. 2007). Within each primary area we attempted to place trap lines in all distinct habitat types present. When the option to place multiple lines in one habitat type existed, we prioritized riparian areas and areas with tree or shrub cover since we expected these areas to support a higher diversity and abundance of small mammals. Along each trap line we designated 10 stations spaced $10 \mathrm{~m}$ apart, over $90 \mathrm{~m}$. At each station we deployed an array of traps. We placed a cylindrical pit fall container (depth $20 \mathrm{~cm}$, diameter $15 \mathrm{~cm}$ ) at the center of the station and surrounded it at about $1 \mathrm{~m}$ distance in 3 cardinal directions with a Sherman trap $(7.5 \times$ $8.8 \times 22.7-\mathrm{cm}$ folding, H.B. Sherman Traps, Inc., Tallahassee, FL), a Museum Special snap trap (Forestry Suppliers, Jackson, MS), and a Victor snap trap (Woodstream Corporation, Lititz, PA). We baited Victor and Museum Special traps with peanut butter and the Sherman traps with commercial sweet feed. To ensure the welfare of animals captured in Sherman traps on cold nights, we also placed a small amount of synthetic quilt batting within each trap to allow animals to build nests if needed. If an animal was injured by the trap or during handling, we euthanized it using isoflurane (University of 
Montana Institutional Animal Care and Use Committee protocol 025-13BMMNHP-050613).

Captured animals were identified in hand if possible based on morphological characteristics and pelage. If we were uncertain of the species of an individual specimen, we euthanized the individual and collected it as a voucher specimen for more detailed morphological analysis (Foresman 2012) at the Montana State University Zoological Museum in Bozeman, MT.

\section{BAT SURVEYS}

To document bat species present in the study area we placed SM2 BAT+ ultrasonic acoustic detectors (Wildlife Acoustics, Maynard, MA) near habitat features like reservoirs and rivers known to attract a diversity of bat species. Due to logistic constraints we only placed detectors in 3 of the 8 primary areas. SM2 BAT+ ultrasonic detectors can be left in the field over long periods of time on solar panels and deep cycle batteries so we deployed these detectors in late October of 2013 and plan to gather data through at least the fall of 2015; downloading data, checking system power, and ensuring that microphones are fully functional every two to four months. We used Sonobat (SonoBat Version 3.0 Montana, SonoBat, Arcata, CA) to identify acoustic recordings to species using Echolocation Call Characteristics of Montana Bats (Appendix B).

\section{AMPHIBIAN AND REPTILE SURVEYS}

We surveyed for amphibians using nighttime call station transects along roads after rains in the spring and early summer to detect breeding calls of adults (Heyer et al. 1994). We focused our call survey routes along roads that passed through and in close proximity to BLM lands, stopping to listen for calling amphibians at approximately $0.8 \mathrm{~km}$ intervals along each transect and recording the species detected, estimated number in each breeding chorus, and direction and distance to each breeding chorus.

We also conducted visual encounter surveys of wetlands on BLM managed lands using dipnets during the summer to detect adults and larvae (Heyer et al. 1994). These surveys supplemented the call surveys which may have missed Western Tiger Salamanders (Ambystoma mavortium) which do not vocalize and Northern Leopard Frogs (Lithobates pipiens) which breed in the early spring and may have been missed during our call survey efforts in some areas (Werner et al. 2004). To conduct these surveys, we walked the edge of the reservoir or wetland looking for amphibians and reptiles and used a dip net in shallow waters $(<0.5 \mathrm{~m})$ to capture and identify amphibian larvae.

\section{INCIDENTAL OBSERVATIONS}

We recorded species incidentally encountered throughout our time in western South Dakota in order to document distribution information for species that were undetectable with the structured survey methodologies we employed (e.g. large mammals).

\section{ANALYSIS}

For each primary survey area, we documented the species present, basic habitat associations, as well as baseline indices for relative spatial distribution and status. For birds, we tabulated the proportion of points we detected each species at within each of the major habitat types in each primary survey area as well as the average number of individuals detected at points the species was detected.

For small mammals, we tabulated the proportion of lines we detected each species at within each of the major habitat types in each 
primary survey area as well as the catch per unit effort for each trap type (i.e. the number of individuals captured by a particular trap type divided by the number of available traps over all nights of trapping).

For bats, we tabulated monthly presence of individual species throughout the time ultrasonic detectors were deployed at each of the long-term monitoring sites as well as the overall amount of bat activity each month, the number of nights with bat activity, and the average, standard error, and range of the number of passes per night each month across all bat species.

For amphibian calling surveys, we tabulated the proportion of points a species was detected on, as well as the average estimated number of individuals calling at points where they were detected. Because we conducted visual encounter surveys at wetlands late in the summer after some amphibian species had metamorphosed and dispersed from breeding sites, we summarized that data with incidental observation data.

\section{Availability of Data}

All structured survey locations and locations of detections of animals during surveys or made incidentally is available online through the Montana Natural Heritage Program's MapViewer http://mtnhp.org/mapviewer/ so that is integrated with other survey and incidental observation data and more readily available for resource management plans and project-level planning. 


\section{RESULTS}

\section{BIRDS}

Over all 8 primary areas we conducted 19 transect surveys, with 7 road transects and 12 walking transects (Table 1). In total we surveyed 278 points with 148 along road transects and 130 along walking transects. Road transects averaged $16 \mathrm{~km}$ in length, with 21 points. Walking transects averaged $4 \mathrm{~km}$ in length, with 11 points.

Table 1. Number of bird transects and points surveyed in each primary area on driving and walking transects.

\begin{tabular}{|l|c|c|c|}
\hline Primary Area & $\begin{array}{c}\text { No. } \\
\text { Driving } \\
\text { Transects } \\
\text { (Points) }\end{array}$ & $\begin{array}{c}\text { No. Walking } \\
\text { Transects } \\
\text { (Points) }\end{array}$ & $\begin{array}{c}\text { Total No. } \\
\text { Transects } \\
\text { (Points) }\end{array}$ \\
\hline Butte County & $2(48)$ & $2(13)$ & $4(61)$ \\
\hline Newell & $1(24)$ & $2(29)$ & $3(53)$ \\
\hline Fort Meade & $1(24)$ & 0 & $1(24)$ \\
\hline Lead & 0 & $1(10)$ & $1(10)$ \\
\hline S. Black Hills & $1(12)$ & $3(21)$ & $4(33)$ \\
\hline Pedro & $1(16)$ & $2(30)$ & $3(46)$ \\
\hline $\begin{array}{l}\text { Mission } \\
\text { Ridge }\end{array}$ & $1(24)$ & 0 & $1(24)$ \\
\hline Two Rivers & 0 & $2(27)$ & $2(27)$ \\
\hline \multicolumn{1}{|c|}{ Totals } & $\mathbf{7 ( 1 4 8 )}$ & $\mathbf{1 2 ( 1 3 0 )}$ & $\mathbf{1 9 ( 2 7 8 )}$ \\
\hline
\end{tabular}

During Point count surveys we detected 100 species of birds, 11 of which are listed as Sensitive by the BLM (Table 2) and 1 of which is on South Dakota's Rare, Threatened or Endangered Animals list (SDNHP 2014). We detected the highest diversity of bird species in Central South Dakota, where the prairie transitions to conifer forests, and the lowest diversity within the conifer forests of the Black Hills.

In the Butte County primary area, we conducted point counts in mixedgrass prairie with occasional sparse sagebrush cover and stock reservoirs, and within riparian woodlands along ephemeral creeks. During the road transect surveys we detected 30 species, 2 of which are
BLM Sensitive (Table C-1). While conducting walking transect surveys, we detected 30 species, 2 of which are BLM Sensitive (Table C2).

In the Newell Primary area, we conducted point counts in mixedgrass prairie. During road transect surveys we detected 28 species, 4 of which are BLM Sensitive (Table C-3). During the walking transect surveys we detected 25 species, 2 of which are BLM Sensitive (Table C4).

In the Fort Meade primary area, we conducted point counts in mixedgrass prairie, coniferous forest, and deciduous woodland. We detected 47 species, 1 of which are BLM Sensitive (Table C-5).

In the Lead primary area, we conducted point counts in coniferous forest, detecting 16 species (Table C-6). This species count is likely low as there was occasional heavy rain over the duration of the survey, and we concluded the survey early due to decreased bird activity.

In the Southern Black Hills primary area, we conducted surveys in mixedgrass prairie, sparse coniferous woodland dominated by ponderosa pine and juniper, and deciduous riparian woodland. We detected 25 species during the road survey (Table C-7). During the walking transect surveys we detected 32 species (Table C-8).

In the Pedro primary area, we surveyed points in mixedgrass prairie and prairie areas adjacent to deciduous woodlands, and juniper woodlands. While conducting the road based 
survey, we detected 36 species, 3 of which are BLM Sensitive with one also listed as a South Dakota Rare, Threatened, or Endangered Animal (Table C-9, SDNHP 2014). During the walking surveys we detected 37 species, including 2 that are BLM Sensitive (Table C-10).

In the Mission Ridge primary area we surveyed points in mixedgrass prairie, detecting 28 species, 2 of which are listed as BLM Sensitive (Table C-11).

In the Two Rivers primary area we surveyed points in juniper woodlands and grasslands within badlands. During these surveys we detected 31 species, 1 of which is BLM Sensitive (Table C-12).

Throughout the field season we recorded 1,015 incidental observations of 111 unique bird species, including 25 not detected during point counts; 5 of which are BLM Sensitive (Table 2).

Table 2. Bird species detected during structured surveys and incidentally. Numbers represent the number of individuals detected. Global ranks are listed, along with the state ranks of rare, threatened, and endangered species. Species denoted with an * are BLM Sensitive species.

\begin{tabular}{|c|c|c|c|c|}
\hline $\begin{array}{c}\text { Bird } \\
\text { Species }\end{array}$ & 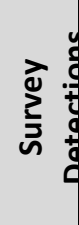 & 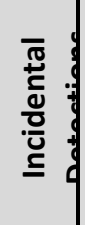 & 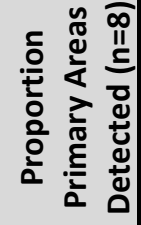 & 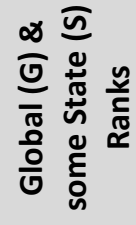 \\
\hline \multicolumn{5}{|l|}{ Cormorants } \\
\hline $\begin{array}{l}\text { Double-crested } \\
\text { Cormorant }\end{array}$ & 0 & 1 & 0.12 & G5 \\
\hline \multicolumn{5}{|l|}{ Waterfowl } \\
\hline $\begin{array}{l}\text { American } \\
\text { Wigeon }\end{array}$ & 2 & 11 & 0.25 & G5 \\
\hline $\begin{array}{l}\text { Blue-winged } \\
\text { Teal }\end{array}$ & 3 & 27 & 0.62 & G5 \\
\hline Canvasback & 0 & 2 & 0.12 & G5 \\
\hline Gadwall & 1 & 9 & 0.5 & G5 \\
\hline $\begin{array}{l}\text { Green-winged } \\
\text { Teal }\end{array}$ & 0 & 4 & 0.25 & G5 \\
\hline
\end{tabular}

\begin{tabular}{|c|c|c|c|c|}
\hline Mallard & 18 & 31 & 0.62 & G5 \\
\hline Northern Pintail & 1 & 5 & 0.38 & G5 \\
\hline $\begin{array}{l}\text { Northern } \\
\text { Shoveler }\end{array}$ & 0 & 12 & 0.25 & G5 \\
\hline Ruddy Duck & 0 & 1 & 0.12 & G5 \\
\hline Wood Duck & 0 & 1 & 0.12 & G5 \\
\hline Canada Goose & 16 & 6 & 0.5 & G5 \\
\hline \multicolumn{5}{|l|}{ Falcons } \\
\hline $\begin{array}{l}\text { American } \\
\text { Kestrel }\end{array}$ & 7 & 14 & 0.75 & G5 \\
\hline Prairie Falcon & 0 & 4 & 0.38 & G5 \\
\hline Merlin & 2 & 2 & 0.38 & G5 \\
\hline \multicolumn{5}{|l|}{ Raptors } \\
\hline Red-tailed Hawk & 3 & 6 & 0.75 & G5 \\
\hline Golden Eagle * & 6 & 8 & 0.5 & $\begin{array}{c}\text { S3S4B,S3 } \\
\text { N /G5 }\end{array}$ \\
\hline $\begin{array}{l}\text { Northern } \\
\text { Harrier }\end{array}$ & 2 & 2 & 0.38 & G5 \\
\hline $\begin{array}{l}\text { Ferruginous } \\
\text { Hawk* }\end{array}$ & 1 & 4 & 0.25 & $\begin{array}{c}\text { S4B,SZN } \\
\text { /G4 }\end{array}$ \\
\hline $\begin{array}{l}\text { Swainson's } \\
\text { Hawk }\end{array}$ & 2 & 0 & 0.12 & $\begin{array}{c}\text { S4B,SZN } \\
\text { /G5 }\end{array}$ \\
\hline Bald Eagle * & 1 & 5 & 0.25 & $\begin{array}{c}\text { S1B,S2N } \\
/ \text { G5 }\end{array}$ \\
\hline \multicolumn{5}{|l|}{ Nighthawks } \\
\hline $\begin{array}{l}\text { Common } \\
\text { Nighthawk }\end{array}$ & 11 & 20 & 0.88 & G5 \\
\hline \multicolumn{5}{|l|}{ Owls } \\
\hline Burrowing Owl* & 2 & 2 & 0.38 & $\begin{array}{c}\text { S3S4B,SZN } \\
\text { /G4 }\end{array}$ \\
\hline Short-eared Owl & 1 & 0 & 0.12 & G5 \\
\hline $\begin{array}{l}\text { Great Horned } \\
\text { Owl }\end{array}$ & 0 & 7 & 0.25 & G5 \\
\hline \multicolumn{5}{|l|}{ Pelicans } \\
\hline $\begin{array}{l}\text { American White } \\
\text { Pelican }\end{array}$ & 3 & 1 & 0.38 & $\begin{array}{c}\text { S3B,SZN } \\
\text { /G4 }\end{array}$ \\
\hline \multicolumn{5}{|l|}{ Herons } \\
\hline $\begin{array}{l}\text { Great Blue } \\
\text { Heron }\end{array}$ & 3 & 6 & 0.62 & $\begin{array}{c}\text { S4B,SZN } \\
\text { /G5 }\end{array}$ \\
\hline \multicolumn{5}{|l|}{$\begin{array}{l}\text { Pigeons/Dove } \\
s\end{array}$} \\
\hline Rock Pigeon & 1 & 0 & 0.12 & G5 \\
\hline Mourning Dove & 156 & 42 & 0.88 & G5 \\
\hline \multicolumn{5}{|l|}{ Rails } \\
\hline American Coot & 1 & 8 & 0.38 & G5 \\
\hline Shorebirds & & & & \\
\hline
\end{tabular}




\begin{tabular}{|c|c|c|c|c|}
\hline Killdeer & 33 & 40 & 0.75 & G5 \\
\hline $\begin{array}{l}\text { Upland } \\
\text { Sandpiper }\end{array}$ & 80 & 43 & 0.88 & G5 \\
\hline $\begin{array}{l}\text { Long-billed } \\
\text { Curlew * }\end{array}$ & 4 & 4 & 0.38 & $\begin{array}{c}\text { S3B,SZN } \\
\text { /G5 }\end{array}$ \\
\hline Marbled Godwit & 0 & 2 & 0.25 & G5 \\
\hline $\begin{array}{l}\text { Spotted } \\
\text { Sandpiper }\end{array}$ & 1 & 6 & 0.38 & G5 \\
\hline $\begin{array}{l}\text { Wilson's } \\
\text { Phalarope }\end{array}$ & 8 & 16 & 0.38 & G5 \\
\hline Wilson's Snipe & 2 & 0 & 0.12 & G5 \\
\hline $\begin{array}{l}\text { American } \\
\text { Avocet }\end{array}$ & 0 & 2 & 0.25 & G5 \\
\hline \multicolumn{5}{|l|}{ Gulls } \\
\hline Ring-billed Gull & 5 & 2 & 0.38 & G5 \\
\hline \multicolumn{5}{|l|}{ Blackbirds } \\
\hline Bobolink & 17 & 4 & 0.75 & G5 \\
\hline $\begin{array}{l}\text { Brown-headed } \\
\text { Cowbird }\end{array}$ & 168 & 25 & 0.88 & G5 \\
\hline $\begin{array}{l}\text { Brewer's } \\
\text { Blackbird }\end{array}$ & 9 & 5 & 0.25 & G5 \\
\hline $\begin{array}{l}\text { Eastern } \\
\text { Meadowlark }\end{array}$ & 0 & 1 & 0.12 & $\begin{array}{c}\text { S2B,SZN } \\
/ G 5\end{array}$ \\
\hline $\begin{array}{l}\text { Western } \\
\text { Meadowlark }\end{array}$ & 231 & 80 & 0.88 & G5 \\
\hline $\begin{array}{l}\text { Common } \\
\text { Grackle }\end{array}$ & 19 & 10 & 0.62 & G5 \\
\hline Orchard Oriole & 4 & 10 & 0.5 & G5 \\
\hline $\begin{array}{l}\text { Red-winged } \\
\text { Blackbird }\end{array}$ & 91 & 40 & 1 & G5 \\
\hline $\begin{array}{l}\text { Yellow-headed } \\
\text { Blackbird }\end{array}$ & 4 & 3 & 0.38 & G5 \\
\hline \multicolumn{5}{|l|}{ Chicadees } \\
\hline $\begin{array}{l}\text { Black-capped } \\
\text { Chickadee }\end{array}$ & 27 & 10 & 0.62 & G5 \\
\hline \multicolumn{5}{|l|}{ Finches } \\
\hline Pine Siskin & 0 & 4 & 0.25 & G5 \\
\hline $\begin{array}{l}\text { American } \\
\text { Goldfinch }\end{array}$ & 11 & 10 & 0.62 & G5 \\
\hline Red Crossbill & 0 & 1 & 0.12 & G5 \\
\hline \multicolumn{5}{|l|}{ Flycatchers } \\
\hline Eastern Kingbird & 26 & 26 & 0.88 & G5 \\
\hline $\begin{array}{l}\text { Scissor-tailed } \\
\text { Flycatcher }\end{array}$ & 0 & 1 & 0.12 & G5 \\
\hline $\begin{array}{l}\text { Western } \\
\text { Kingbird }\end{array}$ & 16 & 11 & 0.62 & G5 \\
\hline $\begin{array}{l}\text { Dusky } \\
\text { Flycatcher }\end{array}$ & 1 & 0 & 0.12 & G5 \\
\hline $\begin{array}{l}\text { Great Crested } \\
\text { Flycatcher }\end{array}$ & 0 & 1 & 0.12 & G5 \\
\hline
\end{tabular}

\begin{tabular}{|c|c|c|c|c|}
\hline $\begin{array}{l}\text { Western Wood- } \\
\text { Pewee }\end{array}$ & 1 & 5 & 0.38 & G5 \\
\hline $\begin{array}{l}\text { Cordilleran } \\
\text { Flycatcher }\end{array}$ & 0 & 1 & 0.12 & G5 \\
\hline Alder Flycatcher & 0 & 1 & 0.12 & G5 \\
\hline $\begin{array}{l}\text { Willow } \\
\text { Flycatcher }\end{array}$ & 2 & 2 & 0.25 & G5 \\
\hline \multicolumn{5}{|l|}{ Gnatcatchers } \\
\hline $\begin{array}{l}\text { Blue-gray } \\
\text { Gnatcatcher * }\end{array}$ & 1 & 0 & 0.12 & $\begin{array}{c}\text { S1B,SZN } \\
/ G 5\end{array}$ \\
\hline \multicolumn{5}{|l|}{$\begin{array}{l}\text { Jays/Crows/ } \\
\text { Magpies }\end{array}$} \\
\hline American Crow & 43 & 8 & 0.62 & G5 \\
\hline Blue Jay & 6 & 8 & 0.5 & G5 \\
\hline $\begin{array}{l}\text { Black-billed } \\
\text { Magpie }\end{array}$ & 23 & 6 & 0.38 & G5 \\
\hline \multicolumn{5}{|l|}{ Kinglets } \\
\hline $\begin{array}{l}\text { Golden- } \\
\text { crowned Kinglet }\end{array}$ & 0 & 1 & 0.12 & G5 \\
\hline $\begin{array}{l}\text { Ruby-crowned } \\
\text { Kinglet } \\
\end{array}$ & 9 & 1 & 0.12 & G5 \\
\hline \multicolumn{5}{|l|}{ Larks } \\
\hline Horned Lark & 69 & 35 & 0.88 & G5 \\
\hline \multicolumn{5}{|l|}{ Longspurs } \\
\hline $\begin{array}{l}\text { Chestnut- } \\
\text { collared } \\
\text { Longspur* }\end{array}$ & 21 & 12 & 0.25 & G5 \\
\hline \multicolumn{5}{|l|}{ Nuthatches } \\
\hline $\begin{array}{l}\text { White-breasted } \\
\text { Nuthatch }\end{array}$ & 1 & 2 & 0.12 & G5 \\
\hline $\begin{array}{l}\text { Red-breasted } \\
\text { Nuthatch } \\
\end{array}$ & 12 & 13 & 0.5 & G5 \\
\hline \multicolumn{5}{|l|}{ Pipits } \\
\hline Sprague's Pipit* & 1 & 1 & 0.12 & $\begin{array}{c}\text { S2B,SZN } \\
/ G 4\end{array}$ \\
\hline \multicolumn{5}{|l|}{ Shrikes } \\
\hline $\begin{array}{l}\text { Loggerhead } \\
\text { Shrike* }\end{array}$ & 5 & 9 & 0.38 & G4 \\
\hline \multicolumn{5}{|l|}{$\begin{array}{l}\text { Sparrows/ } \\
\text { Buntings }\end{array}$} \\
\hline $\begin{array}{l}\text { Baird's } \\
\text { Sparrow* }\end{array}$ & 0 & 1 & 0.12 & $\begin{array}{c}\text { S2B,SZN } \\
/ \mathrm{G} 4\end{array}$ \\
\hline Lark Sparrow & 80 & 21 & 0.88 & G5 \\
\hline $\begin{array}{l}\text { Grasshopper } \\
\text { Sparrow }\end{array}$ & 91 & 27 & 0.88 & G5 \\
\hline Vesper Sparrow & 0 & 16 & 0.5 & G5 \\
\hline $\begin{array}{l}\text { Brewer's } \\
\text { Sparrow * }\end{array}$ & 0 & 1 & 0.12 & G5 \\
\hline
\end{tabular}




\begin{tabular}{|c|c|c|c|c|}
\hline Lark Bunting & 124 & 54 & 0.75 & G5 \\
\hline Field Sparrow & 54 & 12 & 0.62 & G5 \\
\hline $\begin{array}{l}\text { Chipping } \\
\text { Sparrow }\end{array}$ & 25 & 11 & 0.62 & G5 \\
\hline Dark-eyed Junco & 3 & 2 & 0.25 & G5 \\
\hline Spotted Towhee & 63 & 10 & 0.75 & G5 \\
\hline Song Sparrow & 7 & 2 & 0.25 & G5 \\
\hline \multicolumn{5}{|l|}{ Starlings } \\
\hline $\begin{array}{l}\text { European } \\
\text { Starling }\end{array}$ & 21 & 3 & 0.62 & G5 \\
\hline \multicolumn{5}{|l|}{ Swallows } \\
\hline Tree Swallow & 11 & 3 & 0.62 & G5 \\
\hline Cliff Swallow & 5 & 0 & 0.12 & G5 \\
\hline $\begin{array}{l}\text { Northern } \\
\text { Rough-winged } \\
\text { Swallow }\end{array}$ & 21 & 7 & 0.62 & G5 \\
\hline Barn Swallow & 32 & 15 & 0.62 & G5 \\
\hline \multicolumn{5}{|l|}{$\begin{array}{l}\text { Tanagers/ } \\
\text { Cardinals/ } \\
\text { Buntings }\end{array}$} \\
\hline $\begin{array}{l}\text { Black-headed } \\
\text { Grosbeak }\end{array}$ & 5 & 0 & 0.25 & G5 \\
\hline Blue Grosbeak & 13 & 11 & 0.38 & G5 \\
\hline $\begin{array}{l}\text { Western } \\
\text { Tanager }\end{array}$ & 4 & 2 & 0.38 & G5 \\
\hline Lazuli Bunting & 2 & 0 & 0.12 & G5 \\
\hline Dickcissel & 20 & 6 & 0.38 & G5 \\
\hline \multicolumn{5}{|l|}{$\begin{array}{l}\text { Thrashers } \\
\text { Mockingbirds } \\
\text { / Catbirds }\end{array}$} \\
\hline Gray Catbird & 1 & 0 & 0.12 & G5 \\
\hline Sage Thrasher * & 0 & 1 & 0.12 & $\begin{array}{c}\text { S2B,SZN } \\
\text { /G5 }\end{array}$ \\
\hline Brown Thrasher & 6 & 16 & 0.75 & G5 \\
\hline \multicolumn{5}{|l|}{ Thrushes } \\
\hline $\begin{array}{l}\text { Mountain } \\
\text { Bluebird }\end{array}$ & 5 & 3 & 0.25 & G5 \\
\hline Eastern Bluebird & 1 & 1 & 0.12 & G5 \\
\hline $\begin{array}{l}\text { Swainson's } \\
\text { Thrush }\end{array}$ & 2 & 1 & 0.25 & G5 \\
\hline $\begin{array}{l}\text { Townsend's } \\
\text { Solitaire }\end{array}$ & 0 & 1 & 0.12 & G5 \\
\hline American Robin & 31 & 12 & 0.62 & G5 \\
\hline \multicolumn{5}{|l|}{ Vireos } \\
\hline Bell's Vireo & 2 & 2 & 0.38 & G5 \\
\hline Red-eyed Vireo & 2 & 1 & 0.25 & G5 \\
\hline
\end{tabular}

\begin{tabular}{|c|c|c|c|c|}
\hline $\begin{array}{l}\text { Plumbeous } \\
\text { Vireo }\end{array}$ & 1 & 0 & 0.12 & G5 \\
\hline \multicolumn{5}{|l|}{ Warblers } \\
\hline Yellow Warbler & 18 & 9 & 0.88 & G5 \\
\hline $\begin{array}{l}\text { Yellow-breasted } \\
\text { Chat }\end{array}$ & 4 & 1 & 0.38 & G5 \\
\hline $\begin{array}{l}\text { Common } \\
\text { Yellowthroat }\end{array}$ & 4 & 2 & 0.38 & G5 \\
\hline $\begin{array}{l}\text { Yellow-rumped } \\
\text { Warbler }\end{array}$ & 5 & 3 & 0.12 & G5 \\
\hline Ovenbird & 4 & 0 & 0.25 & G5 \\
\hline \multicolumn{5}{|l|}{ Waxwings } \\
\hline Cedar Waxwing & 8 & 5 & 0.62 & G5 \\
\hline \multicolumn{5}{|l|}{ Wrens } \\
\hline House Wren & 14 & 2 & 0.38 & G5 \\
\hline Canyon Wren & 1 & 1 & 0.12 & G5 \\
\hline Rock Wren & 12 & 5 & 0.5 & G5 \\
\hline \multicolumn{5}{|l|}{$\begin{array}{l}\text { Upland Game } \\
\text { Birds }\end{array}$} \\
\hline $\begin{array}{l}\text { Ring-necked } \\
\text { Pheasant }\end{array}$ & 23 & 0 & 0.38 & G5 \\
\hline $\begin{array}{l}\text { Greater Sage- } \\
\text { Grouse* }\end{array}$ & 0 & 1 & 0.12 & G3G4 \\
\hline $\begin{array}{l}\text { Sharp-tailed } \\
\text { Grouse }\end{array}$ & 0 & 3 & 0.25 & G5 \\
\hline Wild Turkey & 8 & 3 & 0.38 & G5 \\
\hline \multicolumn{5}{|l|}{ Woodpeckers } \\
\hline $\begin{array}{c}\text { Red-headed } \\
\text { Woodpecker* }\end{array}$ & 1 & 3 & 0.38 & G5 \\
\hline Northern Flicker & 19 & 15 & 0.88 & G5 \\
\hline $\begin{array}{c}\text { Hairy } \\
\text { Woodpecker }\end{array}$ & 1 & 2 & 0.25 & G5 \\
\hline $\begin{array}{c}\text { Red-naped } \\
\text { Sapsucker }\end{array}$ & 2 & 2 & 0.12 & G5 \\
\hline \multicolumn{5}{|l|}{ Vultures } \\
\hline Turkey Vulture & 5 & 12 & 0.62 & G5 \\
\hline
\end{tabular}




\section{MAMMALS}

Across the survey area, we placed 47 trap-lines (Table 3). We trapped most lines for three nights; one line in the Newell primary area had to be removed after one night due to cattle damage.

Table 3. Number of small mammal traplines and bat acoustic detectors deployed over all primary areas.

\begin{tabular}{|l|c|c|}
\hline \multicolumn{1}{|c|}{ Primary Area } & $\begin{array}{c}\text { Number of } \\
\text { Traplines }\end{array}$ & $\begin{array}{c}\text { Number of } \\
\text { Bat Detectors }\end{array}$ \\
\hline Butte County & 11 & 2 \\
\hline Newell & 6 & 2 \\
\hline Fort Meade & 5 & 1 \\
\hline Lead & 5 & 0 \\
\hline S. Black Hills & 5 & 0 \\
\hline Pedro & 5 & 0 \\
\hline Mission Ridge & 5 & 0 \\
\hline Two Rivers & 5 & 0 \\
\hline \multicolumn{1}{|c|}{ Totals } & $\mathbf{4 7}$ & $\mathbf{5}$ \\
\hline
\end{tabular}

We deployed small mammal traplines between mid-August and mid-September 2014. Across all primary areas we captured 15 species of small mammals, none of which are listed as sensitive by the BLM, or as a rare, threatened, or endangered animal (Table 4, SDNHP 2014). We were able to identify all species in hand except for one shrew (Sorex spp.) caught in a sagebrush shrubland. After examination of cranial morphology in a zoological museum we feel reasonably confident that this is a Montane Shrew (S. monticolus) or Dwarf Shrew (S. nanus), but are awaiting the results of a detailed examination of its guard hairs (sensu Pocock and Jennings 2006) before making a final decision on the species identification.

We detected the greatest diversity of small mammal species in the conifer forests of the Black Hills, while mammal communities were the least diverse in the mixedgrass prairies of west central South Dakota.

In the Butte County primary area, we placed traplines in mixedgrass prairie, barren habitat, shrublands, and along an ephemeral creek, detecting 6 species (Tables D-1, D-2).

In the Newell primary area, we set traplines in mixedgrass prairie detecting 3 species (Tables D-3, D-4).

In the Fort Meade primary area, we placed traplines in mixedgrass prairie, herbaceous wetland, deciduous woodland dominated by cottonwood, green ash and bur oak, and ponderosa pine woodland, detecting 4 species (Tables D-5, D-6).

In the Lead primary area, we placed traplines in coniferous woodland, a wet meadow, and a woody riparian area adjacent to a creek, detecting 7 species (Tables D-7, D-8).

In the southern Black Hills primary area at the Fossil Cycad National Monument, we placed traplines in mixed grass prairie and ponderosa pine forest, detecting 4 species (Tables D-9, D10).

In the Pedro primary area, we placed traplines in shrublands dominated by silver sagebrush and yucca, coniferous woodland dominated by rocky mountain juniper, and a coulee bottom dominated by mixed grass prairie, detecting 5 species (Tables D-11, D-12).

In the Mission Ridge primary area, we placed traplines in mixedgrass prairie, barren badlands, and a shrubland, detecting 3 species (Tables D13, D-14).

In the Two Rivers primary area, we placed traplines in barren badlands, coniferous 
woodland dominated by ponderosa pine, and mixedgrass prairie capturing 5 species (Tables D-15, D-16).

We deployed 5 SM2 acoustic bat detectors in 3 primary areas in October 2013 and intend to have them deployed through the fall of 2015. We analyzed and summarized echolocation call information through August 2014 for this report. In the Butte County primary area, we placed one detector next to a stock reservoir next to Old Highway 85 near Battle Creek and placed the other on a bench next to the South Fork of the Moreau River near the Harding Road. In the Newell primary area, we placed one detector on a small ridge overlooking Jug Creek and the other detector next to the Belle Fourche River by the Bismarck Bridge. In the Fort Meade primary area we placed a detector next to Fort Meade Reservoir.

Across all primary survey areas, we definitively identified echolocation calls of 6 species with bat activity in October and November 2013 and between April and August 2014; no bat activity was detected in December of 2013 or January, February, or March of 2014 (Tables E-1, E-2).

In the Butte County primary area, we detected 5 bat species, 4 at the Battle Creek detector and 3 at the South Fork of the Moreau River detector (Table E-1). At Battle Creek, we detected bat activity between May and August 2014 with the greatest amount of activity recorded in May and then decreasing through August (Table E-2). At the South Fork of the Moreau River, we detected bats between April and August 2014 with the greatest amount of activity in August (Table E-2).

In the Newell primary area, we detected a total of 6 bat species, 5 at the Jug Creek detector and 6 at the Bismarck Bridge detector (Table E-1). At
Jug Creek, we detected bats between May and August 2014 (Table E-2). At Bismarck Bridge, we detected bats between April and August 2014 (Table E-2). At both stations bat activity was greatest in July 2014 (Table E-2).

In the Fort Meade primary area, we detected 5 bat species at the Fort Meade Reservoir with bat activity in October and November 2013 and between April and August 2014 (Tables E-1, E2). Bat activity was greatest and relatively constant in May, June, and July of 2014 (Table E-2).

Throughout the field season we recorded 120 incidental observations of 20 unique mammal species, including 17 not detected at traplines or bat acoustic detectors; one of these is BLM Sensitive (Table 4).

Table 4. Mammal species detected within the survey area during structured surveys and incidentally. Columns display the number of traplines where each species was detected, the number of point observations of each species, and the proportion of primary areas within which each species was detected. Bat species that could be definitively identified by echolocation calls are denoted with a “D”. Global ranks are listed, along with the state ranks of rare, threatened and endangered species. Species denoted with a * are BLM Sensitive species.

\begin{tabular}{|c|c|c|c|c|}
\hline $\begin{array}{l}\text { Mammal } \\
\text { Species }\end{array}$ & 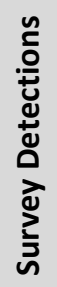 & 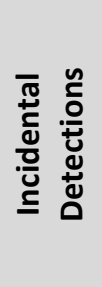 & 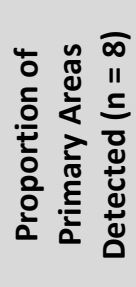 & 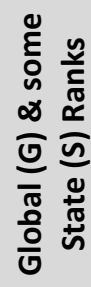 \\
\hline \multicolumn{5}{|l|}{ Bats } \\
\hline Big Brown Bat & D & 0 & $\begin{array}{l}0.66 \\
(n=3)\end{array}$ & G5 \\
\hline $\begin{array}{c}\text { Silver-haired } \\
\text { Bat }\end{array}$ & D & 0 & $\begin{array}{c}1 \\
(n=3)\end{array}$ & $\begin{array}{c}\mathrm{S} 4 / \mathrm{G} \\
5\end{array}$ \\
\hline $\begin{array}{c}\text { Eastern Red } \\
\text { Bat }\end{array}$ & $\mathrm{D}$ & 0 & $\begin{array}{c}1 \\
(n=3)\end{array}$ & G5 \\
\hline Hoary Bat & D & 0 & $\begin{array}{c}0.66 \\
(n=3)\end{array}$ & G5 \\
\hline
\end{tabular}




\begin{tabular}{|c|c|c|c|c|}
\hline $\begin{array}{c}\text { Western } \\
\text { Small-footed } \\
\text { Myotis } \\
\end{array}$ & D & 0 & $\begin{array}{c}1 \\
(n=3)\end{array}$ & G5 \\
\hline $\begin{array}{c}\text { Little Brown } \\
\text { Myotis }\end{array}$ & D & 0 & $\begin{array}{c}1 \\
(n=3)\end{array}$ & G3 \\
\hline \multicolumn{5}{|l|}{ Shrews } \\
\hline Least Shrew & 1 & 0 & 0.12 & $\begin{array}{c}\text { S3 } \\
\text { /G5 }\end{array}$ \\
\hline $\begin{array}{l}\text { Masked } \\
\text { Shrew }\end{array}$ & 3 & 0 & 0.12 & G5 \\
\hline Sorex Spp. & 1 & 0 & 0.12 & \\
\hline \multicolumn{5}{|l|}{ Pocket Mice } \\
\hline $\begin{array}{c}\text { Hispid Pocket } \\
\text { Mouse }\end{array}$ & 5 & 0 & 0.25 & G5 \\
\hline \multicolumn{5}{|l|}{$\begin{array}{c}\text { Pocket } \\
\text { Gophers } \\
\end{array}$} \\
\hline $\begin{array}{l}\text { Northern } \\
\text { Pocket } \\
\text { Gopher }\end{array}$ & 0 & 18 & 0.75 & G5 \\
\hline \multicolumn{5}{|l|}{ Beavers } \\
\hline Beaver & 0 & 1 & 0.12 & G5 \\
\hline \multicolumn{5}{|l|}{ Porcupines } \\
\hline Porcupine & 0 & 1 & 0.12 & G5 \\
\hline \multicolumn{5}{|l|}{ Squirrels } \\
\hline $\begin{array}{l}\text { Black-tailed } \\
\text { Prairie Dog * }\end{array}$ & 0 & 25 & 0.62 & G4 \\
\hline $\begin{array}{c}\text { Thirteen- } \\
\text { lined Ground } \\
\text { Squirrel }\end{array}$ & 2 & 1 & 0.12 & G5 \\
\hline $\begin{array}{c}\text { Eastern Fox } \\
\text { Squirrel }\end{array}$ & 0 & 2 & 0.12 & G5 \\
\hline $\begin{array}{c}\text { Least } \\
\text { Chipmunk }\end{array}$ & 1 & 5 & 0.25 & G5 \\
\hline Red Squirrel & 0 & 11 & 0.38 & G5 \\
\hline \multicolumn{5}{|l|}{ Mice/Voles } \\
\hline $\begin{array}{c}\text { Long-tailed } \\
\text { Vole }\end{array}$ & 3 & 0 & 0.12 & G5 \\
\hline Prairie Vole & 8 & 0 & 0.5 & G5 \\
\hline Meadow Vole & 7 & 1 & 0.38 & G5 \\
\hline $\begin{array}{c}\text { Southern } \\
\text { Red-backed } \\
\text { Vole }\end{array}$ & 3 & 0 & 0.12 & G5 \\
\hline $\begin{array}{c}\text { Northern } \\
\text { Grasshopper } \\
\text { Mouse }\end{array}$ & 3 & 0 & 0.12 & G5 \\
\hline $\begin{array}{l}\text { White-footed } \\
\text { Mouse }\end{array}$ & 13 & 0 & 0.62 & G5 \\
\hline
\end{tabular}

\begin{tabular}{|c|c|c|c|c|}
\hline Deer Mouse & 35 & 0 & 1 & G5 \\
\hline $\begin{array}{c}\text { Western } \\
\text { Harvest } \\
\text { Mouse }\end{array}$ & 2 & 0 & 0.25 & G5 \\
\hline $\begin{array}{c}\text { Plains Harvest } \\
\text { Mouse }\end{array}$ & 4 & 0 & 0.5 & G5 \\
\hline \multicolumn{5}{|l|}{ Rabbits } \\
\hline $\begin{array}{l}\text { White-tailed } \\
\text { Jack Rabbit }\end{array}$ & 0 & 6 & 0.38 & G5 \\
\hline $\begin{array}{c}\text { Desert } \\
\text { Cottontail }\end{array}$ & 0 & 2 & 0.25 & G5 \\
\hline \multicolumn{5}{|l|}{ Skunks } \\
\hline Striped Skunk & 0 & 1 & 0.12 & G5 \\
\hline \multicolumn{5}{|l|}{ Weasels } \\
\hline Badger & 0 & 4 & 0.38 & G5 \\
\hline \multicolumn{5}{|l|}{ Raccoons } \\
\hline Raccoon & 0 & 6 & 0.12 & G5 \\
\hline \multicolumn{5}{|l|}{$\begin{array}{c}\text { Wolves/ } \\
\text { Coyotes/ } \\
\text { Foxes }\end{array}$} \\
\hline Coyote & 0 & 1 & 0.12 & G5 \\
\hline Red Fox & 0 & 1 & 0.12 & G5 \\
\hline \multicolumn{5}{|l|}{ Pronghorn } \\
\hline Pronghorn & 0 & 22 & 0.5 & G5 \\
\hline \multicolumn{5}{|l|}{$\begin{array}{c}\text { Deer/ } \\
\text { Moose/Elk }\end{array}$} \\
\hline Elk & 0 & 1 & 0.12 & G5 \\
\hline Mule Deer & 0 & 1 & 0.12 & G5 \\
\hline $\begin{array}{l}\text { White-tailed } \\
\text { Deer }\end{array}$ & 0 & 10 & 0.62 & G5 \\
\hline
\end{tabular}

\section{AMPHIBIANS AND REPTILES}

We surveyed 123 nighttime amphibian call survey stations on 6 call survey routes in 4 primary areas on rainy nights in late-May through mid-June 2014 (Table 5). Not all primary areas were surveyed due to a lack of wetlands in proximity to roads and unfavorably dry conditions. 
Table 5. Number of nighttime amphibian call surveys performed in each primary area.

\begin{tabular}{|l|c|}
\hline Primary Area & $\begin{array}{c}\text { Number of Call Survey Routes } \\
\text { (Points Surveyed) }\end{array}$ \\
\hline Butte County & $2(39)$ \\
\hline Newell & $1(12)$ \\
\hline Fort Meade & $1(18)$ \\
\hline Lead & 0 \\
\hline S. Black Hills & 0 \\
\hline Pedro & 0 \\
\hline Mission Ridge & $1(29)$ \\
\hline Two Rivers & $1(25)$ \\
\hline \multicolumn{1}{|c|}{ Totals } & $6(123)$ \\
\hline
\end{tabular}

Across all primary areas we detected 5 amphibian species, including 2 that are BLM Sensitive (Table 6). We detected the highest diversity of amphibians in the mixedgrass prairies of central South Dakota in close proximity to the Belle Fourche River. The least diverse area was in mixedgrass prairie in west central South Dakota (Appendix F). In the Butte County primary area we detected 3 species calling (Table F-1). In the Newell primary area we detected 1 species (Table F-2). In the Fort Meade primary area we detected 2 species (Table F-3). In the Mission Ridge primary area we detected 2 species calling (Table F-4). In the Two Rivers primary area we detected 4 species (Table F-5).

Across the study area we had 1,341 point observations of 6 amphibian species. We did not observe any amphibian species incidentally that were not detected during surveys. We had 62 incidental point observations of 8 reptile species (Table 6).
Table 6. Amphibian and reptile species detected within the survey area during structured surveys and incidentally. Columns display the number of calling survey points where each species was detected, the number of point observations of each species, and the proportion of primary areas within which each species was detected. Global ranks are listed, along with the state ranks of rare, threatened and endangered species. Species denoted with $a *$ are BLM Sensitive species.

\begin{tabular}{|c|c|c|c|c|}
\hline $\begin{array}{l}\text { Amphibian and } \\
\text { Reptile Species }\end{array}$ & 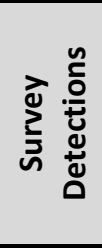 & 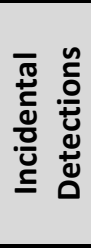 & 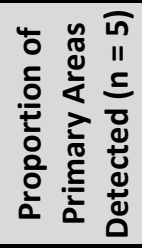 & 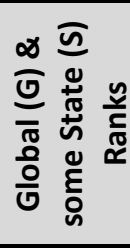 \\
\hline \multicolumn{5}{|l|}{ Amphibians } \\
\hline \multicolumn{5}{|l|}{ Mole Salamanders } \\
\hline $\begin{array}{l}\text { Western Tiger } \\
\text { Salamander }\end{array}$ & 0 & 9 & 0.5 & G5 \\
\hline \multicolumn{5}{|l|}{ True Toads } \\
\hline Great Plains Toad* & 19 & 3 & 0.25 & G5 \\
\hline Woodhouse's Toad & 13 & 9 & 0.25 & G5 \\
\hline \multicolumn{5}{|l|}{ True Frogs } \\
\hline $\begin{array}{l}\text { Northern Leopard } \\
\text { Frog }\end{array}$ & 10 & 31 & 0.75 & G5 \\
\hline Boreal Chorus Frog & 96 & 82 & 0.88 & G5 \\
\hline \multicolumn{5}{|l|}{ Spadefoot Toads } \\
\hline Plains Spadefoot* & 18 & 1 & 0.38 & G5 \\
\hline \multicolumn{5}{|l|}{ Reptiles } \\
\hline \multicolumn{5}{|l|}{ Pond Turtles } \\
\hline Painted Turtle & 0 & 12 & 0.63 & G5 \\
\hline \multicolumn{5}{|l|}{ Vipers } \\
\hline Prairie Rattlesnake & 0 & 2 & 0.13 & G5 \\
\hline \multicolumn{5}{|l|}{ Colubrid Snakes } \\
\hline Smooth Greensnake & 0 & 1 & 0.13 & S4 /G5 \\
\hline Gophersnake & 0 & 3 & 0.38 & G5 \\
\hline $\begin{array}{l}\text { Terrestrial } \\
\text { Gartersnake }\end{array}$ & 0 & 7 & 0.5 & G5 \\
\hline Plains Gartersnake & 0 & 11 & 0.38 & G5 \\
\hline \multicolumn{5}{|l|}{$\begin{array}{l}\text { Sagebrush/Spiny } \\
\text { Lizards }\end{array}$} \\
\hline $\begin{array}{l}\text { Greater Short-horned } \\
\text { Lizard* }\end{array}$ & 0 & 1 & 0.13 & S2 /G5 \\
\hline
\end{tabular}




\section{DisCUSSION/ RECOMMENDATIONS}

Over western South Dakota, riparian habitats within mixedgrass prairie on public lands generally lack shrub or tree cover (D. Bachen, personal observation). Both riparian and upland sites with adequate tree and shrub cover have been previously documented to support a diverse community of birds, bats, and terrestrial small mammals not typically found in associated prairie habitats (Bjugstad and Girard 1984, Finch and Ruggiero 1993). We similarly found that communities of bird and bat species differ between these habitat types (Tables C-1, $\mathrm{C}-2$, and $\mathrm{E}-2$ ). However, our ability to conduct surveys to further explore these community differences and document species occurring in riparian woodlands was hampered by a lack of riparian woodlands on public lands. We recommend future efforts focus on gaining access to existing riparian woodlands located on private property in order to conduct surveys in these habitats. We also recommend efforts to conserve existing riparian woodlands and restore riparian woodland areas that are becoming deforested by mimicking the natural frequency and intensity of disturbances resulting from historical grazing, fire, and flood regimes (Bjugstad and Girard 1984, Sieg 1995).

Naturally occurring and manmade lentic wetlands are biodiversity hotspots on the prairie landscape that are essential for some groups like waterbirds, amphibians, and aquatic reptiles. Use of wetlands by livestock has been shown to affect some species of wildlife through degradation of water quality and reduction of associated aquatic and terrestrial vegetation (Knutson et al. 2004, Schmutzer et al. 2008). We didn't see major impacts to wetland habitats during the course of our study in western South Dakota, but encourage managers to work toward maintaining wetlands in their present state by mimicking frequencies and intensities of disturbance associated with historical grazing regimes of native ungulates.

Terrestrial small mammals play an important role in many of the ecosystems within the study area. Small mammals are prey for a diversity of species including raptors and shrikes and their burrows also provide shelter for species like Burrowing Owls (Athene cunicularia) and Great Plains Toads (Anaxyrus cognatus). Both vegetation and soil type are important components of habitat for small mammals, and efforts to conserve these species should focus on these habitat attributes. One of the primary threats to small mammal populations is alteration of native habitat through the invasion of nonnative plant species which have the potential to alter the physical structure of vegetation and impact the abundance and distribution of these species (Litt and Pearson 2013, Bachen 2014). In some areas of the Butte County and Two Rivers primary areas, we found widespread dense stands of sweet clover (Melilotus officinalis). This invasive species appeared to be displacing native vegetation and may be affecting wildlife, although further research is necessary to confirm its effects on invaded ecosystems. Management efforts that mitigate the invasion of nonnative plants are likely to conserve existing small mammal populations and associated species.

Arguably some of the most valuable data we collected from this project are observations of bat species. Bat populations in general have not been studied as closely as other taxa, and can be negatively impacted by both development of wind energy (Kunz et al. 2007) 
and disease (Lorch et al. 2011). We found bat species within the study area to be most active during the warm season, with an apparent cessation of activity or migration out of the area between December and March. Winter bat roosts on the South Dakotas prairie habitats are likely absent or extremely rare. If they exist, identification and protection of those roosts is important. However, it is more likely that managers would be able to conserve bat species within this region by identifying and protecting summer roosts. Although general roost structures been documented for the species we observed (Kunz and Fenton 2006), little effort has been made to identify the specific types and importance of structures used in the Northern Great Plains, including South Dakota. Future efforts should be made to address this, including radio telemetry surveys and surveys of potential roost structures such as bridges and buildings. This and other information on bat species in this region would assist with mitigating potential impacts of wind energy development.

During this project we conducted visual encounter surveys of lentic waterbodies to detect breeding amphibians and aquatic reptiles, however due to logistical constraints we did not begin surveys until August, after some species had already metamorphosed and left breeding sites. Visual encounter surveys are valuable because they not only confirm breeding of amphibians, but unlike roadside calling surveys, they have the ability to detect amphibian species that either do not call or call softly as well as reptile species that use wetlands. Also by surveying during the breeding season for waterfowl and other water birds, surveys can provide valuable breeding records for these species. Therefore, we recommend future surveys starting in the late spring and early summer should be conducted to supplement the information we gathered with this project.

Prior to this effort, few baseline surveys for nongame species on BLM lands have been conducted and entered into the Montana Natural Heritage Program's databases. As past surveys documenting status and distribution of nongame species have likely been conducted in this area, further efforts should be made to incorporate existing structured survey data into the NHP database to increase availability of this data and aid in management of species across BLM managed lands in South Dakota.

This survey effort has increased the number of point observations in the Montana Natural Heritage Program's database by $40 \%$ to 4,122 ; increasing the number of observations for birds by $35 \%$, mammals by $61 \%$, amphibians by $2060 \%$, and reptiles by $77 \%$. Our surveys added 33 new bird species, including 4 that are BLM Sensitive: Brewers Sparrow (Spizella breweri), Burrowing Owl, Long-billed Curlew (Numenius americanus), and Sage Thrasher (Oreoscoptes montanus). This effort also add 6 mammal species, 4 amphibian species, including 2 South Dakota Rare, Threatened, or Endangered Animals (Great Plains Toad and Plains Spadefoot (Spea bombifrons) ), and 3 reptile species, including the Greater Short-horned Lizard (Phrynosoma hernandesi) which is a South Dakota Rare, Threatened, or Endangered Animal (SDNHP 2014). 


\section{LITERATURE Cited}

Bachen, D.A. 2014. Cheatgrass invasion of sagebrush steppe: impacts of vegetation structure on small mammals. Thesis, Montana State University, Bozeman, Montana, USA.

Backlund, D. C. 2002. The expanding distribution of the least shrew, Cryptotis parva, in South Dakota. Proceedings of the South Dakota Academy of Science. 81:153159.

Bjugstad, A. J., and M. Girard.1984. Wooded draws in rangelands of the northern Great Plains, guidelines for increasing wildlife on farms and ranches. Great Plains Agriculture Council and Kansas State University, Manhattan: 27B-36B.

Finch, D. M., and L. F. Ruggiero. 1993. Wildlife habitats and biological diversity in the Rocky Mountains and Northern Great Plains. Natural Areas Journal 13:191-203.

Foresman, K. 2012. Mammals of Montana. Second edition. Mountain Press Publishing Company, Missoula, Montana.

Heisler, L. M., C. M. Somers, T. I. Wellicome, and R. G. Poulin. 2013. Landscape-scale features affecting small mammal assemblages on the northern Great Plains of North America. Journal of Mammalogy 94:10591067.

Hendricks, P., S. Lenard, C. Currier, and B. Maxell. 2007. Filling the distribution gaps for small mammals in Montana. Montana Natural Heritage Program Technical Report. Helena, Montana.
Hendricks, P., S. Lenard, C. Currier, B. Maxell, and J. Carlson. 2008. Surveys for grassland birds of the Malta Field Office-BLM, including a seven-year study in north Valley County. Montana Natural Heritage Program Technical Report. Helena, Montana.

Heyer, W. R., M. A. Donnelly, and R. W. MacDiarmid. 1994. Measuring and monitoring biological diversity: Standard methods for amphibians. Biological Diversity Handbook Series. Smithsonian Institute Press, Washington DC, USA.

Knutson, M. G., W. B. Richardson, D. M. Reineke, B. R. Gray, J. R. Parmelee, and S. E. Weick. 2004. Agricultural ponds support amphibian populations. Ecological Applications 14:669-684.

Kunz, T.H. and M. B. Fenton editors. 2006. Bat Ecology. University of Chicago Press, Chicago, Illinois, USA.

Kunz, T. H., E. B. Arnett, W. P. Erickson, A. R. Hoar, G. D. Johnson, R. P. Larkin, M. D. Strickland, R. W. Thresher, and M. D. Tuttle. 2007. Ecological impacts of wind energy development on bats: questions, research needs, and hypotheses. Frontiers in Ecology and the Environment 5:315-324.

Litt, A. R., and D. E. Pearson. 2013. Non-native plants and wildlife in the Intermountain West. Wildlife Society Bulletin 37:517-526. 
Lorch, J. M., C. U. Meteyer, M. J. Behr, J. G. Boyles, P. M. Cryan, A. C. Hicks, A. E. Ballmann, J. T. H. Coleman, D. N. Redell, D. M. Reeder, and D. S. Blehert. 2011. Experimental infection of bats with Geomyces destructans causes white-nose syndrome. Nature 480:376-378.

(NOAA) National Oceanographic and Atmospheric Administration. 2012. 19812010 climate norms.

<http://www.ncdc.noaa.gov/oa/climate/nor mals/usnormals.html> Accessed 25 Oct 2014.

Natural Resources Conservation Service [NRCS], United States Department Of Agriculture [USDA]. Soil survey staff. Web soil survey. <http://websoilsurvey.nrcs.usda.gov/> Accessed 1 Nov 2014.

Pocock, M. J. O., and N. Jennings. 2006. Use of hair tubes to survey for shrews: new methods for identification and quantification of abundance. Mammal Review 36:299-308.
Schmutzer, A. C., M. J. Gray, E. C. Burton, and D. L. Miller. 2008. Impacts of cattle on amphibian larvae and the aquatic environment. Freshwater Biology 53:26132625.

Sieg, C. H. 1995. The role of fire in managing for biological diversity on native rangelands of the northern Great Plains. Conserving biodiversity on native rangelands: Symposium proceedings. USFS Gen. Tech. Rep. RM-GTR298.

(SDNHP) South Dakota Natural Heritage Program. 2014. Rare, threatened or endangered animals tracked by the South Dakota Natural Heritage Program.<http://gfp.sd.gov/wildlife/threaten ed-endangered/rare-animal.aspx $>$ Accessed November 2014.

Werner, J. K., B. A. Maxell, P. Hendricks, and D. L. Flath, editors. 2004. Amphibians and Reptiles of Montana. Mountain Press Publishing Company, Missoula, Montana. $262 \mathrm{p}$. 
APPENDIX A.

Global and State Rank Definition 


\section{Heritage Program Ranks}

The international network of Natural Heritage Programs employs a standardized ranking system to denote global (range-wide) and state status. Species are assigned numeric ranks ranging from 1 to 5 , reflecting the relative degree to which they are "at-risk". Rank definitions are given below. A number of factors are considered in assigning ranks - the number, size and distribution of known "occurrences" or populations, population trends (if known), habitat sensitivity, and threat. Factors in a species' life history that make it especially vulnerable are also considered (e.g., dependence on a specific pollinator).

Global RANK Definitions (NatureServe 2003)

G1 Critically imperiled because of extreme rarity and/or other factors making it highly vulnerable to extinction

G2 Imperiled because of rarity and/or other factors making it vulnerable to extinction

G3 Vulnerable because of rarity or restricted range and/or other factors, even though it maybe abundant at some of its locations

G4 Apparently secure, though it may be quite rare in parts of its range, especially at the periphery

G5 Demonstrably secure, though it may be quite rare in parts of its range, especially at the periphery

T1-5 Infraspecific Taxon (trinomial) - The status of infraspecific taxa (subspecies or varieties) are indicated by a "T-rank" following the species' global rank

\section{State Rank Definitions}

S1

S2

S3

S4

S5

At high risk because of extremely limited and potentially declining numbers, extent and/or habitat, making it highly vulnerable to extirpation in the state

At risk because of very limited and potentially declining numbers, extent and/or habitat, making it vulnerable to extirpation in the state

Potentially at risk because of limited and potentially declining numbers, extent and/or habitat, even though it may be abundant in some areas

Uncommon but not rare (although it may be rare in parts of its range), and usually widespread. Apparently not vulnerable in most of its range, but possibly cause for longterm concern

Common, widespread, and abundant (although it may be rare in parts of its range). Not vulnerable in most of its range 


\section{COMBINATION RANKS}

G\#G\# or S\#S\# Range Rank-A numeric range rank (e.g., G2G3) used to indicate uncertainty about the exact status of a taxon

\section{Qualifiers}

NR

Q

$\mathrm{X}$

$\mathrm{H}$

U

HYB

?

C

A

Z

$P$
Not ranked

Questionable taxonomy that may reduce conservation priority-Distinctiveness of this entity as a taxon at the current level is questionable; resolution of this uncertainty may result in change from a species to a subspecies or hybrid, or inclusion of this taxon in another taxon, with the resulting taxon having a lower-priority (numerically higher) conservation status rank

Presumed Extinct-Species believed to be extinct throughout its range. Not located despite intensive searches of historical sites and other appropriate habitat, and virtually no likelihood that it will be rediscovered

Possibly Extinct-Species known from only historical occurrences, but may never-the less still be extant; further searching needed

Unrankable-Species currently unrankable due to lack of information or due to substantially conflicting information about status or trends

Hybrid-Entity not ranked because it represents an interspecific hybrid and not a species

Inexact Numeric Rank-Denotes inexact numeric rank

Captive or Cultivated Only-Species at present is extant only in captivity or cultivation, or as a reintroduced population not yet established

Accidental-Species is accidental or casual in South Dakota, in other words, infrequent and outside usual range. Includes species (usually birds or butterflies) recorded once or only a few times at a location. A few of these species may have bred on the one or two occasions they were recorded

Zero Occurrences-Species is present but lacking practical conservation concern in South Dakota because there are no definable occurrences, although the taxon is native and appears regularly in South Dakota

Potential-Potential that species occurs in Montana but no extant or historic occurrences are accepted 
Reported-Species reported in South Dakota but without a basis for either accepting or rejecting the report, or the report not yet reviewed locally. Some of these are very recent discoveries for which the program has not yet received first-hand information; others are old, obscure reports

SYN Synonym - Species reported as occurring in South Dakota, but the South Dakota Natural Heritage Program does not recognize the taxon; therefore the species is not assigned a rank

* A rank has been assigned and is under review. Contact the South Dakota Natural Heritage Program for assigned rank

Breeding-Rank refers to the breeding population of the species in South Dakota

$\mathrm{N}$

Nonbreeding-Rank refers to the non-breeding population of the species in South Dakota 
APPENDIX B:

Echolocation Call Characteristics of Montana Bats 


\section{Echolocation Call Characteristics of Montana Bats ${ }^{1}$}

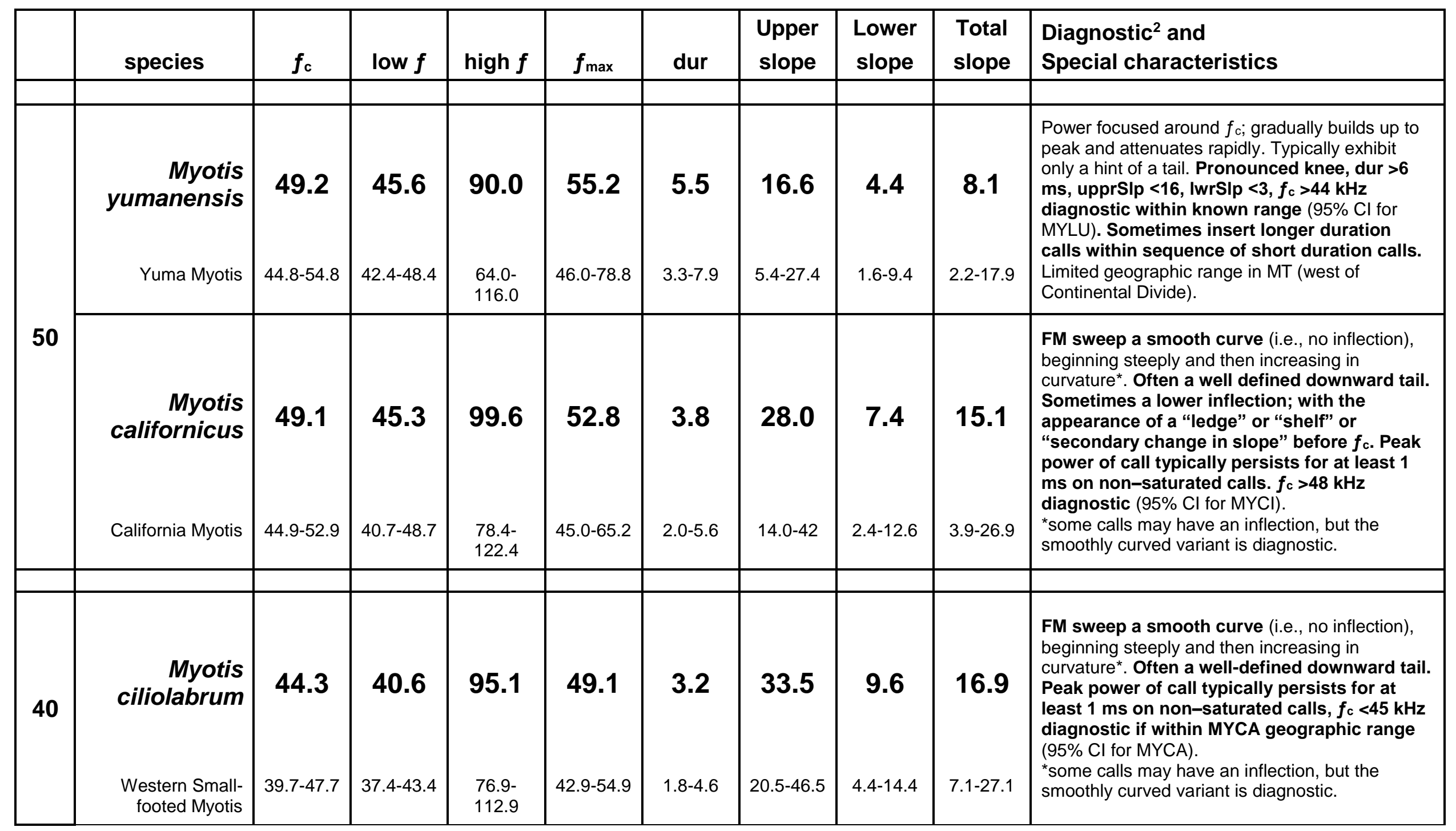




\begin{tabular}{|c|c|c|c|c|c|c|c|c|c|c|}
\hline & $\begin{array}{r}\text { Myotis } \\
\text { septentrionalis } \\
\text { Northern Long- } \\
\text { eared Myotis }\end{array}$ & $\begin{array}{c}43.2 \\
36.8-50.8\end{array}$ & $\begin{array}{c}37.0 \\
27.0-47.0\end{array}$ & $\begin{array}{l}86.0- \\
124.0\end{array}$ & $\begin{array}{c}51.3 \\
30.7-72.7\end{array}$ & $2.3-5.3$ & $\begin{array}{c}24.2 \\
11.8-35.8\end{array}$ & $\begin{array}{l}11.7 \\
3.1-20.3\end{array}$ & $\begin{array}{r}18.6 \\
9.4-29.4\end{array}$ & $\begin{array}{l}\text { Calls may have up to } \mathbf{1 0 0} \mathbf{~ k H z} \text { of bandwidth. } \\
\text { Shaped like MYEV or MYTH but distinguished by } \\
f_{c} \text {. FM sweep may be nearly linear making } f_{c} \\
\text { difficult to recognize. Quiet but consistent calls. } \\
\text { Presence in Montana uncertain - capture and } \\
\text { genetic analysis needed to confirm ID. }\end{array}$ \\
\hline & $\begin{array}{r}\text { Myotis } \\
\text { volans }\end{array}$ & $\begin{array}{c}41.6 \\
36.4-46.4\end{array}$ & $\begin{array}{c}36.9 \\
31.1-43.1\end{array}$ & $\begin{array}{l}89.6 \\
66.4- \\
112.4\end{array}$ & $\begin{array}{c}48.0 \\
39.0-60.0\end{array}$ & $\begin{array}{c}4.8 \\
2.4-7.0\end{array}$ & $\begin{array}{c}15.1 \\
6.9-22.9\end{array}$ & $\begin{array}{c}7.7 \\
1.1-14.3\end{array}$ & $\begin{array}{r}12.0 \\
4.0-22.0\end{array}$ & $\begin{array}{l}\text { May exhibit an upward sweep into the call; } \\
\text { uncommon, but diagnostic when present. } \\
\text { Generally has shorter, steeper calls than MYLU } \\
\text { in open (uncluttered) areas. Note that alias } \\
\text { harmonics may resemble upsweeps if sonogram } \\
\text { is truncated (e.g. } 96 \mathrm{kHz} \text { maximum). }\end{array}$ \\
\hline & $\begin{array}{r}\text { Myotis } \\
\text { Iucifugus }\end{array}$ & $\begin{array}{c}40.8 \\
37.2-43.2\end{array}$ & $\begin{array}{c}38.1 \\
33.9-41.9\end{array}$ & $\begin{array}{c}74.5 \\
51.5-97.5\end{array}$ & $\begin{array}{c}44.5 \\
36.0-53.5\end{array}$ & $\begin{array}{c}6.0 \\
3.2-8.6\end{array}$ & $\begin{array}{c}13.1 \\
2.7-26.9\end{array}$ & $\begin{array}{c}3.9 \\
0.8-9.1\end{array}$ & $\begin{array}{c}6.2 \\
1.6-13.8\end{array}$ & $\begin{array}{l}\text { Sometimes with multiple power centers making } \\
\text { calls look clumpy. Can make the longest duration } \\
\text { and lowest slope calls of all Myotis. Dur }>7 \mathrm{~ms} \\
\text { ( } 95 \% \mathrm{Cl} \text { for MYVO) and IwrSlp }<3 \text { diagnostic } \\
\text { among } 40 \mathrm{kHz} \text { Myotis; } f_{\mathrm{c}}<44 \mathrm{kHz} \text { diagnostic } \\
\text { west of Continental Divide ( } 95 \% \mathrm{Cl} \text { for MYYU). }\end{array}$ \\
\hline & $\begin{array}{r}\text { Lasiurus } \\
\text { borealis } \\
\text { Eastern Red Bat }\end{array}$ & $\begin{array}{c}40.4 \\
31.6-47.6\end{array}$ & $\begin{array}{c}40.2 \\
33.8-45.8\end{array}$ & $\begin{array}{c}\mathbf{6 7 . 6} \\
40.4-94.4\end{array}$ & $\begin{array}{c}43.8 \\
34.2-54.2\end{array}$ & $\begin{array}{c}6.8 \\
3.2-11.4\end{array}$ & $\begin{array}{l}10.0 \\
0.1-22\end{array}$ & $\begin{array}{c}2.0 \\
0.0-4.4\end{array}$ & $\begin{array}{r}4.4 \\
0.1-9.8\end{array}$ & $\begin{array}{l}\text { U-shaped calls; up-turn at end of call; may } \\
\text { exhibit variable } f_{\mathrm{c}} \text { across sequence. Power } \\
\text { smoothly centered in call. Typically } \sim 40 \mathrm{kHz} \text { calls } \\
\text { with dur }>10 \mathrm{~ms} \text { at LABO. }\end{array}$ \\
\hline \multirow{2}{*}{30} & $\begin{array}{l}\text { Myotis evotis } \\
\text { Long-eared Myotis }\end{array}$ & $\begin{array}{r}34.3 \\
31.7-37.7\end{array}$ & 28.1 & $\begin{array}{l}49.5- \\
107.5\end{array}$ & $\begin{array}{r}39.1 \\
31.0-46.9\end{array}$ & $\begin{array}{r}3.7 \\
2.1-5.3\end{array}$ & $\begin{array}{r}20.5 \\
6.1-35.5\end{array}$ & $\begin{array}{c}8.7 \\
2.3-15.3\end{array}$ & $\begin{array}{r}13.5 \\
4.9-24.5\end{array}$ & $\begin{array}{l}\text { Calls may have up to } 100 \mathrm{kHz} \text { of bandwidth. } \\
\text { Shaped like MYTH and MYSE but distinguished } \\
\text { by } f_{\mathrm{c}}=\mathbf{3 2 - 3 6} \text { (upper range boundary for MYTH, } \\
95 \% \text { Cls for MYVO and MYSE). FM sweep may } \\
\text { be nearly linear making } f_{\mathrm{c}} \text { difficult to recognize. } \\
\text { Harmonics converge toward primary call } \\
\text { component. }\end{array}$ \\
\hline & $\begin{array}{r}\text { Eptesicus } \\
\text { fuscus }\end{array}$ & 28.2 & $24.8-30.8$ & 43.4-69.4 & 31.9 & $2.8-12.2$ & $2.5-15.5$ & $0.3-4.3$ & $0.6-7.6$ & $\begin{array}{l}\text { Variable; calls with high } f \text { below } 60 \mathrm{kHz} \text { can be } \\
\text { confused with LANO. Calls with high } f>65 \mathrm{kHz} \\
\text { distinguish from LANO (high } f \text { range boundary } \\
\text { for LANO), }>12 \text { ms to distinguish from ANPA } \\
\text { where species coexist (duration range } \\
\text { boundary for ANPA); even long calls have some } \\
\text { FM component. Parallel harmonics. }\end{array}$ \\
\hline
\end{tabular}




\begin{tabular}{|c|c|c|c|c|c|c|c|c|c|c|}
\hline & $\begin{array}{r}\text { Antrozous } \\
\text { pallidus } \\
\text { Pallid Bat }\end{array}$ & $\begin{array}{r}28.0 \\
26.0-30.0\end{array}$ & $23.8-29.8$ & $41.5-67.5$ & $25.0-37.0$ & $3.8-10.0$ & $3.0-15.9$ & $0.6-5.1$ & $2.1-7.9$ & $\begin{array}{l}\text { Often simple curved FM sweep, sometimes with } \\
\text { knee in center. Distinguish from EPFU when }<6 \\
\text { calls/sec, but calculate this manually by } \\
\text { looking for call intervals }>180 \text { ms for } \geq 1 \\
\text { second. Note that MYTH \& MYEV can also be } \\
<6 \text { calls/sec. No tail. Parallel harmonics. } \\
\text { Presence of social calls diagnostic (see ref. } \\
\text { calls). }\end{array}$ \\
\hline & & & & & & & & & & \\
\hline \multirow{2}{*}{20} & $\begin{array}{r}\text { Lasionycteris } \\
\text { noctivagans } \\
\text { Silver-haired Bat }\end{array}$ & $25.5-27.5$ & $22.6-28.6$ & $26.0-58.5$ & $24.0-33.2$ & $2.3-16.8$ & $0.0-12.6$ & $0.0-3.7$ & $0.0-6.7$ & $\begin{array}{l}\text { Some call variants can be confused with EPFU. } \\
\text { Flat calls with } f_{\mathrm{c}} \geq 26 \mathbf{k H z} \text { diagnostic. Shorter } \\
\text { calls reverse J-shaped; often with a distinct } \\
\text { inflection. Parallel harmonics. Flat LACI calls are } \\
\text { lower in } f_{\mathrm{c}} \text {, but shorter LACI approach calls may } \\
\text { overlap short LANO calls (examine entire } \\
\text { sequence and call interval). Low slope calls with } \\
f_{\mathrm{c}}=25-26 \mathrm{kHz} \text { may be distinguished from LACI } \\
\text { by the presence of an inflection. EPFU has more } \\
\text { FM, typically with smooth curvature (no } \\
\text { inflection), and high } f>33 \mathrm{kHz} \text { (lower range } \\
\text { boundary). }\end{array}$ \\
\hline & $\begin{array}{r}\text { Myotis } \\
\text { thysanodes }\end{array}$ & 21.5-27.5 & $14.2-24.2$ & $\begin{array}{l}41.6- \\
103.6\end{array}$ & $24.0-39.3$ & $1.9-5.9$ & $7.1-33.0$ & $3.1-16.8$ & $4.9-24.1$ & $\begin{array}{l}\text { Calls may have up to } 100 \mathrm{kHz} \text { of bandwidth. } \\
\text { Shaped like MYEV but distinguished by } f_{\mathrm{c}} \text {. FM } \\
\text { sweep may be nearly linear making } f_{\mathrm{c}} \text { difficult to } \\
\text { recognize. Want to have presence of harmonics } \\
\text { to distinguish from COTO if high } f<50 \mathrm{kHz} \text {. } \\
\text { Continuous steep shape and } f_{\mathrm{c}} \text { down into the } \\
20 \text { s is diagnostic: totalSIp }>15, f_{\mathrm{c}}<28 \mathrm{kHz} \text {, } \\
\text { and low } f<24 \mathrm{kHz} \text { diagnostic or totalSIp } \\
>10, f_{\mathrm{c}}<28 \mathrm{kHz} \text {, and low } f<24 \mathrm{kHz} \text { diagnostic } \\
\text { if harmonics converge toward primary call } \\
\text { component. }\end{array}$ \\
\hline
\end{tabular}




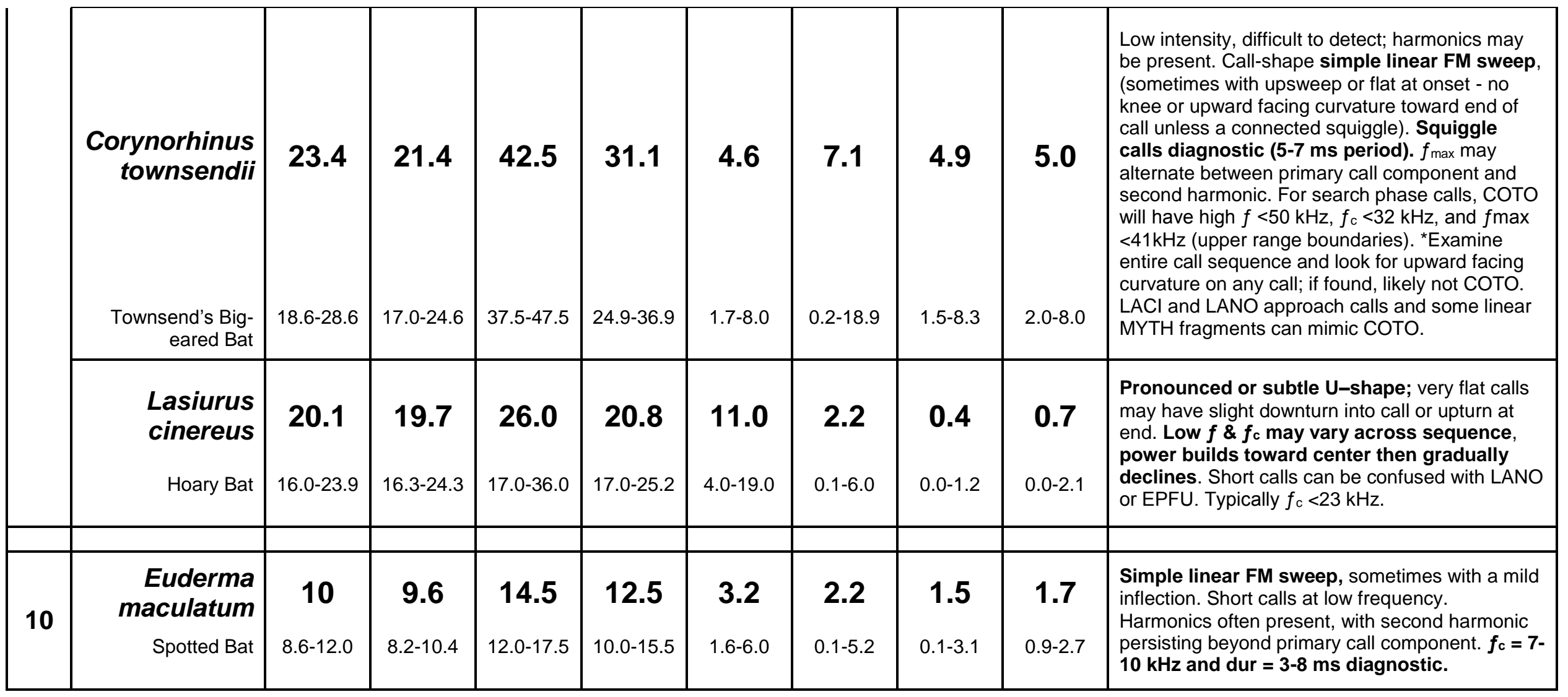

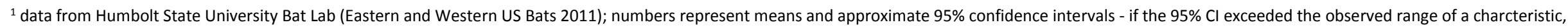
the range boundary was used.

${ }^{2}$ diagnostic characteristics for determination of species identification are bolded in text.

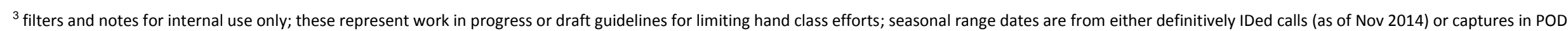
but are not set in stone. 
Primary call: the component of an echolocation sound emitted by a bat with the lowest frequency, also called the fundamental; typically the most powerful and sometimes the only part of the call visible on a sonogram

Harmonic: multiple, typically subtle components of the call, existing at higher frequencies but roughly parallel to the primary call component; presence may indicate higher call quality unless a call is oversaturated

The characteristics below refer to attributes of the primary call. In rare cases, a harmonic may be the most powerful component of a call; these characteristics and their corresponding values in this key are not applicable to those measured from a harmonic component.

low $f$ : lowest frequency $(\mathrm{kHz})$

high $f$ : highest frequency $(\mathrm{kHz})$

$f_{\mathrm{c}}$ : characteristic frequency, the frequency of the call at its lowest slope $(\mathrm{kHz})$

$f_{\text {max }}$ : the frequency where the power is greatest $(\mathrm{kHz})$

dur: duration (ms) from the start to the end of a call

Upper slope: the slope of the call $(\mathrm{kHz} / \mathrm{ms})$ between the high $f$ and the knee; abbreviated: upprSlp

Lower slope: the slope of the call $(\mathrm{kHz} / \mathrm{ms})$ between the knee and the $f_{c}$; abbreviated: IwrSlp

Total slope: the slope of the call $(\mathrm{kHz} / \mathrm{ms})$ between the high $f$ and the low $f$; abbreviated: totalSlp

Other terms used to describe calls:

FM: frequency modulation, change in frequency over time; most calls start at a high frequency and sweep down to a lower frequency

power: amplitude or sound energy (i.e. volume)

oversaturation: powerful calls may exceed the microphone/recorder capability and produce anomalies in the sonogram such as full spectrum "noise"

(clipping) or alias harmonics (upside-down harmonics); peak power duration cannot be accurately estimated

inflection or knee: pronounced change in slope; some calls may not have an obvious knee if very steep or smoothly curved

flat: a call or portion of a call with very low or no slope (horizontal), i.e. constant frequency (CF)

sequence: a series of bat calls, produced as a bat flies past the detector

calls/sec: the number of calls per second for a given period; note that Sonobat's calculation of this characteristic may be incorrect due to multiple bats in a recording, low intensity calls, and dead air space in a sequence - ms between calls should be examined and calls should be looked at in real time to accurately estimate this characteristic if needed

Note that all frequencies should be interpreted as apparent or observed frequencies. These values may vary from the frequency emitted by the bat due to distance to detector (decreasing call power or volume). Call volume may have a noticeable effect on all frequencies recorded depending on the location of the power in the call $(>5 \mathrm{kHz})$. 
The values for the characteristics listed in this key are based on search phase calls. Therefore, it is important to make sure that search phase calls are examined and analyzed during hand classification.

Search phase calls: used for general navigation and searching in uncluttered areas, generally consistent call characteristics, approximately 3-12 calls per second; bats may be able to detect objects $>10$ meters away with these calls ${ }^{3}$

Approach phase calls: used when approaching either prey or a landing site or in cluttered airspace, such as when flying around vegetation; these calls are typically steeper and shorter than search calls and frequencies may shift up significantly, often 10-25 calls per second

Feeding buzz: also called terminal phase calls, used for close proximity object location during prey pursuit/capture, may exceed 100 calls per second ${ }^{4}$; very steep and short calls that can mimic other species if interpreted as search calls, but can be much lower in volume/power; not useful for species ID

Social calls: used to communicate with other bats, often lower in frequency than search phase calls for a species and may contain complex frequency modulation patterns; may be very helpful for identifying some species (e.g. ANPA) but are irregularly recorded

\section{How to Use the Key for Montana Bats ${ }^{1}$}

Tip: Put bat detector in an open, uncluttered environment so that it is more likely to detect bats using search phase calls.

1. Look at search phase calls (not approach calls, feeding buzzes, or social calls) within a sequence.

2. Choose noise free calls with harmonics so that you are more likely to see the whole call instead of just a portion. Note that some calls may be oversaturated if the bat closely approached the microphone and these should be avoided if possible.

3. Look at the entire sequence in both compressed and real time views. This will help you see the whole picture (Are there multiple bats? Feeding buzzes or other non-search phase calls?). This is particularly important differentiating EPFU vs. ANPA, MYLU vs. LABO, and for COTO in general since many other species may have calls that mimic СОТО.

4. Look at the standard view for multiple calls within a sequence. BE AWARE that Sonobat sometimes identifies incorrect characteristics, analyzes strong harmonics instead of the primary call, and occasionally includes noise along with the primary call of interest.

${ }^{1}$ Adapted from Humbolt State University Bat Lab. 2011. Eastern and Western US Bat Keys.

${ }^{2}$ Reviewed in Fenton, M. B. 2013. Questions, ideas and tools: lessons from bat echolocation. Animal Behaviour 85, 869-879. Originally described in Griffin, D. R., et al. 1960. The echolocation of flying insects by bats. Animal Behaviour 8, 141-154.

${ }^{3}$ Fenton, M. B. 2004. Bat Natural History and Echolocation. In Brigham, R. M.,et al., eds. Bat Echolocation Research: tools, techniques, and analysis. Bat

Conservation International, Austin, TX.

${ }^{4}$ Elemans, C., et al. 2011. Superfast Muscles Set Maximum Call Rate in Echolocating Bats. Science 333, 1885-1888. 


\section{APPENDiX C:}

\section{BASEline Status Indices OF DETECTED SPECIES: BIRD SURVEYS}




\section{Butte County Primary Area}

Table C-1. Proportion of points where bird species were detected and average number (SE) of birds detected at those points in mixedgrass prairie habitats surveyed on driving transects. Species denoted by + are listed as BLM Sensitive. Standard errors could not be calculated for SE values denoted with a *.

\begin{tabular}{|c|c|c|}
\hline \multirow{2}{*}{ Species } & \multicolumn{2}{|c|}{ Mixedgrass Prairie $(n=48)$} \\
\hline & $\begin{array}{c}\text { Proportion of points } \\
\text { detected }\end{array}$ & Average number detected (SE) \\
\hline American Kestrel & 0.02 & $1(*)$ \\
\hline American Wigeon & 0.04 & $2(0)$ \\
\hline Barn Swallow & 0.31 & $1.87(1.81)$ \\
\hline Brown-Headed Cowbird & 0.56 & $2(1.52)$ \\
\hline Brewer's Blackbird & 0.02 & $1(*)$ \\
\hline Blue-Winged Teal & 0.06 & $1.67(0.58)$ \\
\hline Canada Goose & 0.29 & $1.43(0.65)$ \\
\hline Chestnut-Collared Longspur + & 0.4 & $2.47(1.61)$ \\
\hline Common Nighthawk & 0.02 & $1(*)$ \\
\hline Eastern Kingbird & 0.06 & $1.33(0.58)$ \\
\hline European Starling & 0.02 & $1(*)$ \\
\hline Great Blue Heron & 0.02 & $1(*)$ \\
\hline Golden Eagle + & 0.02 & $1\left(^{*}\right)$ \\
\hline Grasshopper Sparrow & 0.52 & $2.08(0.91)$ \\
\hline Horned Lark & 0.48 & $2.22(1.31)$ \\
\hline Killdeer & 0.35 & $1.24(0.44)$ \\
\hline Lark Bunting & 0.98 & $5.04(2.50)$ \\
\hline Lark Sparrow & 0.04 & $1(0)$ \\
\hline Mallard & 0.02 & $2(*)$ \\
\hline Mourning Dove & 0.04 & $1(0)$ \\
\hline Northern Harrier & 0.02 & $1(*)$ \\
\hline Red-Winged Blackbird & 0.46 & $2.45(1.65)$ \\
\hline Short-Eared Owl & 0.02 & $1(*)$ \\
\hline Spotted Sandpiper & 0.02 & $1\left(^{*}\right)$ \\
\hline Upland Sandpiper & 0.52 & $1.56(0.92)$ \\
\hline Vesper Sparrow & 0.58 & $1.57(0.79)$ \\
\hline Western Kingbird & 0.06 & $1.33(0.58)$ \\
\hline Western Meadowlark & 0.92 & $2.93(1.47)$ \\
\hline Wilson's Phalarope & 0.15 & $1.71(1.11)$ \\
\hline Yellow-Headed Blackbird & 0.02 & $2(*)$ \\
\hline
\end{tabular}


Table C-2. Proportion of points where bird species were detected and average number (SE) of birds detected at those points in riparian woodland habitats surveyed on walking transects. Species denoted by + are listed as BLM Sensitive. Standard errors could not be calculated for SE values denoted with a *.

\begin{tabular}{|c|c|c|}
\hline \multirow[b]{2}{*}{ Species } & \multicolumn{2}{|c|}{ Riparian Woodland $(n=13)$} \\
\hline & $\begin{array}{c}\text { Proportion of points } \\
\text { detected }\end{array}$ & Average number detected (SE) \\
\hline American Kestrel & 0.15 & $1(0)$ \\
\hline Bell's Vireo & 0.08 & $1(*)$ \\
\hline Brown-Headed Cowbird & 0.54 & $1.57(1.51)$ \\
\hline Black-Headed Grosbeak & 0.15 & $1(0)$ \\
\hline Brewer's Blackbird & 0.23 & $2.33(0.58)$ \\
\hline Brown Thrasher & 0.08 & $2(*)$ \\
\hline Cedar Waxwing & 0.15 & $1(0)$ \\
\hline Common Grackle & 0.23 & $3.33(2.52)$ \\
\hline Common Nighthawk & 0.08 & $1(*)$ \\
\hline Eastern Kingbird & 0.46 & $2.17(0.41)$ \\
\hline European Starling & 0.85 & $3.36(1.29)$ \\
\hline Hairy Woodpecker & 0.08 & $1(*)$ \\
\hline House Wren & 0.08 & $1(*)$ \\
\hline Killdeer & 0.23 & $1.67(1.15)$ \\
\hline Lark Bunting & 0.08 & $1\left(^{*}\right)$ \\
\hline Loggerhead Shrike + & 0.23 & $1.67(0.58)$ \\
\hline Mallard & 0.15 & $1(*)$ \\
\hline Mourning Dove & 0.92 & $3.17(2.00)$ \\
\hline Northern Flicker & 0.54 & $1.14(0.38)$ \\
\hline Orchard Oriole & 0.15 & $1(0)$ \\
\hline Red-Headed Woodpecker + & 0.08 & $1(*)$ \\
\hline Red-Tailed Hawk & 0.23 & $1(0)$ \\
\hline Red-Winged Blackbird & 0.77 & $2.8(1.75)$ \\
\hline Turkey Vulture & 0.08 & $1(*)$ \\
\hline Upland Sandpiper & 0.38 & $1.2(0.45)$ \\
\hline Vesper Sparrow & 0.15 & $1.5(0.71)$ \\
\hline Western Kingbird & 0.31 & $1.5(0.58)$ \\
\hline Western Meadowlark & 1 & $2.23(0.83)$ \\
\hline Willow Flycatcher & 0.08 & $1(*)$ \\
\hline Yellow Warbler & 0.31 & $1(0)$ \\
\hline
\end{tabular}




\section{Newell Primary Area}

Table C-3. Proportion of points where bird species were detected and average number (SE) of birds detected at those points in mixedgrass prairie habitats surveyed on driving transects. Species denoted by + are listed as BLM Sensitive. Standard errors could not be calculated for SE values denoted with a *.

\begin{tabular}{|c|c|c|}
\hline \multirow[b]{2}{*}{ Species } & \multicolumn{2}{|c|}{ Mixedgrass Prairie $(n=24)$} \\
\hline & $\begin{array}{c}\text { Proportion of points } \\
\text { detected }\end{array}$ & Average number detected (SE) \\
\hline Barn Swallow & 0.21 & $2(1.73)$ \\
\hline Brown-Headed Cowbird & 0.67 & $1.88(1.15)$ \\
\hline Canada Goose & 0.04 & $2(*)$ \\
\hline Chestnut-Collared Longspur + & 0.08 & $2(0)$ \\
\hline Common Grackle & 0.13 & $3.33(3.21)$ \\
\hline Common Nighthawk & 0.08 & $1(0)$ \\
\hline Eastern Kingbird & 0.13 & $1.33(0.58)$ \\
\hline European Starling & 0.17 & $3(1.41)$ \\
\hline Ferruginous Hawk + & 0.042 & $1(*)$ \\
\hline Golden Eagle + & 0.08 & $1(0)$ \\
\hline Grasshopper Sparrow & 0.67 & $2.31(1.14)$ \\
\hline Horned Lark & 0.63 & $2.47(1.41)$ \\
\hline Killdeer & 0.13 & $1.33(0.58)$ \\
\hline Lark Bunting & 0.96 & $6.91(3.27)$ \\
\hline Lark Sparrow & 0.13 & $1.33(0.58)$ \\
\hline Mallard & 0.08 & $1.5(0.71)$ \\
\hline Mourning Dove & 0.38 & $1.89(1.05)$ \\
\hline Northern Harrier & 0.04 & $1(*)$ \\
\hline $\begin{array}{l}\text { Northern Rough-Winged } \\
\text { Swallow }\end{array}$ & 0.13 & $2(0)$ \\
\hline Ring-Billed Gull & 0.04 & $1(*)$ \\
\hline Red-Winged Blackbird & 0.38 & $2.22(2.39)$ \\
\hline Sprague's Pipit + & 0.04 & $2(*)$ \\
\hline Swainson's Hawk & 0.08 & $1(0)$ \\
\hline Upland Sandpiper & 0.21 & $1.4(0.55)$ \\
\hline Western Kingbird & 0.17 & $1.25(0.5)$ \\
\hline Western Meadowlark & 1 & $4.5(1.67)$ \\
\hline Wilson's Phalarope & 0.04 & $1(*)$ \\
\hline Yellow-Headed Blackbird & 0.04 & $2(*)$ \\
\hline
\end{tabular}


Table C-4. Proportion of points where bird species were detected and average number (SE) of birds detected at those points in mixedgrass prairie habitats surveyed on walking transects. Species denoted by + are listed as BLM Sensitive. Standard errors could not be calculated for SE values denoted with a *.

\begin{tabular}{|l|c|c|}
\hline \multirow{2}{*}{ Species } & \multicolumn{2}{|c|}{ Mixedgrass Prairie ( $\mathbf{n}$ 29) } \\
\cline { 2 - 3 } & $\begin{array}{c}\text { Proportion of points } \\
\text { detected }\end{array}$ & Average number detected (SE) \\
\hline Brown-Headed Cowbird & 0.62 & $2.17(1.25)$ \\
\hline Bobolink + & 0.1 & $1(0)$ \\
\hline Cliff Swallow & 0.17 & $2(1.22)$ \\
\hline Common Nighthawk & 0.03 & $1\left(^{*}\right)$ \\
\hline Common Yellowthroat & 0.07 & $1(0)$ \\
\hline Eastern Kingbird & 0.07 & $1.5(0.71)$ \\
\hline European Starling & 0.03 & $0\left(^{*}\right)$ \\
\hline Grasshopper Sparrow & 0.72 & $2.86(1.15)$ \\
\hline Horned Lark & 0.55 & $2.94(2.32)$ \\
\hline Killdeer & 0.14 & $1.75(1.5)$ \\
\hline Lark Bunting & 0.79 & $4(2.73)$ \\
\hline Lark Sparrow & 0.03 & $2\left(^{*}\right)$ \\
\hline Long-Billed Curlew + & 0.03 & $1\left(^{*}\right)$ \\
\hline Loggerhead Shrike + & 0.03 & $1\left(^{*}\right)$ \\
\hline Mallard & 0.17 & $1.2(0.45)$ \\
\hline Merlin & 0.03 & $1\left(^{*}\right)$ \\
\hline Mourning Dove & 0.69 & $2.2(1.01)$ \\
\hline Ring-Billed Gull & 0.07 & $1(0)$ \\
\hline Ring-Necked Pheasant & 0.17 & $1.2(0.45)$ \\
\hline Red-Winged Blackbird & 0.31 & $1.44(0.73)$ \\
\hline Upland Sandpiper & 0.62 & $1.5(0.62)$ \\
\hline Western Meadowlark & 1 & $5.45(1.59)$ \\
\hline Wild Turkey & 0.14 & $1(0)$ \\
\hline Yellow Warbler & 0.03 & $2.0)$ \\
\hline Yellow-Headed Blackbird & 0.07 & \\
\hline & & \\
\hline
\end{tabular}




\section{Fort Meade Primary AREa}

Table C-5. Proportion of points where bird species were detected and average number (SE) of birds detected at those points in riparian woodland, mixedgrass prairie, and coniferous woodland habitats surveyed on driving transects. Species denoted by + are listed as BLM Sensitive. Standard errors could not be calculated for SE values denoted with a *

\begin{tabular}{|c|c|c|c|c|c|c|}
\hline \multirow[b]{2}{*}{ Species } & \multicolumn{2}{|c|}{ Riparian Woodland ( $n=5$ ) } & \multicolumn{2}{|c|}{ Mixedgrass Prairie $(n=12)$} & \multicolumn{2}{|c|}{ Coniferous Woodland $(n=7)$} \\
\hline & $\begin{array}{l}\text { Proportion } \\
\text { of points } \\
\text { detected }\end{array}$ & $\begin{array}{c}\text { Average } \\
\text { number } \\
\text { detected (SE) }\end{array}$ & $\begin{array}{l}\text { Proportion } \\
\text { of points } \\
\text { detected }\end{array}$ & $\begin{array}{c}\text { Average } \\
\text { number } \\
\text { detected (SE) }\end{array}$ & $\begin{array}{l}\text { Proportion } \\
\text { of points } \\
\text { detected }\end{array}$ & $\begin{array}{c}\text { Average } \\
\text { number } \\
\text { detected (SE) }\end{array}$ \\
\hline American Coot & 0 & 0 & 0.08 & $3(*)$ & 0 & 0 \\
\hline American Crow & 0.6 & $1(0)$ & 0.08 & $1(*)$ & 0.29 & $1.5(0.71)$ \\
\hline American White Pelican & 0.2 & $1(*)$ & 0 & 0 & 0.14 & $1(*)$ \\
\hline Barn Swallow & 0 & 0 & 0.33 & $1.25(0.5)$ & 0 & 0 \\
\hline Black-Capped Chickadee & 0.4 & $2(1.41)$ & 0.08 & $1(*)$ & 0.43 & $1.33(0.58)$ \\
\hline Black-Headed Grosbeak & 0.4 & $1(0)$ & 0 & 0 & 0.14 & $1(*)$ \\
\hline Brewer's Blackbird & 0.2 & $4(*)$ & 0.25 & $1(0)$ & 0.14 & $1(*)$ \\
\hline Brown-Headed Cowbird & 0 & 0 & 0.5 & $2.83(3.06)$ & 0.71 & $1.6(0.89)$ \\
\hline Canada Goose & 0 & 0 & 0.08 & $1(*)$ & 0 & 0 \\
\hline Chipping Sparrow & 0.2 & $1(*)$ & 0 & 0 & 0.29 & $1.5(0.71)$ \\
\hline Common Grackle & 0 & 0 & 0.08 & $1\left(^{*}\right)$ & 0.29 & $1(0)$ \\
\hline Dark-Eyed Junco & 0 & 0 & 0 & 0 & 0.14 & $1(*)$ \\
\hline Dusky Flycatcher & 0.2 & $1(*)$ & 0 & 0 & 0 & 0 \\
\hline Eastern Kingbird & 0 & 0 & 0.25 & $1(0)$ & 0.14 & $2(*)$ \\
\hline Loggerhead Shrike + & 0 & 0 & 0.08 & $2(*)$ & 0 & 0 \\
\hline Mallard & 0 & 0 & 0.08 & $1\left(^{*}\right)$ & 0 & 0 \\
\hline Mountain Bluebird & 0 & 0 & 0 & 0 & 0.14 & $1(*)$ \\
\hline Mourning Dove & 0.4 & $1.5(0.71)$ & 0.5 & $2.17(1.17)$ & 0.29 & $1(0)$ \\
\hline Northern Flicker & 0.2 & $1(*)$ & 0 & 0 & 0.14 & $1(*)$ \\
\hline Plumbeous Vireo & 0 & 0 & 0 & 0 & 0.14 & $1\left(^{*}\right)$ \\
\hline Ring-Billed Gull & 0 & 0 & 0.17 & $2.5(0.71)$ & 0 & 0 \\
\hline Red-Breasted Nuthatch & 0.2 & $1(*)$ & 0.08 & $1(*)$ & 0.43 & $1.33(0.58)$ \\
\hline Red-Eyed Vireo & 0.2 & $1(*)$ & 0 & 0 & 0 & 0 \\
\hline
\end{tabular}




\begin{tabular}{|l|c|c|c|c|c|c|}
\hline \multirow{2}{*}{ Species } & \multicolumn{2}{|c|}{ Riparian Woodland $(\mathbf{n}=\mathbf{5})$} & \multicolumn{2}{c|}{ Mixedgrass Prairie ( $\mathbf{n = 1 2 )}$} & \multicolumn{2}{c|}{ Coniferous Woodland (n= 7) } \\
\cline { 2 - 7 } & $\begin{array}{c}\text { Proportion } \\
\text { of points } \\
\text { detected }\end{array}$ & $\begin{array}{c}\text { Average } \\
\text { number } \\
\text { detected (SE) }\end{array}$ & $\begin{array}{c}\text { Proportion } \\
\text { of points } \\
\text { detected }\end{array}$ & $\begin{array}{c}\text { Average } \\
\text { number } \\
\text { detected (SE) }\end{array}$ & $\begin{array}{c}\text { Proportion } \\
\text { of points } \\
\text { detected }\end{array}$ & $\begin{array}{c}\text { Average } \\
\text { number } \\
\text { detected (SE) }\end{array}$ \\
\hline Red-Winged Blackbird & 0 & 0 & 0.75 & $3.56(2.4)$ & 0 & 0 \\
\hline Rock Pigeon & 0 & 0 & 0 & 0 & 0.14 & $2\left(^{*}\right)$ \\
\hline Spotted Towhee & 0 & 0 & 0 & 0 & 0.43 & $2.67(1.53)$ \\
\hline Tree Swallow & 0.4 & $4(2.83)$ & 0.08 & $2\left(^{*}\right)$ & 0.14 & $2\left(^{*}\right)$ \\
\hline Turkey Vulture & 0 & 0 & 0.17 & $1(0)$ & 0 & 0 \\
\hline Upland Sandpiper & 0 & 0 & 0.08 & $1\left(^{*}\right)$ & 0 & 0 \\
\hline Vesper Sparrow & 0 & 0 & 0.08 & $1\left(^{*}\right)$ & 0 & 0 \\
\hline Western Meadowlark & 0.2 & $1\left(^{*}\right)$ & 0.92 & $2.64(1.21)$ & 0.43 & $3.33(2.08)$ \\
\hline Western Tanager & 0.4 & $1.5(0.71)$ & 0 & 0 & 0 & 0 \\
\hline Western Wood-Pewee & 0 & 0 & 0.08 & $1\left(^{*}\right)$ & 0 & 0 \\
\hline White-Breasted Nuthatch & 0 & 0 & 0 & 0 & 0.14 & $1\left(^{*}\right)$ \\
\hline Wild Turkey & 0.2 & $1\left(^{*}\right)$ & 0 & 0 & 0.14 & $1\left(^{*}\right)$ \\
\hline Wilson's Snipe & 0 & 0 & 0.17 & $1.5(0.71)$ & 0 & 0 \\
\hline Yellow Warbler & 0.2 & $4\left(^{*}\right)$ & 0 & 0 & 0 & 0 \\
\hline
\end{tabular}




\section{Lead Primary Area}

Table C-6. Proportion of points where bird species were detected and average number (SE) of birds detected at those points in coniferous woodland habitats surveyed on walking transects. Species denoted by + are listed as BLM Sensitive. Standard errors could not be calculated for SE values denoted with a *

\begin{tabular}{|c|c|c|}
\hline \multirow[b]{2}{*}{ Species } & \multicolumn{2}{|c|}{ Coniferous Woodland $(n=10)$} \\
\hline & $\begin{array}{c}\text { Proportion of points } \\
\text { detected }\end{array}$ & Average number detected (SE) \\
\hline American Crow & 0.1 & $1(*)$ \\
\hline American Robin & 0.8 & $1.88(0.83)$ \\
\hline Black-Capped Chickadee & 0.5 & $1.4(0.89)$ \\
\hline Cedar Waxwing & 0.1 & $1\left(^{*}\right)$ \\
\hline Common Yellowthroat & 0.1 & $1(*)$ \\
\hline Dark-Eyed Junco & 0.2 & $1(0)$ \\
\hline Ovenbird & 0.3 & $1(0)$ \\
\hline Red-Breasted Nuthatch & 0.6 & $1.33(0.52)$ \\
\hline Ruby-Crowned Kinglet & 0.9 & $2.22(1.30)$ \\
\hline Red-Naped Sapsucker & 0.2 & $1(0)$ \\
\hline Song Sparrow & 0.4 & $1.25(0.5)$ \\
\hline Spotted Towhee & 0.1 & $1(*)$ \\
\hline Swainson's Thrush & 0.2 & $1(0)$ \\
\hline Tree Swallow & 0.1 & $1(*)$ \\
\hline Yellow Warbler & 0.2 & $2(1.41)$ \\
\hline Yellow-Rumped Warbler & 0.5 & $1.6(0.55)$ \\
\hline
\end{tabular}




\section{Southern Black Hills Primary Area}

Table C-7. Proportion of points where bird species were detected and average number (SE) of birds detected at those points in coniferous woodland, mixedgrass prairie, and riparian woodland habitats surveyed on driving transects. Species denoted by + are listed as BLM Sensitive. Standard errors could not be calculated for SE values denoted with a *.

\begin{tabular}{|c|c|c|c|c|c|c|}
\hline \multirow[b]{2}{*}{ Species } & \multicolumn{2}{|c|}{ Coniferous Woodland $(n=6)$} & \multicolumn{2}{|c|}{ Mixedgrass Prairie $(n=5)$} & \multicolumn{2}{|c|}{ Riparian Woodland $(n=1)$} \\
\hline & $\begin{array}{l}\text { Proportion } \\
\text { of points } \\
\text { detected }\end{array}$ & $\begin{array}{c}\text { Average } \\
\text { number } \\
\text { detected (SE) }\end{array}$ & $\begin{array}{l}\text { Proportion } \\
\text { of points } \\
\text { detected }\end{array}$ & $\begin{array}{c}\text { Average } \\
\text { number } \\
\text { detected (SE) }\end{array}$ & $\begin{array}{l}\text { Proportion } \\
\text { of points } \\
\text { detected }\end{array}$ & $\begin{array}{c}\text { Average } \\
\text { number } \\
\text { detected (SE) }\end{array}$ \\
\hline American Crow & 0.17 & $1(*)$ & 0.2 & $1(*)$ & 0 & 0 \\
\hline American Goldfinch & 0 & 0 & 0.2 & $1(*)$ & 0 & 0 \\
\hline American Robin & 0.33 & $1.5(0.71)$ & 0.2 & $1(*)$ & 0 & 0 \\
\hline $\begin{array}{l}\text { Black-Capped } \\
\text { Chickadee }\end{array}$ & 0.33 & $1(0)$ & 0 & 0 & 0 & 0 \\
\hline $\begin{array}{l}\text { Brown-Headed } \\
\text { Cowbird }\end{array}$ & 0.33 & $1(0)$ & 0.4 & $1(0)$ & 0 & 0 \\
\hline Cedar Waxwing & 0.17 & $4(*)$ & 0 & 0 & 0 & 0 \\
\hline Chipping Sparrow & 0.5 & $1.67(1.15)$ & 0.2 & $1\left(^{*}\right)$ & 0 & 0 \\
\hline Common Nighthawk & 0.17 & $1(*)$ & 0 & 0 & 0 & 0 \\
\hline Eastern Kingbird & 0 & 0 & 0.2 & $1(*)$ & 0 & 0 \\
\hline European Starling & 0 & 0 & 0 & 0 & 1 & $2(*)$ \\
\hline Grasshopper Sparrow & 0 & 0 & 0.6 & $1.33(0.58)$ & 0 & 0 \\
\hline House Wren & 0.17 & $1(*)$ & 0.2 & $1(*)$ & 0 & 0 \\
\hline Lark Bunting & 0 & 0 & 0.4 & $1.5(0.71)$ & 1 & $2(*)$ \\
\hline Lark Sparrow & 0.67 & $1.75(1.5)$ & 0.2 & $1(*)$ & 0 & 0 \\
\hline Lazuli Bunting & 0 & 0 & 0.2 & $1(*)$ & 0 & 0 \\
\hline Mountain Bluebird & 0 & 0 & 0.4 & $1.5(0.71)$ & 0 & 0 \\
\hline Mourning Dove & 0.83 & $3.4(1.67)$ & 0.8 & $6.25(5.68)$ & 0 & 0 \\
\hline Northern Flicker & 0.17 & $1(*)$ & 0 & 0 & 0 & 0 \\
\hline Rock Wren & 0.33 & $3(0)$ & 0.2 & $1(*)$ & 0 & 0 \\
\hline Red-Winged Blackbird & 0.17 & $3(*)$ & 0.2 & $1(*)$ & 1 & $1(*)$ \\
\hline Spotted Towhee & 0.83 & $1.6(0.89)$ & 0.6 & $1.67(1.15)$ & 0 & 0 \\
\hline Upland Sandpiper & 0.17 & $1\left(^{*}\right)$ & 0 & 0 & 0 & 0 \\
\hline Vesper Sparrow & 0.33 & $1(0)$ & 0 & 0 & 0 & 0 \\
\hline Western Meadowlark & 1 & $2.83(1.17)$ & 1 & $3.2(1.48)$ & 1 & $5\left(^{*}\right)$ \\
\hline Yellow Warbler & 0 & 0 & 0 & 0 & 1 & $1\left(^{*}\right)$ \\
\hline
\end{tabular}


Table C-8. Proportion of points where bird species were detected and average number (SE) of birds detected at those points in coniferous woodland, mixedgrass prairie, and riparian woodland habitats surveyed on walking transects. Species denoted by + are listed as BLM Sensitive. Standard errors could not be calculated for SE values denoted with a *.

\begin{tabular}{|c|c|c|c|c|c|c|}
\hline \multirow[b]{2}{*}{ Species } & \multicolumn{2}{|c|}{ Coniferous Woodland $(n=14)$} & \multicolumn{2}{|c|}{ Mixedgrass Prairie $(n=6)$} & \multicolumn{2}{|c|}{ Riparian Woodland $(n=1)$} \\
\hline & $\begin{array}{l}\text { Proportion of } \\
\text { points } \\
\text { detected }\end{array}$ & $\begin{array}{c}\text { Average } \\
\text { number } \\
\text { detected (SE) }\end{array}$ & $\begin{array}{l}\text { Proportion } \\
\text { of points } \\
\text { detected }\end{array}$ & $\begin{array}{c}\text { Average } \\
\text { number } \\
\text { detected (SE) }\end{array}$ & $\begin{array}{l}\text { Proportion } \\
\text { of points } \\
\text { detected }\end{array}$ & $\begin{array}{c}\text { Average } \\
\text { number } \\
\text { detected } \\
\text { (SE) }\end{array}$ \\
\hline American Crow & 0.07 & $2(*)$ & 0.17 & $1(*)$ & 0 & 0 \\
\hline American Goldfinch & 0.07 & $1(*)$ & 0 & 0 & 0 & 0 \\
\hline American Robin & 0.5 & $1.71(0.95)$ & 0.33 & $1(0)$ & 1 & $2(*)$ \\
\hline American White Pelican & 0.07 & $1(*)$ & 0 & 0 & 1 & $1(*)$ \\
\hline $\begin{array}{l}\text { Black-Capped } \\
\text { Chickadee }\end{array}$ & 0.36 & $1.6(0.55)$ & 0.17 & $1\left(^{*}\right)$ & 0 & 0 \\
\hline Brown-Headed Cowbird & 0.36 & $2(1.41)$ & 0.33 & $2(1.41)$ & 0 & 0 \\
\hline Chipping Sparrow & 0.64 & $2.22(0.94)$ & 0.33 & $1.5(0.71)$ & 0 & 0 \\
\hline Common Nighthawk & 0 & 0 & 0.17 & $1(*)$ & 0 & 0 \\
\hline Eastern Kingbird & 0.14 & $1(0)$ & 0 & 0 & 0 & 0 \\
\hline Field Sparrow & 0.07 & $1(*)$ & 0 & 0 & 0 & 0 \\
\hline Gray Catbird & 0.07 & $1(*)$ & 0 & 0 & 0 & 0 \\
\hline Grasshopper Sparrow & 0.07 & $1\left(^{*}\right)$ & 0.5 & $1.67(0.58)$ & 0 & 0 \\
\hline House Wren & 0.36 & $2.22(0.45)$ & 0.17 & $1(*)$ & 0 & 0 \\
\hline Horned Lark & 0 & 0 & 0.17 & $3(*)$ & 0 & 0 \\
\hline Lark Sparrow & 0.64 & $2.22(0.97)$ & 0.5 & $1.33(0.58)$ & 0 & 0 \\
\hline Lazuli Bunting & 0 & 0 & 0.17 & $1(*)$ & 0 & 0 \\
\hline Mountain Bluebird & 0.07 & $3(*)$ & 0.17 & $1(*)$ & 0 & 0 \\
\hline Mourning Dove & 0.57 & $2.13(0.99)$ & 0.67 & $2.75(0.96)$ & 1 & $3(*)$ \\
\hline Northern Flicker & 0.21 & $1(0)$ & 0.17 & $1(*)$ & 0 & 0 \\
\hline Ovenbird & 0.07 & $1(*)$ & 0 & 0 & 0 & 0 \\
\hline Red-Breasted Nuthatch & 0.07 & $1(*)$ & 0 & 0 & 0 & 0 \\
\hline Red-Eyed Vireo & 0 & 0 & 0.17 & $1(*)$ & 0 & 0 \\
\hline Rock Wren & 0.07 & $1(*)$ & 0 & 0 & 0 & 0 \\
\hline Song Sparrow & 0.21 & $1.67(0.58)$ & 0 & 0 & 0 & 0 \\
\hline Spotted Towhee & 0.71 & $1.5(0.97)$ & 0.17 & $1(*)$ & 1 & $2(*)$ \\
\hline Tree Swallow & 0.21 & $1(0)$ & 0 & 0 & 0 & 0 \\
\hline Upland Sandpiper & 0 & 0 & 0.17 & $2(*)$ & 0 & 0 \\
\hline Vesper Sparrow & 0.14 & $1.5(0.71)$ & 0.33 & $1.5(0.71)$ & 0 & 0 \\
\hline Western Meadowlark & 0.71 & $2.6(1.58)$ & 1 & $2.5(1.05)$ & 0 & 0 \\
\hline Western Tanager & 0.07 & $1(*)$ & 0.17 & $1(*)$ & 0 & 0 \\
\hline Willow Flycatcher & 0.07 & $1(*)$ & 0 & 0 & 0 & 0 \\
\hline Yellow Warbler & 0.21 & $1(0)$ & 0.17 & $2(*)$ & 1 & $2(*)$ \\
\hline
\end{tabular}




\section{Pedro Primary Area}

Table C-9. Proportion of points where bird species were detected and average number (SE) of birds detected at those points in mixedgrass prairie, riparian woodland, and coniferous woodland habitats surveyed on driving transects. Species denoted by + are listed as BLM Sensitive. Standard errors could not be calculated for SE values denoted with a *.

\begin{tabular}{|c|c|c|c|c|c|c|}
\hline \multirow[b]{2}{*}{ Species } & \multicolumn{2}{|c|}{ Mixedgrass Prairie $(n=8)$} & \multicolumn{2}{|c|}{ Riparian Woodland $(n=3)$} & \multicolumn{2}{|c|}{ Coniferous Woodland $(n=5)$} \\
\hline & $\begin{array}{l}\text { Proportion } \\
\text { of points } \\
\text { detected }\end{array}$ & $\begin{array}{c}\text { Average } \\
\text { number } \\
\text { detected (SE) }\end{array}$ & $\begin{array}{l}\text { Proportion } \\
\text { of points } \\
\text { detected }\end{array}$ & $\begin{array}{c}\text { Average } \\
\text { number } \\
\text { detected (SE) }\end{array}$ & $\begin{array}{l}\text { Proportion of } \\
\text { points } \\
\text { detected }\end{array}$ & $\begin{array}{c}\text { Average } \\
\text { number } \\
\text { detected (SE) }\end{array}$ \\
\hline American Crow & 0.5 & $1.5(1)$ & 0.67 & $1(0)$ & 0.4 & $1(0)$ \\
\hline American Kestrel & 0.13 & $1(*)$ & 0.33 & $1\left(^{*}\right)$ & 0 & 0 \\
\hline Bald Eagle + & 0.13 & $1(*)$ & 0 & 0 & 0 & 0 \\
\hline Barn Swallow & 0.38 & $3(2.65)$ & 0.33 & $1(*)$ & 0 & 0 \\
\hline Black-Billed Magpie & 0.25 & $1(0)$ & 0 & 0 & 0.2 & $1\left(^{*}\right)$ \\
\hline Brown-Headed Cowbird & 0.75 & $5(7.43)$ & 0.67 & $1(0)$ & 1 & $1.4(0.55)$ \\
\hline Blue Grosbeak & 0.25 & $1(0)$ & 0 & 0 & 0.4 & $2(1.41)$ \\
\hline American White Pelican & 0 & 0 & 0.33 & $1\left(^{*}\right)$ & 0 & 0 \\
\hline Bobolink & 0.13 & $1(*)$ & 0.33 & $1\left(^{*}\right)$ & 0 & 0 \\
\hline Burrowing Owl + & 0.13 & $1(*)$ & 0 & 0 & 0 & 0 \\
\hline Chipping Sparrow & 0 & 0 & 0 & 0 & 0.6 & $2(0)$ \\
\hline Common Grackle & 0.38 & $1.67(0.58)$ & 0.33 & $1\left(^{*}\right)$ & 0.2 & $1\left(^{*}\right)$ \\
\hline Common Yellowthroat & 0 & 0 & 0.33 & $1\left(^{*}\right)$ & 0 & 0 \\
\hline Dickcissel & 0.25 & $2(0)$ & 0.67 & $4(0)$ & 0 & 0 \\
\hline European Starling & 0.13 & $4(*)$ & 0.33 & $1(*)$ & 0 & 0 \\
\hline Field Sparrow & 0 & 0 & 0 & 0 & 0.2 & $3(*)$ \\
\hline Great Blue Heron & 0.25 & $1(0)$ & 0 & 0 & 0.2 & $2(*)$ \\
\hline Golden Eagle + & 0.13 & $1\left(^{*}\right)$ & 0 & 0 & 0 & 0 \\
\hline Grasshopper Sparrow & 0.13 & $1\left(^{*}\right)$ & 0.33 & $2(*)$ & 0.2 & $1\left(^{*}\right)$ \\
\hline Horned Lark & 0.13 & $6\left(^{*}\right)$ & 0 & 0 & 0 & 0 \\
\hline House Wren & 0.25 & $1(0)$ & 0 & 0 & 0.2 & $3(*)$ \\
\hline Lark Bunting & 0.13 & $1\left(^{*}\right)$ & 0 & 0 & 0 & 0 \\
\hline Lark Sparrow & 0.13 & $2(*)$ & 0.67 & $2(1.41)$ & 0.6 & $2(1.73)$ \\
\hline Mourning Dove & 1 & $3.88(1.96)$ & 1 & $5.33(1.53)$ & 1 & $2.4(1.14)$ \\
\hline Northern Flicker & 0.13 & $1(*)$ & 0.33 & $1\left(^{*}\right)$ & 0.2 & $1\left(^{*}\right)$ \\
\hline N. Rough-Winged & 0 & 0 & 0 & 0 & 0.2 & $2(*)$ \\
\hline Ring-Necked Pheasant & 0.5 & $1(0)$ & 0.67 & $1(0)$ & 0 & 0 \\
\hline Red-Winged Blackbird & 0.88 & $2.14(1.46)$ & 0.33 & $1(*)$ & 0.2 & $1(*)$ \\
\hline Spotted Towhee & 0.13 & $1(*)$ & 0 & 0 & 0.8 & $2(0.82)$ \\
\hline Tree Swallow & 0.25 & $1(0)$ & 0.33 & $2\left(^{*}\right)$ & 0 & 0 \\
\hline Turkey Vulture & 0.13 & $1\left(^{*}\right)$ & 0 & 0 & 0 & 0 \\
\hline Upland Sandpiper & 0.5 & $1(0)$ & 0.33 & $2(*)$ & 0.2 & $1\left(^{*}\right)$ \\
\hline Western Kingbird & 0.13 & $2(*)$ & 0 & 0 & 0.2 & $1(*)$ \\
\hline Western Meadowlark & 1 & $2.88(0.64)$ & 1 & $4(*)$ & 1 & $2.8(1.3)$ \\
\hline Wild Turkey & 0.25 & $1.5(0.71)$ & 0 & 0 & 0 & 0 \\
\hline Yellow Warbler & 0 & 0 & 0.33 & $1\left(^{*}\right)$ & 0 & 0 \\
\hline
\end{tabular}


Table C-10. Proportion of points where bird species were detected and average number (SE) of birds detected at those points in mixedgrass prairie and coniferous woodland habitats surveyed on walking transects. Species denoted by + are listed as BLM Sensitive. Standard errors could not be calculated for SE values denoted with a *

\begin{tabular}{|c|c|c|c|c|}
\hline \multirow[b]{2}{*}{ Species } & \multicolumn{2}{|c|}{ Mixedgrass Prairie $(n=7)$} & \multicolumn{2}{|c|}{ Coniferous Woodland $(n=23)$} \\
\hline & $\begin{array}{l}\text { Proportion of } \\
\text { points detected }\end{array}$ & $\begin{array}{c}\text { Average number } \\
\text { detected (SE) }\end{array}$ & $\begin{array}{l}\text { Proportion of } \\
\text { points detected }\end{array}$ & $\begin{array}{l}\text { Average number } \\
\text { detected (SE) }\end{array}$ \\
\hline American Crow & 0.71 & $1.6(0.89)$ & 0.57 & 1.230769 \\
\hline American Goldfinch & 0 & 0 & 0.26 & $2(1.55)$ \\
\hline American Robin & 0 & 0 & 0.09 & $1(0)$ \\
\hline American White Pelican & 0.14 & $2(*)$ & 0 & 0 \\
\hline Black-Billed Magpie & 0.29 & $1.5(0.71)$ & 0.39 & $1.44(0.53)$ \\
\hline Black-Capped Chickadee & 0.14 & $1(*)$ & 0.3 & $1.29(0.49)$ \\
\hline Blue Grosbeak & 0.43 & $1.33(0.58)$ & 0 & 0 \\
\hline Blue Jay & 0 & 0 & 0.04 & $1\left(^{*}\right)$ \\
\hline Brown-Headed Cowbird & 0.57 & $1.25(0.5)$ & 0.91 & $3.33(3.51)$ \\
\hline Brown Thrasher & 0 & 0 & 0.13 & $1.33(0.58)$ \\
\hline Cedar Waxwing & 0 & 0 & 0.09 & $1.5(0.71)$ \\
\hline Chipping Sparrow & 0.14 & $1(*)$ & 0.09 & $1(0)$ \\
\hline Common Grackle & 0.14 & $1(*)$ & 0.04 & $2(*)$ \\
\hline Eastern Kingbird & 0.14 & $1(*)$ & 0 & 0 \\
\hline Field Sparrow & 0.86 & $1.33(0.82)$ & 0.74 & $1.94(1.09)$ \\
\hline Golden Eagle + & 0 & 0 & 0.09 & $1(0)$ \\
\hline Grasshopper Sparrow & 0.14 & $1(*)$ & 0 & 0 \\
\hline Great Blue Heron & 0 & 0 & 0.04 & $2(*)$ \\
\hline House Wren & 0 & 0 & 0.09 & $1.5(0.71)$ \\
\hline Killdeer & 0.14 & $2(*)$ & 0 & 0 \\
\hline Lark Sparrow & 0.86 & $3.67(1.97)$ & 0.09 & $2.5(1.54)$ \\
\hline Long-Billed Curlew + & 0.14 & $1(*)$ & 0 & 0 \\
\hline Merlin & 0 & 0 & 0.04 & $1\left(^{*}\right)$ \\
\hline Mourning Dove & 0.86 & $4.83(3.06)$ & 0.96 & $2.91(1.69)$ \\
\hline Northern Flicker & 0.14 & $4(*)$ & 0.04 & $1(*)$ \\
\hline Northern Rough-Winged & 0.14 & $2(*)$ & 0.22 & $2.2(1.64)$ \\
\hline Orchard Oriole & 0 & 0 & 0.04 & $2(*)$ \\
\hline Ring-Necked Pheasant & 0.14 & $1(*)$ & 0.09 & $1(0)$ \\
\hline Red-Winged Blackbird & 0.14 & $1\left(^{*}\right)$ & 0 & 0 \\
\hline Rock Wren & 0 & 0 & 0.09 & $1(0)$ \\
\hline Spotted Towhee & 0.29 & $1.5(0.71)$ & 0.74 & $1.82(0.73)$ \\
\hline Turkey Vulture & 0 & 0 & 0.04 & $3(*)$ \\
\hline Upland Sandpiper & 0 & 0 & 0.09 & $1(0)$ \\
\hline Western Kingbird & 0 & 0 & 0.04 & $1(*)$ \\
\hline Western Meadowlark & 0.71 & $2.4(0.89)$ & 0.39 & $1.67(0.71)$ \\
\hline Yellow-Breasted Chat & 0 & 0 & 0.13 & $1.33(0.58)$ \\
\hline Yellow Warbler & 0 & 0 & 0.09 & $1.5(0.71)$ \\
\hline
\end{tabular}




\section{Mission Ridge Primary Area}

Table C-11. Proportion of points where bird species were detected and average number (SE) of birds detected at those points in mixedgrass prairie and riparian woodland habitats surveyed on driving transects. Species denoted by + are listed as BLM Sensitive. Standard errors could not be calculated for SE values denoted with a *.

\begin{tabular}{|c|c|c|c|c|}
\hline \multirow[b]{2}{*}{ Species } & \multicolumn{2}{|c|}{ Mixedgrass Prairie $(n=23)$} & \multicolumn{2}{|c|}{ Riparian Woodland $(n=1)$} \\
\hline & $\begin{array}{l}\text { Proportion of } \\
\text { points detected }\end{array}$ & $\begin{array}{c}\text { Average number } \\
\text { detected (SE) }\end{array}$ & $\begin{array}{l}\text { Proportion of } \\
\text { points detected }\end{array}$ & $\begin{array}{l}\text { Average number } \\
\text { detected (SE) }\end{array}$ \\
\hline Barn Swallow & 0.13 & $1.67(1.15)$ & 1 & $1(*)$ \\
\hline Bell's Vireo & 0 & 0 & 1 & $1\left(^{*}\right)$ \\
\hline Bobolink & 0.17 & $1.5(0.58)$ & 0 & 0 \\
\hline $\begin{array}{l}\text { Brown-headed } \\
\text { Cowbird }\end{array}$ & 0.91 & $3.81(2.86)$ & 0 & 0 \\
\hline Brown Thrasher & 0 & 0 & 1 & $3(*)$ \\
\hline Burrowing Owl + & 0.04 & $1(*)$ & 0 & 0 \\
\hline Common Grackle & 0.13 & $1.67(0.58)$ & 0 & 0 \\
\hline Common Nighthawk & 0.04 & $1(*)$ & 1 & $1(*)$ \\
\hline Dickcissel & 0.65 & $2.2(1.37)$ & 1 & $1(*)$ \\
\hline Eastern Kingbird & 0.04 & $1(*)$ & 1 & $1\left(^{*}\right)$ \\
\hline Gadwall & 0.04 & $2(*)$ & 0 & 0 \\
\hline Great Blue Heron & 0.04 & $1\left(^{*}\right)$ & 0 & 0 \\
\hline Grasshopper Sparrow & 0.43 & $1.6(0.70)$ & 0 & 0 \\
\hline Horned Lark & 0.43 & $1.8(1.23)$ & 0 & 0 \\
\hline Killdeer & 0.09 & $1(0)$ & 0 & 0 \\
\hline Lark Bunting & 0.96 & $4.01(2.29)$ & 0 & 0 \\
\hline Long-Billed Curlew + & 0.09 & $1(0)$ & 0 & 0 \\
\hline Mallard & 0.26 & $2.67(2.73)$ & 1 & $2(*)$ \\
\hline Mourning Dove & 0.43 & $2.8(2.86)$ & 1 & $2(*)$ \\
\hline Northern Pintail & 0.04 & $1\left(^{*}\right)$ & 0 & 0 \\
\hline Orchard Oriole & 0.04 & $1\left(^{*}\right)$ & 0 & 0 \\
\hline Ring-Necked Pheasant & 0.35 & $1.25(0.46)$ & 1 & $1\left(^{*}\right)$ \\
\hline Red-Winged Blackbird & 0.74 & $2.65(1.93)$ & 1 & $12(*)$ \\
\hline Spotted Towhee & 0 & 0 & 1 & $1\left(^{*}\right)$ \\
\hline Upland Sandpiper & 0.48 & $1.36(0.92)$ & 1 & $2(*)$ \\
\hline Western Kingbird & 0.04 & $1\left(^{*}\right)$ & 0 & 0 \\
\hline Western Meadowlark & 0.96 & $3.41(1.56)$ & 0 & 0 \\
\hline Yellow Warbler & 0 & 0 & 1 & $1\left(^{*}\right)$ \\
\hline
\end{tabular}




\section{TWo RIVERS PRIMARY AREA}

Table C-12. Proportion of points where bird species were detected and average number (SE) of birds detected at those points in coniferous woodland, badlands, riparian woodland, and mixedgrass prairie habitats surveyed on walking transects. Species denoted by + are listed as BLM Sensitive. Standard errors could not be calculated for SE values denoted with a *.

\begin{tabular}{|c|c|c|c|c|c|c|c|c|}
\hline \multirow[b]{2}{*}{ Species } & \multicolumn{2}{|c|}{$\begin{array}{l}\text { Coniferous Woodland } \\
\qquad(n=14)\end{array}$} & \multicolumn{2}{|c|}{$\begin{array}{l}\text { Badlands } \\
(n=10)\end{array}$} & \multicolumn{2}{|c|}{$\begin{array}{l}\text { Riparian Woodland } \\
\qquad(\mathrm{n}=1)\end{array}$} & \multicolumn{2}{|c|}{$\begin{array}{l}\text { Mixedgrass Prairie } \\
\qquad(n=2)\end{array}$} \\
\hline & $\begin{array}{l}\text { Proportion } \\
\text { of points } \\
\text { detected }\end{array}$ & $\begin{array}{c}\text { Average } \\
\text { number } \\
\text { detected } \\
\text { (SE) }\end{array}$ & $\begin{array}{l}\text { Proportion } \\
\text { of points } \\
\text { detected }\end{array}$ & $\begin{array}{c}\text { Average } \\
\text { number } \\
\text { detected } \\
\text { (SE) }\end{array}$ & $\begin{array}{c}\text { Proportion } \\
\text { of points } \\
\text { detected }\end{array}$ & $\begin{array}{c}\text { Average } \\
\text { number } \\
\text { detected } \\
\text { (SE) }\end{array}$ & $\begin{array}{l}\text { Proportion } \\
\text { of points } \\
\text { detected }\end{array}$ & $\begin{array}{c}\text { Average } \\
\text { number } \\
\text { detected } \\
\text { (SE) }\end{array}$ \\
\hline American Crow & 0.29 & $1(0)$ & 0.2 & $1.5(0.71)$ & 0 & 0 & 0 & 0 \\
\hline American Goldfinch & 0.14 & $1.5(0.71)$ & 0 & 0 & 0 & 0 & 0 & 0 \\
\hline American Kestrel & 0.07 & $1\left(^{*}\right)$ & 0.1 & $1(*)$ & 0 & 0 & 0 & 0 \\
\hline American Robin & 0 & 0 & 0.1 & $1\left(^{*}\right)$ & 0 & 0 & 0 & 0 \\
\hline Black-Billed Magpie & 0.14 & $1.5(0.71)$ & 0.4 & $2(0.82)$ & 1 & $1(*)$ & 1 & $4(2.83)$ \\
\hline Blue-Gray Gnatcatcher + & 0.07 & $1\left(^{*}\right)$ & 0 & 0 & 0 & 0 & 0 & 0 \\
\hline Brown-Headed Cowbird & 0.71 & $3(1.56)$ & 0.7 & $4.86(4.95)$ & 1 & $3(*)$ & 0.5 & $2(*)$ \\
\hline Blue Grosbeak & 0.36 & $1.4(0.89)$ & 0.1 & $1(*)$ & 0 & 0 & 0 & 0 \\
\hline Blue Jay & 0.07 & $1\left(^{*}\right)$ & 0 & 0 & 0 & 0 & 0 & 0 \\
\hline Brown Thrasher & 0.07 & $1(*)$ & 0 & 0 & 0 & 0 & 0 & 0 \\
\hline Canyon Wren & 0.07 & $1\left(^{*}\right)$ & 0 & 0 & 0 & 0 & 0 & 0 \\
\hline Cedar Waxwing & 0.07 & $7(*)$ & 0.1 & $3(*)$ & 0 & 0 & 0 & 0 \\
\hline Chipping Sparrow & 0.07 & $1\left(^{*}\right)$ & 0 & 0 & 0 & 0 & 0 & 0 \\
\hline Common Nighthawk & 0 & 0 & 0.1 & $1(*)$ & 0 & 0 & 0.5 & $1(*)$ \\
\hline Eastern Bluebird & 0.07 & $1(*)$ & 0 & 0 & 0 & 0 & 0 & 0 \\
\hline Eastern Kingbird & 0.07 & $1(*)$ & 0.1 & $1(*)$ & 0 & 0 & 0 & 0 \\
\hline Field Sparrow & 0.86 & $1.83(0.72)$ & 0.8 & $1.38(0.52)$ & 1 & $1\left(^{*}\right)$ & 1 & $1(0)$ \\
\hline Grasshopper Sparrow & 0 & 0 & 0 & 0 & 0 & 0 & 1 & $2(0)$ \\
\hline Horned Lark & 0 & 0 & 0.1 & $1(*)$ & 0 & 0 & 0 & 0 \\
\hline Killdeer & 0.07 & $1(*)$ & 0.1 & $1(*)$ & 0 & 0 & 0 & 0 \\
\hline Lark Bunting & 0.14 & $1(0)$ & 0.2 & $13(15.56)$ & 0 & 0 & 0 & 0 \\
\hline Lark Sparrow & 1 & $2.71(1.38)$ & 1 & $2.2(1.03)$ & 0 & 0 & 0.5 & $1\left(^{*}\right)$ \\
\hline Mourning Dove & 0.93 & $2.23(1.48)$ & 1 & $2.3(0.95)$ & 1 & $2(*)$ & 1 & $1.5(0.71)$ \\
\hline $\begin{array}{l}\text { Northern Rough- } \\
\text { Winged Swallow }\end{array}$ & 0.21 & $2.33(1.53)$ & 0.7 & $1.29(0.76)$ & 0 & 0 & 0.5 & $3(*)$ \\
\hline Rock Wren & 0.29 & $1.75(0.5)$ & 0.1 & $1(*)$ & 0 & 0 & 0.5 & $2(*)$ \\
\hline Red-Winged Blackbird & 0.07 & $4(*)$ & 0 & 0 & 0 & 0 & 0 & 0 \\
\hline Spotted Towhee & 0.5 & $1.86(1.07)$ & 0.4 & $1.25(0.5)$ & 1 & $1(*)$ & 1 & $1(0)$ \\
\hline Upland Sandpiper & 0.14 & $1(0)$ & 0 & 0 & 1 & $2(*)$ & 0.5 & $1(*)$ \\
\hline Western Kingbird & 0 & 0 & 0.1 & $1\left(^{*}\right)$ & 0 & 0 & 0 & 0 \\
\hline Western Meadowlark & 1 & $2.71(1.20)$ & 0.9 & $3.67(1.5)$ & 1 & $6(*)$ & 1 & $3.5(0.71)$ \\
\hline Yellow-Breasted Chat & 0 & 0 & 0.1 & $1\left(^{*}\right)$ & 0 & 0 & 0 & 0 \\
\hline
\end{tabular}


APPENDIX D

Baseline Status Indices of Detected Species: Small Mammal SuRVeys 


\section{Butte County Primary ARea}

Table D-1. Proportion of traplines each species was captured on within mixedgrass prairie, barren, herbaceous wetland, and shrubland habitats.

\begin{tabular}{|c|c|c|c|c|}
\hline Species & Mixedgrass Prairie $(n=6)$ & Barren $(n=1)$ & Herbaceous Wetland $(n=1)$ & Shrubland $(n=3)$ \\
\hline Shrew Spp. & 0 & 0 & 0 & 0.33 \\
\hline Thirteen-lined Ground Squirrel & 0.17 & 0 & 0 & 0.33 \\
\hline Meadow Vole & 0 & 0 & 1 & 0 \\
\hline Northern Grasshopper Mouse & 0.17 & 0 & 0 & 0.33 \\
\hline Deer Mouse & 0.33 & 1 & 0 & 1 \\
\hline Western Harvest Mouse & 0 & 0 & 0 & 0.33 \\
\hline
\end{tabular}

Table D-2. Proportion of museum special (MS), Sherman (SH), Victor (V), and pitfall (P) traps where small mammal species were detected within mixedgrass prairie, barren, herbaceous wetland, and shrubland habitats. Sample sizes of traps are shown in parentheses.

\begin{tabular}{|c|c|c|c|c|c|c|c|c|c|c|c|c|c|c|c|c|}
\hline \multirow[b]{2}{*}{ Species } & \multicolumn{4}{|c|}{ Mixedgrass Prairie $(n=6)$} & \multicolumn{4}{|c|}{ Barren (n = 1) } & \multicolumn{4}{|c|}{ Herbaceous Wetland ( $n=1)$} & \multicolumn{4}{|c|}{ Shrubland (n=3) } \\
\hline & $\begin{array}{c}\text { MS } \\
(n=164) \\
\end{array}$ & $\begin{array}{c}\mathrm{SH} \\
(\mathrm{n}=177) \\
\end{array}$ & $\begin{array}{c}V \\
(n=168) \\
\end{array}$ & $\begin{array}{c}P \\
(n=180) \\
\end{array}$ & $\begin{array}{c}\text { MS } \\
(n=28) \\
\end{array}$ & $\begin{array}{c}\mathrm{SH} \\
(\mathrm{n}=29) \\
\end{array}$ & $\begin{array}{c}V \\
(n=27)\end{array}$ & $\begin{array}{c}P \\
(n=30) \\
\end{array}$ & $\begin{array}{c}\text { MS } \\
(n=26) \\
\end{array}$ & $\begin{array}{c}\mathrm{SH} \\
(\mathrm{n}=30)\end{array}$ & $\begin{array}{c}V \\
(n=27) \\
\end{array}$ & $\begin{array}{c}P \\
(n=29) \\
\end{array}$ & $\begin{array}{c}\text { MS } \\
(n=75) \\
\end{array}$ & $\begin{array}{c}\mathrm{SH} \\
(\mathrm{n}=87) \\
\end{array}$ & $\begin{array}{c}V \\
(n=86) \\
\end{array}$ & $\begin{array}{c}P \\
(n=90) \\
\end{array}$ \\
\hline Deer Mouse & 0.04 & $<0.01$ & 0.04 & 0 & 0.07 & 0 & 0 & 0 & 0 & 0 & 0 & 0 & 0.08 & 0.01 & 0.06 & 0 \\
\hline Meadow vole & 0 & 0 & 0 & 0 & 0 & 0 & 0 & 0 & 0.04 & 0 & 0 & 0 & 0 & 0 & 0 & 0 \\
\hline $\begin{array}{c}\text { Northern } \\
\text { Grasshopper } \\
\text { Mouse }\end{array}$ & $<0.01$ & 0 & 0 & 0 & 0 & 0 & 0 & 0 & 0 & 0 & 0 & 0 & 0.01 & 0 & 0 & 0 \\
\hline $\begin{array}{c}\text { Thirteen- } \\
\text { lined Ground } \\
\text { Squirrel } \\
\end{array}$ & $<0.01$ & 0 & 0 & 0 & 0 & 0 & 0 & 0 & 0 & 0 & 0 & 0 & 0.01 & 0 & 0 & 0 \\
\hline Shrew spp. & 0 & 0 & 0 & 0 & 0 & 0 & 0 & 0 & 0 & 0 & 0 & 0 & 0 & 0 & 0 & 0.01 \\
\hline $\begin{array}{l}\text { Western } \\
\text { Harvest } \\
\text { Mouse } \\
\end{array}$ & 0 & 0 & 0 & 0 & 0 & 0 & 0 & 0 & 0 & 0 & 0 & 0 & 0 & 0.01 & 0 & 0 \\
\hline
\end{tabular}




\section{NeWell Primary ARea}

Table D-3. Proportion of traplines each species was captured on within mixedgrass prairie habitat.

\begin{tabular}{|c|c|}
\hline Species & Mixedgrass Prairie $(\mathbf{n}=\mathbf{6})$ \\
\hline Deer Mouse & 1 \\
\hline Western Harvest Mouse & 0.17 \\
\hline Plains Harvest Mouse & 0.17 \\
\hline
\end{tabular}

Table D-4. Proportion of museum special (MS), Sherman (SH), Victor (V), and pitfall (P) traps where small mammal species were detected within mixedgrass prairie habitat. Sample sizes of traps are shown in parentheses.

\begin{tabular}{|c|c|c|c|c|}
\hline \multirow[b]{2}{*}{ Species } & \multicolumn{4}{|c|}{ Mixedgrass Prairie $(n=6)$} \\
\hline & $\underset{\substack{\text { MS } \\
(n)}}{\text { MS7 }}$ & $\begin{array}{c}\mathrm{SH} \\
(n=152)\end{array}$ & $\begin{array}{c}V \\
(n=151)\end{array}$ & $\underset{(n=158)}{P}$ \\
\hline Deer Mouse & 0.07 & 0.01 & 0.01 & 0 \\
\hline Western Harvest Mouse & 0.01 & 0 & 0 & 0 \\
\hline Plains Harvest Mouse & 0 & 0 & 0.01 & 0 \\
\hline
\end{tabular}




\section{Fort Meade Primary Area}

Table D-5. Proportion of traplines each species was captured on within mixedgrass prairie, herbaceous wetland, deciduous woodland, and coniferous woodland habitats.

\begin{tabular}{|c|c|c|c|c|}
\hline Species & Mixedgrass Prairie $(n=1)$ & Herbaceous Wetland $(n=1)$ & Deciduous Woodland $(n=2)$ & Coniferous Woodland $(n=1)$ \\
\hline Deer Mouse & 1 & 1 & 1 & 1 \\
\hline White-footed Mouse & 0 & 0 & 1 & 1 \\
\hline Prairie Vole & 1 & 1 & 0.5 & 0 \\
\hline Meadow Vole & 0 & 1 & 0.5 & 0 \\
\hline
\end{tabular}

Table D-6. Proportion of museum special (MS), Sherman (SH), Victor (V), and pitfall (P) traps where small mammal species were detected within mixedgrass prairie, herbaceous wetland, deciduous woodland, and coniferous woodland habitats. Sample sizes of traps are shown in parentheses.

\begin{tabular}{|c|c|c|c|c|c|c|c|c|c|c|c|c|c|c|c|c|}
\hline \multirow{2}{*}{ Species } & \multicolumn{4}{|c|}{ Mixedgrass Prairie $(n=1)$} & \multicolumn{4}{|c|}{ Herbaceous Wetland $(n=1)$} & \multicolumn{4}{|c|}{ Deciduous Woodland $(n=2)$} & \multicolumn{4}{|c|}{ Coniferous Woodland $(n=1)$} \\
\hline & $\underset{(n=22)}{M S}$ & $\begin{array}{c}\text { SH } \\
(n=29)\end{array}$ & $\underset{(n=28)}{V}$ & $\underset{(n=30)}{P}$ & $\begin{array}{c}\text { MS } \\
(n=24)\end{array}$ & $\begin{array}{c}\text { SH } \\
(n=26)\end{array}$ & $\underset{(n=25)}{V}$ & $\underset{(n=29)}{P}$ & $\begin{array}{c}\text { MS } \\
(n=48)\end{array}$ & $\begin{array}{c}\text { SH } \\
(n=56)\end{array}$ & $\underset{(n=48)}{V}$ & $\underset{(n=60)}{P}$ & $\underset{(n=22)}{M S}$ & $\begin{array}{c}\text { SH } \\
(n=27)\end{array}$ & $\begin{array}{c}V \\
(n=25)\end{array}$ & $\underset{(n=30)}{P}$ \\
\hline Deer Mouse & 0.09 & 0.14 & 0.11 & 0 & 0.08 & 0 & 0 & 0 & 0.12 & 0.02 & 0.04 & 0 & 0 & 0 & 0.04 & 0 \\
\hline White-footed Mouse & 0 & 0 & 0 & 0 & 0 & 0 & 0 & 0 & 0.27 & 0.11 & 0.12 & 0 & 0.09 & 0 & 0 & 0 \\
\hline Prairie Vole & 0.41 & 0.03 & 0.07 & 0.03 & 0.04 & 0.04 & 0 & 0 & 0.02 & 0 & 0 & 0 & 0 & 0 & 0 & 0 \\
\hline Meadow Vole & 0 & 0 & 0 & 0 & 0.04 & 0.04 & 0 & 0.07 & 0 & 0.02 & 0 & 0 & 0 & 0 & 0 & 0 \\
\hline
\end{tabular}




\section{LeAd Primary AREA}

Table D-7 Proportion of traplines each species was captured on within coniferous woodland, wet meadow, and woody wetland habitats.

\begin{tabular}{|c|c|c|c|}
\hline Species & Coniferous Woodland $(\mathbf{n}=\mathbf{3})$ & Wet Meadow $(\mathbf{n}=1)$ & Woody Wetland $(\mathbf{n}=\mathbf{1})$ \\
\hline Masked Shrew & 0.33 & 0 & 1 \\
\hline Red-backed Vole & 1 & 0 & 0 \\
\hline Long-tailed Vole & 0.67 & 1 & 1 \\
\hline Meadow Vole & 0.67 & 0 & 1 \\
\hline Deer Mouse & 0.67 & 0 & 1 \\
\hline White-footed Mouse & 0 & 0 & 0 \\
\hline
\end{tabular}

Table D-8. Proportion of museum special (MS), Sherman (SH), Victor (V), and pitfall (P) traps where small mammal species were detected within coniferous woodland, wet meadow, and woody wetland habitats. Sample sizes of traps are shown in parentheses.

\begin{tabular}{|c|c|c|c|c|c|c|c|c|c|c|c|c|}
\hline \multirow[b]{2}{*}{ Species } & \multicolumn{4}{|c|}{ Coniferous Woodland $(n=3)$} & \multicolumn{4}{|c|}{ Wet Meadow $(n=1)$} & \multicolumn{4}{|c|}{ Woody Wetland ( $n=1)$} \\
\hline & $\begin{array}{c}\text { MS } \\
(n=82)\end{array}$ & $\begin{array}{c}\mathrm{SH} \\
(\mathrm{n}=86)\end{array}$ & $\begin{array}{c}\mathbf{V} \\
(n=85)\end{array}$ & $\begin{array}{c}P \\
(n=90)\end{array}$ & $\begin{array}{c}\text { MS } \\
(n=24)\end{array}$ & $\begin{array}{c}\mathrm{SH} \\
(\mathrm{n}=30)\end{array}$ & $\begin{array}{c}V \\
(n=26)\end{array}$ & $\begin{array}{c}P \\
(n=30)\end{array}$ & $\begin{array}{c}\text { MS } \\
(n=27)\end{array}$ & $\begin{array}{c}\mathrm{SH} \\
(\mathrm{n}=30)\end{array}$ & $\begin{array}{c}V \\
(n=30)\end{array}$ & $\begin{array}{c}P \\
(n=30)\end{array}$ \\
\hline Masked Shrew & 0 & 0 & 0.01 & 0 & 0.04 & 0 & 0 & 0.07 & 0.07 & & 0.03 & 0 \\
\hline Red-backed Vole & 0.09 & 0.03 & 0.04 & 0 & 0 & 0 & 0 & 0 & 0 & 0 & 0 & 0 \\
\hline Long-tailed Vole & 0.04 & 0 & 0.04 & 0 & 0 & 0 & 0 & 0 & 0 & 0 & 0 & 0 \\
\hline Meadow Vole & 0.02 & 0 & 0 & 0 & 0.12 & 0 & 0.08 & 0 & 0.04 & 0.03 & 0.07 & 0 \\
\hline Deer Mouse & 0.04 & 0.01 & 0.01 & 0 & 0 & 0 & 0 & 0 & 0.19 & 0.10 & 0.03 & 0 \\
\hline White-footed Mouse & 0 & 0 & 0 & 0 & 0 & 0 & 0 & 0 & 0.04 & 0 & 0 & 0 \\
\hline Least Chipmunk & 0 & 0 & 0.01 & 0 & 0 & 0 & 0 & 0 & 0 & 0 & 0 & 0 \\
\hline
\end{tabular}




\section{Southern Black Hills Primary Area}

Table D-9. Proportion of traplines each species was captured on within mixedgrass prairie and coniferous woodland habitats.

\begin{tabular}{|c|c|c|}
\hline Species & Mixedgrass Prairie $(\mathbf{n}=\mathbf{3})$ & Coniferous Woodland $(\mathbf{n}=\mathbf{2})$ \\
\hline Hispid Pocket Mouse & 0.66 & 0.5 \\
\hline Prairie Vole & 0.33 & 1 \\
\hline Deer Mouse & 0 & 0 \\
\hline Plains Harvest Mouse & 0.33 & 0.5 \\
\hline
\end{tabular}

Table D-10. Proportion of museum special (MS), Sherman (SH), Victor (V), and pitfall (P) traps where small mammal species were detected within mixedgrass prairie and coniferous woodland habitats. Sample sizes of traps are shown in parentheses.

\begin{tabular}{|c|c|c|c|c|c|c|c|c|}
\hline \multirow{2}{*}{ Species } & \multicolumn{4}{|c|}{ Mixedgrass Prairie $(n=3)$} & \multicolumn{4}{|c|}{ Coniferous Woodland $(n=2)$} \\
\hline & $\underset{(n=83)}{M S}$ & $\underset{\substack{\text { Sn=84) } \\
\text { SH }}}{ }$ & $\underset{(n=83)}{V}$ & $\underset{(n=84)}{P}$ & $\underset{(n=57)}{M S}$ & $\underset{(n=57)}{S H}$ & $\underset{(n=57)}{V}$ & $\underset{(n=57)}{P}$ \\
\hline Hispid Pocket Mouse & 0 & 0 & 0.02 & 0 & 0 & 0 & 0 & 0 \\
\hline Prairie Vole & 0.01 & 0 & 0 & 0 & 0.02 & 0 & 0 & 0 \\
\hline Deer Mouse & 0 & 0 & 0 & 0 & 0.11 & 0.05 & 0.05 & 0 \\
\hline Plains Harvest Mouse & 0.01 & 0 & 0 & 0 & 0 & 0 & 0 & 0 \\
\hline
\end{tabular}




\section{Pedro Primary Area}

Table D-11. Proportion of traplines each species was captured on within shrubland, coniferous woodland, and mixedgrass prairie habitats.

\begin{tabular}{|c|c|c|c|}
\hline Species & Shrubland $(\mathbf{n}=\mathbf{2})$ & Coniferous Woodland $(\mathbf{n}=\mathbf{2})$ & Mixedgrass Prairie $(\mathbf{n}=\mathbf{1})$ \\
\hline Prairie Vole & 0.5 & 0 & 1 \\
\hline Deer Mouse & 1 & 1 & 1 \\
\hline White-footed Mouse & 0.5 & 1 & 1 \\
\hline Plains Harvest Mouse & 0 & 0 & 1 \\
\hline
\end{tabular}

Table D-12. Proportion of museum special (MS), Sherman (SH), Victor (V), and pitfall (P) traps where small mammal species were detected within shrubland, coniferous woodland, and mixedgrass prairie habitats. Sample sizes of traps are shown in parentheses.

\begin{tabular}{|c|c|c|c|c|c|c|c|c|c|c|c|c|}
\hline \multirow[b]{2}{*}{ Species } & \multicolumn{4}{|c|}{ Shrubland $(n=2)$} & \multicolumn{4}{|c|}{ Coniferous woodland $(n=2)$} & \multicolumn{4}{|c|}{ Mixedgrass prairie $(n=1)$} \\
\hline & $\begin{array}{c}\text { MS } \\
(n=52) \\
\end{array}$ & $\begin{array}{c}\text { SH } \\
(n=53) \\
\end{array}$ & $\begin{array}{c}V \\
(n=53) \\
\end{array}$ & $\begin{array}{c}P \\
(n=60)\end{array}$ & $\begin{array}{c}\text { MS } \\
(n=53) \\
\end{array}$ & $\begin{array}{c}\mathrm{SH} \\
(n=59) \\
\end{array}$ & $\begin{array}{c}V \\
(n=53)\end{array}$ & $\begin{array}{c}P \\
(n=60) \\
\end{array}$ & $\begin{array}{c}\text { MS } \\
(n=23) \\
\end{array}$ & $\begin{array}{c}\text { SH } \\
(n=25)\end{array}$ & $\begin{array}{c}V \\
(n=22)\end{array}$ & $\underset{(n=30)}{P}$ \\
\hline Prairie Vole & 0.04 & 0 & 0 & 0 & 0 & 0 & 0 & 0 & 0 & 0 & 0.05 & 0 \\
\hline Deer Mouse & 0.08 & 0.02 & 0.04 & 0 & 0.08 & 0 & 0.04 & 0 & 0.04 & 0 & 0 & 0 \\
\hline $\begin{array}{c}\text { White-footed } \\
\text { Mouse }\end{array}$ & 0.02 & 0 & 0 & 0 & 0.11 & 0.02 & 0 & 0 & 0 & 0 & 0.05 & 0 \\
\hline $\begin{array}{c}\text { Plains Harvest } \\
\text { Mouse }\end{array}$ & 0 & 0 & 0 & 0 & 0 & 0 & 0 & 0 & 0.04 & 0 & 0 & 0 \\
\hline
\end{tabular}




\section{Mission Ridge Primary Area}

Table D-13. Proportion of traplines each species was captured on within mixedgrass prairie, barren, and shrubland habitats.

\begin{tabular}{|c|c|c|c|}
\hline Species & Mixedgrass Prairie $(\mathbf{n}=\mathbf{3})$ & Barren $(\mathbf{n}=\mathbf{1})$ & Shrubland $(\mathbf{n}=\mathbf{1})$ \\
\hline Least Shrew & 0.33 & 0 & 0 \\
\hline Deer Mouse & 0.67 & 1 & 0 \\
\hline White-footed Mouse & 0.33 & 1 & 1 \\
\hline
\end{tabular}

Table D-14. Proportion of museum special (MS), Sherman (SH), Victor (V), and pitfall (P) traps where small mammal species were detected within mixedgrass prairie, barren, and shrubland habitats. Sample sizes of traps are shown in parentheses.

\begin{tabular}{|c|c|c|c|c|c|c|c|c|c|c|c|c|}
\hline \multirow[b]{2}{*}{ Species } & \multicolumn{4}{|c|}{ Mixedgrass Prairie $(n=3)$} & \multicolumn{4}{|c|}{ Barren $(n=1)$} & \multicolumn{4}{|c|}{ Shrubland $(n=1)$} \\
\hline & $\underset{(n=75)}{M S}$ & $\underset{(n=85)}{S H}$ & $\underset{(n=85)}{V}$ & $\underset{(n=90)}{P}$ & $\underset{(n=24)}{M S}$ & $\underset{(n=30)}{S H}$ & $\underset{(n=30)}{V}$ & $\underset{(n=29)}{P}$ & $\underset{\substack{(n=29) \\
\text { MS }}}{M}$ & $\underset{\substack{\text { Sn=28) } \\
\text { SH }}}{ }$ & $\underset{(n=30)}{V}$ & $\underset{(n=30)}{P}$ \\
\hline Least Shrew & 0 & 0 & 0 & 0.01 & 0 & 0 & 0 & 0 & 0 & 0 & 0 & 0 \\
\hline Deer Mouse & 0.07 & 0 & 0.06 & 0 & 0.33 & 0.03 & 0.03 & 0 & 0 & 0 & 0 & 0 \\
\hline $\begin{array}{l}\text { White- } \\
\text { footed } \\
\text { Mouse }\end{array}$ & 0 & 0.01 & 0 & 0 & 0.04 & 0 & 0 & 0 & 0.03 & 0 & 0.03 & 0.03 \\
\hline
\end{tabular}




\section{Two Rivers Primary AREA}

Table D-15. Proportion of traplines each species was captured on within barren, coniferous woodland, and mixedgrass prairie habitats.

\begin{tabular}{|c|c|c|}
\hline Species & Barren $(\mathbf{n}=\mathbf{1})$ & Coniferous Woodland $(\mathbf{n}=\mathbf{2})$ \\
\hline Hispid Pocket Mouse & 0 & 0.5 \\
\hline Prairie Vole & 0 & 0.5 \\
\hline Deer Mouse & 1 & 1 \\
\hline White-footed Mouse & 1 & 0.5 \\
\hline Plains Harvest Mouse & 1 & 0 \\
\hline
\end{tabular}

Table D-16. Proportion of museum special (MS), Sherman (SH), Victor (V), and pitfall (P) traps where small mammal species were detected within barren, coniferous woodland, and mixedgrass prairie habitats. Sample sizes of traps are shown in parentheses.

\begin{tabular}{|c|c|c|c|c|c|c|c|c|c|c|c|c|}
\hline \multirow[b]{2}{*}{ Species } & \multicolumn{4}{|c|}{ Barren $(n=1)$} & \multicolumn{4}{|c|}{ Coniferous Woodland $(n=2)$} & \multicolumn{4}{|c|}{ Mixedgrass Prairie $(n=2)$} \\
\hline & $\begin{array}{c}\text { MS } \\
(n=25)\end{array}$ & $\begin{array}{c}\text { SH } \\
(n=29)\end{array}$ & $\underset{(n=27)}{V}$ & $\underset{(n=30)}{P}$ & $\underset{(n=56)}{M S}$ & $\begin{array}{c}\text { SH } \\
(n=60)\end{array}$ & $\begin{array}{c}V \\
(n=59)\end{array}$ & $\begin{array}{c}P \\
(n=60)\end{array}$ & $\underset{(n=55)}{M S}$ & $\begin{array}{c}\text { SH } \\
(n=58)\end{array}$ & $\underset{(n=55)}{V}$ & $\underset{(n=60)}{P}$ \\
\hline Hispid Pocket Mouse & 0 & 0 & 0 & 0 & 0 & 0.02 & 0 & 0 & 0 & 0.02 & 0.02 & 0 \\
\hline Prairie Vole & 0 & 0 & 0 & 0 & 0.02 & 0 & 0 & 0 & 0 & 0 & 0 & 0 \\
\hline Deer Mouse & 0.04 & 0 & 0.04 & 0 & 0.12 & 0 & 0.03 & 0 & 0.04 & 0 & 0.02 & 0 \\
\hline White-footed Mouse & 0.08 & 0 & 0 & 0 & 0.02 & 0 & 0 & 0 & 0 & 0 & 0 & 0 \\
\hline Plains Harvest Mouse & 0 & 0 & 0.04 & 0 & 0 & 0 & 0 & 0 & 0 & 0 & 0 & 0 \\
\hline
\end{tabular}




\section{APPENDIX E}

BASEline STATUS Indices OF DETECTED SPECIES: BAT SURVEYS 
Table E-1. Species definitively identified $(\mathrm{Y})$ in a given month with acoustic recordings made on long-term passive ultrasonic bat detectors in 3 primary survey areas.

\begin{tabular}{|c|c|c|c|c|c|c|c|c|c|c|c|c|}
\hline Detector & Species & $\begin{array}{c}\text { Oct, } \\
2013 \\
\end{array}$ & $\begin{array}{l}\text { Nov, } \\
2013 \\
\end{array}$ & $\begin{array}{l}\text { Dec, } \\
2013 \\
\end{array}$ & $\begin{array}{c}\text { Jan, } \\
2014 \\
\end{array}$ & $\begin{array}{l}\text { Feb, } \\
2014 \\
\end{array}$ & $\begin{array}{l}\text { Mar, } \\
2014 \\
\end{array}$ & $\begin{array}{l}\text { Apr, } \\
2014 \\
\end{array}$ & $\begin{array}{l}\text { May, } \\
2014 \\
\end{array}$ & $\begin{array}{c}\text { Jun, } \\
2014 \\
\end{array}$ & $\begin{array}{c}\text { Jul, } \\
2014 \\
\end{array}$ & $\begin{array}{l}\text { Aug, } \\
2014\end{array}$ \\
\hline \multirow{4}{*}{$\begin{array}{c}\text { Battle Creek, } \\
\text { Butte County Primary } \\
\text { Area, } \\
\text { Mixed Grass Prairie }\end{array}$} & Eastern Red Bat & & & & & & & & & & $Y$ & Y \\
\hline & Hoary Bat & & & & & & & & $\mathrm{Y}$ & $Y$ & $Y$ & $Y$ \\
\hline & Silver-haired Bat & & & & & & & & $Y$ & $Y$ & $Y$ & \\
\hline & Little Brown Myotis & & & & & & & & Y & $\mathrm{Y}$ & Y & $\mathrm{Y}$ \\
\hline \multirow{3}{*}{$\begin{array}{c}\text { South } \\
\text { Moreau River } \\
\text { Bridge, } \\
\text { Butte County Primary } \\
\text { Area, } \\
\text { Riparian Woodland }\end{array}$} & Silver-haired Bat & & & & & & & & $\mathrm{Y}$ & Y & $Y$ & $\mathrm{Y}$ \\
\hline & Western Small-footed Myotis & & & & & & & & $\mathrm{Y}$ & & $\mathrm{Y}$ & \\
\hline & Little Brown Myotis & & & & & & & & Y & Y & Y & $\mathrm{Y}$ \\
\hline \multirow{5}{*}{$\begin{array}{l}\text { Jug Creek, } \\
\text { Newell Primary Area, } \\
\text { Mixed Grass Prairie }\end{array}$} & Big Brown Bat & & & & & & & & & & $Y$ & \\
\hline & Hoary Bat & & & & & & & & & $Y$ & $Y$ & \\
\hline & Silver-haired Bat & & & & & & & & $Y$ & & $Y$ & \\
\hline & Western Small-footed Myotis & & & & & & & & & Y & $Y$ & \\
\hline & Little Brown Myotis & & & & & & & & $\mathrm{Y}$ & $\mathrm{Y}$ & $Y$ & $\mathrm{Y}$ \\
\hline \multirow{6}{*}{$\begin{array}{l}\text { Bismarck Bridge, } \\
\text { Newell Primary Area, } \\
\text { Riparian Woodland }\end{array}$} & Big Brown Bat & & & & & & & & $\mathrm{Y}$ & $Y$ & $Y$ & \\
\hline & Red bat & & & & & & & & & & $Y$ & \\
\hline & Hoary Bat & & & & & & & & & Y & $Y$ & \\
\hline & Silver-haired Bat & & & & & & & $\mathrm{Y}$ & $Y$ & $Y$ & $Y$ & $\mathrm{Y}$ \\
\hline & Western Small-footed Myotis & & & & & & & $Y$ & Y & $Y$ & $Y$ & $Y$ \\
\hline & Little Brown Myotis & & & & & & & & Y & Y & $\mathrm{Y}$ & $\mathrm{Y}$ \\
\hline \multirow{5}{*}{$\begin{array}{c}\text { Fort Meade } \\
\text { Reservoir, Fort } \\
\text { Meade Primary Area, } \\
\text { Mixed Grass Prairie }\end{array}$} & Big Brown Bat & $\mathrm{Y}$ & & & & & & $\mathrm{Y}$ & $Y$ & $\mathrm{Y}$ & Y & $Y$ \\
\hline & Eastern Red Bat & Y & & & & & & & & & $Y$ & $Y$ \\
\hline & Silver-haired Bat & & & & & & & $Y$ & Y & & & \\
\hline & Western Small-footed Myotis & & & & & & & $\mathrm{Y}$ & Y & Y & Y & Y \\
\hline & Little Brown Myotis & $\mathrm{Y}$ & & & & & & $\mathrm{Y}$ & Y & $\mathrm{Y}$ & $\mathrm{Y}$ & $\mathrm{Y}$ \\
\hline
\end{tabular}


Table E-2. Total bat passes, number of nights bats were detected, average and range of passes for nights bats were detected each month ultrasonic detectors were deployed.

\begin{tabular}{|c|c|c|c|c|c|}
\hline Detector Location & Date & $\begin{array}{l}\text { Total No. } \\
\text { Bat Passes }\end{array}$ & $\begin{array}{c}\text { No. Nights } \\
\text { with Detections }\end{array}$ & $\begin{array}{c}\text { Average Passes } \\
\text { Per Night (SE) }\end{array}$ & $\begin{array}{c}\text { Range of Passes } \\
\text { Per Night }\end{array}$ \\
\hline \multirow{11}{*}{$\begin{array}{c}\text { Battle Creek, Butte County } \\
\text { Primary Area, } \\
\text { Mixed Grass Prairie }\end{array}$} & Oct, 2013 & 0 & 0 & $0(0)$ & $0-0$ \\
\hline & Nov, 2013 & 0 & 0 & $0(0)$ & $0-0$ \\
\hline & Dec, 2013 & 0 & 0 & $0(0)$ & $0-0$ \\
\hline & Jan, 2014 & 0 & 0 & $0(0)$ & $0-0$ \\
\hline & Feb, 2014 & 0 & 0 & $0(0)$ & $0-0$ \\
\hline & Mar, 2014 & 0 & 0 & $0(0)$ & $0-0$ \\
\hline & Apr, 2014 & 0 & 0 & $0(0)$ & $0-0$ \\
\hline & May, 2014 & 691 & 23 & $30.0(55.4)$ & $1-264$ \\
\hline & Jun, 2014 & 361 & 26 & $13.9(15.4)$ & $1-62$ \\
\hline & Jul, 2014 & 421 & 30 & $14.0(10.4)$ & $1-54$ \\
\hline & Aug, 2014 & 73 & 8 & $9.1(6.3)$ & $5-23$ \\
\hline \multirow{11}{*}{$\begin{array}{c}\text { South Moreau Bridge, } \\
\text { Butte County Primary } \\
\text { Area, } \\
\text { Riparian Woodland }\end{array}$} & Oct, 2013 & 0 & 0 & $0(0)$ & $0-0$ \\
\hline & Nov, 2013 & 0 & 0 & $0(0)$ & $0-0$ \\
\hline & Dec, 2013 & 0 & 0 & $0(0)$ & $0-0$ \\
\hline & Jan, 2014 & 0 & 0 & $0(0)$ & $0-0$ \\
\hline & Feb, 2014 & 0 & 0 & $0(0)$ & $0-0$ \\
\hline & Mar, 2014 & 0 & 0 & $0(0)$ & $0-0$ \\
\hline & Apr, 2014 & 3 & 1 & $3.00\left(^{*}\right)$ & $3-3$ \\
\hline & May, 2014 & 91 & 22 & $4.1(2.5)$ & $1-11$ \\
\hline & Jun, 2014 & 67 & 22 & $3.1(3.3)$ & $1-16$ \\
\hline & Jul, 2014 & 238 & 27 & $8.8(6.7)$ & $2-36$ \\
\hline & Aug, 2014 & 64 & 8 & $8.0(4.7)$ & $3-17$ \\
\hline \multirow{11}{*}{$\begin{array}{l}\text { Jug Creek, } \\
\text { Newell Primary Area, } \\
\text { Mixed Grass Prairie }\end{array}$} & Oct, 2013 & 0 & 0 & $0(0)$ & $0-0$ \\
\hline & Nov, 2013 & 0 & 0 & $0(0)$ & $0-0$ \\
\hline & Dec, 2013 & 0 & 0 & $0(0)$ & $0-0$ \\
\hline & Jan, 2014 & 0 & 0 & $0(0)$ & $0-0$ \\
\hline & Feb, 2014 & 0 & 0 & $0(0)$ & $0-0$ \\
\hline & Mar, 2014 & 0 & 0 & $0(0)$ & $0-0$ \\
\hline & Apr, 2014 & 0 & 0 & $0(0)$ & $0-0$ \\
\hline & May, 2014 & 84 & 16 & $5.3(4.6)$ & $1-16$ \\
\hline & Jun, 2014 & 168 & 25 & $6.7(4.9)$ & $1-19$ \\
\hline & Jul, 2014 & 219 & 30 & $7.3(5.9)$ & $1-24$ \\
\hline & Aug, 2014 & 39 & 8 & $4.9(2.9)$ & $1-10$ \\
\hline
\end{tabular}




\begin{tabular}{|c|c|c|c|c|c|}
\hline Detector Location & Date & $\begin{array}{l}\text { Total No. } \\
\text { Bat Passes }\end{array}$ & $\begin{array}{c}\text { No. Nights } \\
\text { with Detections }\end{array}$ & $\begin{array}{c}\text { Average Passes } \\
\text { Per Night (SE) }\end{array}$ & $\begin{array}{c}\text { Range of Passes } \\
\text { Per Night } \\
\end{array}$ \\
\hline \multirow{11}{*}{$\begin{array}{l}\text { Bismarck Bridge, } \\
\text { Newell Primary Area, } \\
\text { Riparian Woodland }\end{array}$} & Oct, 2013 & 0 & 0 & $0(0)$ & $0-0$ \\
\hline & Nov, 2013 & 0 & 0 & $0(0)$ & $0-0$ \\
\hline & Dec, 2013 & 0 & 0 & $0(0)$ & $0-0$ \\
\hline & Jan, 2014 & 0 & 0 & $0(0)$ & $0-0$ \\
\hline & Feb, 2014 & 0 & 0 & $0(0)$ & $0-0$ \\
\hline & Mar, 2014 & 0 & 0 & $0(0)$ & $0-0$ \\
\hline & Apr, 2014 & 14 & 5 & $2.8(1.9)$ & $1-6$ \\
\hline & May, 2014 & 313 & 28 & $11.2(13.2)$ & $1-66$ \\
\hline & Jun, 2014 & 232 & 28 & $8.3(4.9)$ & $2-21$ \\
\hline & Jul, 2014 & 551 & 32 & $17.2(9.3)$ & $2-37$ \\
\hline & Aug, 2014 & 107 & 11 & $9.7(4.0)$ & $5-18$ \\
\hline \multirow{11}{*}{$\begin{array}{l}\text { Fort Meade Reservoir, } \\
\text { Fort Meade Primary Area, } \\
\text { Mixed Grass Prairie }\end{array}$} & Oct, 2013 & 119 & 6 & $19.8(25.7)$ & $1-69$ \\
\hline & Nov, 2013 & 18 & 5 & $3.6(3.7)$ & $1-10$ \\
\hline & Dec, 2013 & 0 & 0 & $0(0)$ & $0-0$ \\
\hline & Jan, 2014 & 0 & 0 & $0(0)$ & $0-0$ \\
\hline & Feb, 2014 & 0 & 0 & $0(0)$ & $0-0$ \\
\hline & Mar, 2014 & 0 & 0 & $0(0)$ & $0-0$ \\
\hline & Apr, 2014 & 1,173 & 14 & $83.8(121.6)$ & $1-458$ \\
\hline & May, 2014 & 5,616 & 29 & $193.7(188.1)$ & $1-698$ \\
\hline & Jun, 2014 & 4,542 & 28 & $162.2(210.5)$ & $1-933$ \\
\hline & Jul, 2014 & 6,132 & 31 & $197.8(263.5)$ & $17-1282$ \\
\hline & Aug, 2014 & 541 & 11 & $49.2(77.4)$ & $1-276$ \\
\hline
\end{tabular}




\section{Appendix F}

\section{Baseline Status Indices of Detected SpeCies: Amphibian Calling Surveys}




\section{Butte County Primary Area}

Table F-1. Proportion of points where species were detected and average estimated number of individuals at points where they were detected. Species denoted with + are BLM Sensitive.

\begin{tabular}{|c|c|c|}
\hline Species & $\begin{array}{c}\text { Proportion of Points } \\
\text { Detected (N) }\end{array}$ & $\begin{array}{c}\text { Average Number of Individuals } \\
\text { Estimated at Points Detected (SE) }\end{array}$ \\
\hline Boreal Chorus Frog & $0.87(34)$ & $12.0(17.8)$ \\
\hline Northern Leopard Frog & $0.23(9)$ & $1.3(0.5)$ \\
\hline Plains Spadefoot + & $0.15(6)$ & $3.0(1.3)$ \\
\hline
\end{tabular}

\section{Newell Primary Area}

Table F-2. Proportion of points where species were detected and average estimated number of individuals at points where they were detected.

\begin{tabular}{|c|c|c|}
\hline Species & $\begin{array}{c}\text { Proportion of Points } \\
\text { Detected (N) }\end{array}$ & $\begin{array}{c}\text { Average Number of Individuals } \\
\text { Estimated at Points Detected (SE) }\end{array}$ \\
\hline Boreal Chorus Frog & $1.00(12)$ & $9.7(8.5)$ \\
\hline
\end{tabular}

\section{Fort Meade Primary ARea}

Table F-3. Proportion of points where species were detected and average estimated number of individuals at points where they were detected.

\begin{tabular}{|c|c|c|}
\hline Species & $\begin{array}{c}\text { Proportion of Points } \\
\text { Detected (N) }\end{array}$ & $\begin{array}{c}\text { Average Number of Individuals } \\
\text { Estimated at Points Detected (SE) }\end{array}$ \\
\hline Boreal Chorus Frog & $0.44(8)$ & $4.1(2.3)$ \\
\hline Northern Leopard Frog & $0.06(1)$ & $2\left(^{*}\right)$ \\
\hline
\end{tabular}

\section{Mission Ridge Primary Area}

Table F-4. Proportion of points where species were detected and average estimated number of individuals at points where they were detected.

\begin{tabular}{|c|c|c|}
\hline Species & $\begin{array}{c}\text { Proportion of Points } \\
\text { Detected (N) }\end{array}$ & $\begin{array}{c}\text { Average Number of Individuals } \\
\text { Estimated at Points Detected (SE) }\end{array}$ \\
\hline Woodhouse's Toad & $0.17(5)$ & $2.6(1.1)$ \\
\hline Boreal Chorus Frog & $0.69(20)$ & $5.0(4.5)$ \\
\hline
\end{tabular}




\section{TWO RIVERS Primary AReA}

Table F-5. Proportion of points where species were detected and average estimated number of individuals at points where they were detected. Species denoted with + are BLM Sensitive.

\begin{tabular}{|c|c|c|}
\hline Species & $\begin{array}{c}\text { Proportion of Points } \\
\text { Detected (N) }\end{array}$ & $\begin{array}{c}\text { Average Number of Individuals } \\
\text { Estimated at Points Detected (SE) }\end{array}$ \\
\hline Boreal Chorus Frog & $0.88(22)$ & $3.1(2.1)$ \\
\hline Great Plains Toad + & $0.76(19)$ & $4.5(2.6)$ \\
\hline Woodhouse's Toad & $0.32(8)$ & $2.8(2.7)$ \\
\hline Plains Spadefoot + & $0.48(12)$ & $7.3(5.0)$ \\
\hline
\end{tabular}

